\title{
1 Bewertung der Rechtslage ab 25.05.2018
}

\subsection{Einleitung}

Das Bundesministerium für Wirtschaft und Energie (BMWi) fördert das Projekt „Smart Analysis - Health Research Access“ (SAHRA) der AOK Nordost. Über die webbasierte SAHRA-Plattform sollen Sozialdaten der AOK Nordost kombiniert, referenziert und validiert und schließlich zugunsten von Wissenschaft, Versorgung und Industrie verwendet werden.

Die Technologie- und Methodenplattform für die vernetzte medizinische Forschung e.V. (TMF) hat im Rahmen des SAHRA-Projekts im Mai 2017 den Auftrag für ein Rechtsgutachten zur Nutzung von Sozial- und Gesundheitsdaten ausgeschrieben.

Das vorliegende Gutachten wurde ursprünglich zum 23.09.2017 eingereicht und beachtete die bis dahin bekannten Reformvorhaben. Punktuelle Änderungen redaktioneller Art wurden bis 18.12.2017 vorgenommen. Vor dem Hintergrund anhaltender datenschutzrechtlicher Reformen, insbesondere in den für dieses Gutachten maßgeblichen fachspezifischen Bereichen der Gesetzlichen Krankenversicherung, wie sie im Sozialgesetzbuch Fünftes Buch (SGB V) verankert sind, wurde nunmehr eine Aktualisierung bis zum 19.10.2018 vorgenommen. Trotz der zwischenzeitlichen Geltungserlangung der Datenschutz-Grundverordnung (DSGVO) und des Inkrafttretens des neuen BDSG

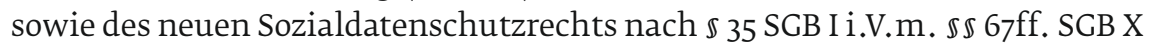


sind die Reformen des SGB V zur Anpassung an die DSGVO bei Redaktionsschluss nicht abgeschlossen.

Die Autoren bedauern diesen Umstand, hoffen mit der aktuellen Bearbeitung jedoch den Entwicklungen angemessen Rechnung tragen zu können.

\subsubsection{Problemstellung und Zielsetzung}

Forschungsprojekte, Qualitätssicherungsanalysen, Planungsvorhaben und sonstige statistische Auswertungen, die auf der Verarbeitung personenbezogener Daten basieren, unterliegen den Beschränkungen des Datenschutzrechts. Handelt es sich bei den Datensätzen um solche, die sensible Daten, wie etwa Gesundheitsdaten oder Sozialdaten, umfassen, so bestehen besonders hohe Hürden. Während das Datenschutzrecht durch ein Verbot mit Zulässigkeitsvorbehalt und das Gebot der Zweckbindung die Verwendung personenbezogener Daten auf ein unerlässliches Maß reduzieren möchte, ist Forschung zunehmend von der Verfügbarkeit und Validität umfassender Datenbestände abhängig. Diesem Bedürfnis liegt unter anderem eine Veränderung methodischer Forschungsansätze zugrunde. So erfordern insbesondere explorative Datenanalysen im Bereich des sog. Big Data große Datenvorräte, die nicht erst nach Formulierung einer konkreten, im Rahmen eines Forschungsprojekts zu untersuchenden These zielgerichtet erhoben werden können. Aber auch im Bereich der klassischen Forschungsvorhaben wirkt das Datenschutzrecht als ein Hemmnis. Dieses besteht aber nicht im Wesentlichen in den Anforderungen zum Schutz der betroffenen Personen, sondern in der Inkompatibilität paralleler Datenschutzgesetze auf Bundes- und Landesebene und der sektoralen Begrenzung bereichsspezifischen Datenschutzrechts.

Mit der Datenschutz-Grundverordnung (DSGVO) ${ }^{1}$ war eine weitgehende Vollharmonisierung des Datenschutzrechts in der Europäischen Union beabsichtigt. Im Verordnungstext sowie in den Erwägungsgründen ist ein forschungsfreundlicheres Grundverständnis angelegt. Gleichzeitig beinhaltet die DSGVO Öffnungsklauseln, die sowohl hinsichtlich ihrer Zahl als auch ihres Umfangs weitgehende Regelungen durch die Mitgliedsstaaten ermöglichen oder erfordern, ohne dabei von den wesentlichen Grundsätzen der DSGVO abzuweichen. Im Spannungsfeld der Intention der DSGVO und dem sich abzeichnenden Bestreben des Bundesgesetzgebers, das bestehende Datenschutzrecht weitgehend aufrechtzuerhalten, stellt sich die Frage nach einer rechtssicheren Ausgestaltung vernetzter Forschung sowie nach Qualitätssicherungs- und Planungsmechanismen, wie sie mit der SAHRA-Plattform beabsichtigt sind.

1 Hierzu siehe Kap. 1.1.1. 


\subsubsection{Gang der Untersuchung}

Die TMF hat als Auftraggeber des vorliegenden Gutachtens ein Pflichtenheft erstellt, das konkrete Fragen enthält. Das vorliegende Gutachten umfasst die Ausarbeitung der im Pflichtenheft als „Los 1“ zusammengefassten Kapitel I-III. „Los 2“, das Kapitel IV des Pflichtenhefts umfasst, wird anderweitig bearbeitet.

Die Fragen zu Los 1 beziehen sich weitgehend auf drei Sachverhaltsgestaltungen (Szenarien), anhand derer die künftige Rechtslage nach bisher bekanntem Gesetzgebungsstand zur zukünftigen Rechtslage (Kapitel I) und dem aktuellen rechtlichen Status quo (Kapitel II) untersucht werden sollen. Im Anschluss sind Reformüberlegungen anzustellen (Kapitel III). Diese Gliederung weicht insofern von den Vorgaben des Pflichtenheftes ab, als die künftige Rechtslage nunmehr in Kapitel I und nicht in Kapitel II dargestellt werden soll, was der besseren Lesbarkeit geschuldet ist. Dieses Vorgehen wurde im Rahmen der Vorgespräche zur Vergabe des Gutachtens mit dem Auftraggeber abgestimmt.

\subsubsection{Stand der Gesetzgebung}

Das Gutachten wird zu einem Zeitpunkt anhaltender Reformen des Datenschutzrechts erstellt. Auftraggeber und Gutachter haben sich darauf geeinigt, die reformierte Rechtslage bis zum 19. Oktober $2018 \mathrm{zu}$ berücksichtigen, soweit mindestens öffentlich einsehbare Gesetzentwürfe als parlamentarische Drucksachen vorliegen. Der Stand der Reformen gestaltet sich wie folgt:

\subsubsection{Bereits geltende Rechtslage}

Die „Verordnung (EU) 2016/679 des Europäischen Parlaments und des Rates vom 27. April 2016 zum Schutz natürlicher Personen bei der Verarbeitung personenbezogener Daten, zum freien Datenverkehr und zur Aufhebung der Richtlinie 95/46/EG (Datenschutz-Grundverordnung)“2 (DSGVO) wurde am 04. Mai 2016 im Amtsblatt der Europäischen Union veröffentlicht und trat am 24. Mai 2016 in Kraft. Sie ist seit dem 25. Mai 2018 in allen Mitgliedsstaaten unmittelbar anwendbar.

Der deutsche Bundesgesetzgeber ist in der Zwischenzeit tätig geworden und hat die Anpassung sowohl des allgemeinen Datenschutzrechts in Form des Bundesdatenschutzgesetzes (BDSG) als auch des allgemeinen Teils des bereichsspezifischen Datenschutzrechts des Sozialgesetzbuchs, der als Sozialdaten-

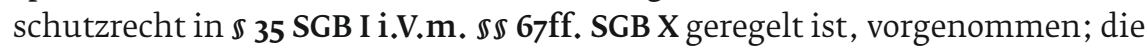

2 Abl. L 119 vom 04.05.2016, 1; korrigiert durch Abl. L 314 vom 22.11.2016, 72. 
Änderungen sind gleichzeitig mit der Wirkungserlangung der DSGVO zum 25.05.2018 in Kraft getreten. ${ }^{3}$

Das BDSG a.F. ist mit dem „Gesetz zur Anpassung des Datenschutzrechts an die Verordnung (EU) 2016/679 und zur Umsetzung der Richtlinie (EU) 2016/680 (Datenschutz-Anpassungs- und -Umsetzungsgesetz EU - DSAnpUG-EU)“ vom 30. Juni $2017^{4}$ von einem neuen Bundesdatenschutzgesetz (BDSG) abgelöst worden (vgl. Art. 8 DSAnpUG-EU).

In vergleichbarer Weise ist mit dem „Gesetz zur Änderung des Bundesversorgungsgesetzes und anderer Vorschriften" vom 17. Juli $2017^{5}$ auch das sog. Sozialdatenschutzrecht $\mathbb{} 35$ SGB I a.F. i.V.m. $\$ \$ \$ 67 f$. SGB X a.F.) umfassend neu geregelt (vgl. Art. 19 und Art. 23 des Gesetzes) worden.

Parallel zu den vorgenannten Gesetzgebungsvorhaben zur Anpassung der Rechtslage an die DSGVO hat der Bundestag die Änderung der Schweigepflichten von Berufsgeheimnisträgern beschlossen. Mit dem „Gesetz zur Neuregelung des Schutzes von Geheimnissen bei der Mitwirkung Dritter an der Berufsausübung schweigepflichtiger Personen" vom 30.10.2017 ${ }^{6}$ wurde die Schweigepflicht aus $\mathbb{S} 203$ StGB dahingehend geändert, dass die Beteiligung von "sonstigen Mitwirkenden“ ermöglicht wird, was insbesondere im Bereich des Outsourcings und der Auftrags(daten)verarbeitung neue Handlungsoptionen der Berufsgeheimnisträger schaffen soll.

\subsubsection{Ausstehende Änderungen und Gesetzesentwürfe}

Änderungen weiterer bereichsspezifischer Datenschutzgesetze stehen zum Zeitpunkt der Erstellung dieses Gutachtens noch aus. Es besteht daher eine rechtliche Ungewissheit, die mit Blick auf das vorliegende Gutachten insbesondere die datenschutzrechtlichen Regelungen des SGB V betrifft.

\subsection{Zweites Datenschutz-Anpassungs- und Umsetzungsgesetz EU - 2. DSAnpUG-EU (Entwurf)}

Der Gesetzentwurf eines Zweiten Gesetzes zur Anpassung des Datenschutzrechts an die Verordnung (EU) 2016/679 und zur Umsetzung der Richtlinie (EU) 2016/680 (Zweites Datenschutz-Anpassungs- und Umsetzungsgesetz EU 2. DSAnpUG-EU ${ }^{7}$ enthält umfangreiche Änderungen diverser datenschutz-

3 Weiterhin wurde das allgemeine Landesdatenschutzrecht in den jeweiligen Landesdatenschutzgesetzen aller 16 Bundesländer an die DS-GVO angepasst. Das bereichsspezifische Landesdatenschutzrecht ist noch nicht vollständig überarbeitet. Landesdatenschutzrecht kann für die vorliegende Begutachtung aber außer Betracht bleiben.

4 BGBI. I 2017, 2097.

5 BGBL. I 2017, 2541.

6 BGBL. I 2017, 3618.

7 Gesetzentwurf der Bundesregierung Entwurf eines Zweiten Gesetzes zur Anpassung des Datenschutzrechts an die Verordnung (EU) 2016/679 und zur Umsetzung der Richtlinie (EU) 2016/680 (Zweites Datenschutz-Anpassungs- und Umsetzungsgesetz EU - 2. DSAnpUG-EU), BR-Drs. 430/18; vgl. BT-Drs. 19/4674. 
rechtlicher Normen, insbesondere solcher des BDSG, SGB I, SGB V und SGB X. Zum Zeitpunkt der Begutachtung lag der Kabinettsentwurf dem Bundesrat zur Beratung vor und die Ausschüsse hatten bereits ihre Empfehlungen ${ }^{8}$ abgegeben. Der diesbezügliche Beschluss des Bundesrates vom 19.10.20189 sieht keine Änderung des Entwurfs für die hier in Betracht kommenden Regelungsbereiche des BDSG oder der bereichsspezifischen Normen des SCB I, SGB V oder SGB X vor. Der Gesetzentwurf enthält im Wesentlichen sprachliche Anpassungen an die DSGVO. Die Begriffe „erheben“, „nutzen“ und „verarbeiten“ sollen regelmäßig durch den umfassenderen Begriff „,verarbeiten“ im Sinne der DSGVO ersetzt werden, ohne damit aber inhaltliche Änderungen zu veranlassen. Weiterhin sollen etwa bisher streng formulierte Schriftformerfordernisse für datenschutzrechtliche Einwilligungen im SGB V dahingehend gelockert werden, dass auch elektronische Einwilligungen zulässig sein sollen.

Während $₫ 67 \mathrm{~b}$ Abs. 2 SGB X bislang lediglich eine Soll-Vorschrift beinhaltet, wonach zu Nachweiszwecken nach Art. 7 DSGVO eine Einwilligung in die Verarbeitung von personenbezogenen Daten schriftlich oder elektronisch erfolgen „soll“, dies gleichwohl keine Wirksamkeitsvoraussetzung für eine Einwilligung ist, wird nun vorgeschlagen, durch einen neuen Satz 2 zu regeln, dass Einwilligungen zur Verarbeitung von genetischen, biometrischen oder Gesundheitsdaten (oder Betriebs- und Geschäftsgeheimnissen) zwingend schriftlich oder elektronisch zu erfolgen haben, soweit nicht wegen besonderer Umstände eine andere Form angemessen ist. Aus der Begründung des Gesetzentwurfs ergibt sich, dass es sich hierbei nicht nur um ein Nachweiserfordernis, sondern um ein Wirksamkeitserfordernis handelt: Unter Bezugnahme auf die Öffnungsklausel des Art. 9 Abs. 4 DSGVO wird nur für die vorgenannten Datenkategorien eine strengere Form verlangt, was dazu dienen soll, das Schutzniveau der Altregelung des $\$ 67 \mathrm{~b}$ Abs. 2 S. 2 SGB X a.F. im zulässigen Umfang zu erhalten. ${ }^{10}$ Gleichzeitig sieht der Gesetzentwurf vor, dass in $\$ 67 \mathrm{~b}$ Abs. 3 SGB X für Verarbeitungen zu Forschungszwecken geregelt werden soll, dass ein besonderer Umstand, unter dem ein Abweichen von der vorgenannten Formvorgabe möglich ist, dann vorliege, wenn durch die Einholung einer schriftlichen oder elektronischen Einwilligung der Forschungszweck erheblich beeinträchtigt würde. Die Gründe hierfür sollen schriftlich festgehalten werden. Die vorgeschlagenen Regelungen sind zwar europarechtlich zulässig, sie führen jedoch zu zusätzlichen Hindernissen und ggf. zu weiterem Begründungsaufwand. Auf weitere Änderungen im SGB X wird an den entsprechenden Stellen des Gutachtens hingewiesen, sofern sich hieraus nach bisheriger Einschätzung relevante Änderungen ergeben können.

Die daneben bestehenden Änderungen des SGB V spielen für das vorliegende Gutachten nach bisherigem Erkenntnisstand hingegen keine Rolle. Sie

\footnotetext{
8 BR-Drs. 430/1/18.

9 BR-Drs. 430/18(B), Beschlussdrucksache.

10 BT-Drs. 19/4674, S. 400.
} 
betreffen etwa die Streichung der Pflicht zur Löschung von personenbezogenen Daten nach Zweckerreichung gem. \$284 Abs. 1 S. 4 und Abs. 4 S. 4 SGB V, die jedoch wegen der unmittelbaren Geltung von Art. 5 Abs. 1 lit. e) und Art. 17 Abs. 1 lit. a) DSGVO nicht zu einer inhaltlichen Änderung führt. ${ }^{11}$ Zentrale forschungsrelevante Vorschriften (wie $\$ 287$ SGB V) sollen nicht geändert werden.

\subsection{Terminservice- und Versorgungsgesetz - TSVG (Entwurf)}

Der Entwurf eines Gesetzes für schnellere Termine und bessere Versorgung (Terminservice- und Versorgungsgesetz - TSVG) hat am 26.09.2018 das Kabinett passiert und liegt zum Zeitpunkt des Redaktionsschlusses entsprechend nur als Kabinettsentwurf vor. Der Gesetzentwurf enthält umfangreiche Änderungen des SGB V, aber keine Anpassungen an die DSGVO. Mit dem Gesetzesvorhaben soll etwa das Mindestsprechstundenangebot der niedergelassenen Ärzte erhöht werden. Außerdem sollen Krankenkassen nach dem Gesetzentwurf ihren Versicherten spätestens ab 2021 eine elektronische Patientenakte (ePA) zur Verfügung stellen - ein Zugriff auf die in der elektronischen Akte gespeicherten medizinischen Daten soll auch mittels Smartphone oder Tablet möglich sein. Außerdem sind Änderungen im Bereich der Datenverarbeitungsbefugnisse der Krankenkassen nach $\$ 284$ SGB V und zum Umgang mit Daten zu Qualitätssicherungszwecken nach $\mathbb{2} 299$ SGB V vorgesehen. Diese Änderungen haben nach Ansicht der Gutachter aber keinen Einfluss auf die vorliegenden Fragestellungen.

\subsection{Darstellung des Rechtsrahmens}

\subsubsection{Unionsrechtlicher Rechtsrahmen}

Der unionsrechtliche Rahmen des Datenschutzrechts gliedert sich zunächst in das Primärrecht, das das Verfassungsrecht der Europäischen Union bildet, und das Sekundärrecht, das der europäische Gesetzgeber aufgrund der ihm im Sinne einer begrenzten Einzelermächtigung übertragenen Gesetzgebungskompetenz selbst erlassen kann.

\subsubsection{Primärrechtlicher Rechtsrahmen (EUV, AEUV, GRCh)}

Zum europäischen Primärrecht zählen der Vertrag über die Europäische Union (EUV) und der Vertrag über die Arbeitsweise der Europäischen Union (AEUV). ${ }^{12}$

11 BT-Drs. 19/4674, S. $140,370$.

12 Vertrag über die Europäische Union (konsolidierte Fassung) und Vertrag über die Arbeitsweise der Europäischen Union (konsolidierte Fassung), ABI. C 326 vom 26.10.2012, 1. 
Weiterhin ist hiervon die Charta der Grundrechte der Europäischen Union (GRCh) umfasst (Art. 6 Abs. 1 Hs. 2 EUV). ${ }^{13}$

Art. 16 Abs. 2 AEUV beinhaltet eine unionsrechtliche Gesetzgebungskompetenz für das Datenschutzrecht. Aus Art. 16 Abs. 1 AEUV sowie aus Art. 8 GRCh folgen zudem subjektiv-öffentliche Rechte in Form eines Datenschutzgrundrechts. Maßgeblich ist nach herrschender Meinung allein Art. 8 GRCh. ${ }^{14}$ Unmittelbar aus Art. 8 Abs. 2 S. 1 GRCh ergeben sich bereits die wesentlichen Erfordernisse der Verarbeitung nach „Treu und Glauben“, der Zweckbindung und eines Legitimationstatbestandes, welcher entweder in einer Einwilligung oder einer sonstigen gesetzlich geregelten legitimen Grundlage bestehen kann (Verbot mit Zulässigkeitsvorbehalt). ${ }^{15}$

\subsubsection{Sekundärrechtlicher Rechtsrahmen (DSGV0)}

Sekundärrechtlich war das Datenschutzrecht bisher durch die „Richtlinie zum Schutz natürlicher Personen bei der Verarbeitung personenbezogener Daten und zum freien Datenverkehr"16 (RL 95/46/EG), die Datenschutzrichtlinie (DSRL), umgesetzt. Die Handlungsform der Richtlinie war zwar hinsichtlich des zu erreichenden Ziels verbindlich, überließ jedoch den innerstaatlichen Stellen die Wahl der Form und der Mittel bei der Umsetzung, vgl. Art. 288 Abs. 3 AEUV (ex-Art. 189 Abs. 3 EGV) ${ }^{17}$.

Nunmehr hat sich der europäische Gesetzgeber für eine andere Handlungsform entschieden. Das neue sekundärrechtliche Datenschutzrecht ist als Verordnung im Sinne des Art. 288 Abs. 2 AEUV ausgestaltet. Die Verordnung (EU) 2016/679 „zum Schutz natürlicher Personen bei der Verarbeitung personenbezogener Daten, zum freien Datenverkehr und zur Aufhebung der Richtlinie 95/46/EG (Datenschutz-Grundverordnung)“ (DSGVO) hat allgemeine Geltung, ist in allen ihren Teilen verbindlich und gilt in jedem Mitgliedsstaat unmittelbar. Als „Grund“-Verordnung regelt sie die wesentlichen Teile selbst, enthält allerdings eine Vielzahl von Öffnungsklauseln, die den Mitgliedsstaaten Gesetzgebungsspielräume eröffnen. Die DSGVO präsentiert sich dadurch als „Hybrid“ zwischen Verordnung und Richtlinie. ${ }^{18}$

Die Öffnungsklauseln beschränken sich dabei nicht darauf, den Mitgliedsstaaten lediglich die optionale Möglichkeit des gesetzgeberischen Tätigwerdens einzuräumen (fakultative Öffnungsklausel), sondern sehen mitunter

\footnotetext{
13 Ruffert, in: Callies/Ruffert, EUV/AEUV, 5. Aufl., 2016, Art. 1 AEUV Rn. 8.

14 Kingreen, in: Callies/Ruffert, EUV/AEUV, 5. Aufl., 2016, Art. 8 GRCh Rn. 3 (m.w.N.).

15 Ein solches Verbot mit Zulässigkeitsvorbehalt (häufig als „Verbot mit Erlaubnisvorbehalt“ bezeichnet) ergibt sich für öffentliche Stellen bereits aus dem allgemeinen Parlamentsvorbehalt.

16 ABI. L 281 vom 23.11.1995, S. 31.

17 Vertrag zur Gründung der Europäischen Gemeinschaft, Abl. C 224 vom 31.08.1992, 1.

18 Kühling/Martini et al., Die DSGVO und das nationale Recht, 2016, S. 1.
} 
Regelungsbefehle vor, mithin Verpflichtungen zum gesetzgeberischen Tätigwerden der Mitgliedsstaaten (obligatorische Öffnungsklausel). ${ }^{19}$

Im hier relevanten öffentlichen Bereich bestehen weitreichende Öffnungsklauseln, die den Erlass von Rechtsgrundlagen und die Regelung von Einzelheiten der Verarbeitung ermöglichen (vgl. Art. 6 Abs. 1 UAbs. 1 lit. c), e), Abs. 2, 3 DSGVO). ${ }^{20}$ Sofern auch besondere Kategorien personenbezogener Daten (insb. Gesundheitsdaten) betroffen sind, sieht Art. 9 Abs. 2, 3, 4 DSGVO besondere Öffnungsklauseln vor. Neben der Möglichkeit, die Einwilligung in die Verarbeitung im jeweiligen Mitgliedsstaat auszuschließen (Art. 9 Abs. 2 lit. a) DSGVO), betreffen die Öffnungsklauseln speziell in Art. 9 Abs. 2 lit. b), g), h) und i), Abs. 4 DSGVO das öffentliche Gesundheitswesen sowie Art. 9 Abs. 2 lit. j) i.V.m. Art. 89 DSGVO insb. den Bereich der wissenschaftlichen Forschung und damit den der medizinischen Forschung.

Im Ergebnis besteht bei einer Zusammenschau der Öffnungsklauseln ein umfangreicher Gestaltungsspielraum für die Mitgliedsstaaten im Bereich der öffentlichen Gesundheit. ${ }^{21}$ Eine strikte Trennung zwischen den Öffnungsklauseln des Art. 6 und des Art. 9 DSGVO ist nicht zwingend erforderlich, da sie ggf. auch gemeinsam zur Anwendung gebracht werden können, sofern sie nicht im Widerspruch zueinanderstehen. Ein durch eine Öffnungsklausel den Mitgliedsstaaten überantworteter Regelungsbereich wird nicht dadurch negiert, dass er Schnittmengen mit einer weiteren Öffnungsklausel hat. Vielmehr kann dies den Regelungsspielraum erweitern.

\subsubsection{Deutsches Datenschutzrecht}

Das deutsche Datenschutzrecht ist zunächst vom Fehlen einer Gesetzgebungskompetenz des Bundes geprägt. Die Regelung des Datenschutzrechts stellt sich vielmehr als Kompetenz kraft Sachzusammenhang oder Annexkompetenz dar, sodass je nach zu Regeln der Hauptmaterie entweder der Bund im Falle des Eingreifens einer ausschließlichen oder konkurrierenden Gesetzgebungskompetenz (Art. 71ff. GG) oder aber die Länder als grundsätzlich Gesetzgebungsbefugte (Art. 7o GC) tätig werden können. Das führt zu einem Nebeneinander sowohl von allgemeinem Datenschutzrecht als auch von bereichsspezifischem Datenschutzrecht auf Bundes- wie auf Landesebene. Hinzu kommen Regelungsbefugnisse der Kirchen in ihren eigenen Angelegenheiten. ${ }^{22}$

19 Kühling/Martini et al., Die DSGVO und das nationale Recht, 2016, S. gff.

20 Kühling/Martini et al., Die DSGVO und das nationale Recht, 2016, S. $13 f$.

21 Vgl. Weichert, in: Kühling/Buchner, DS-GVO, 2017, Art. 9 Rn. 170.

22 Hierzu vertiefend: Kircher, Der Schutz personenbezogener Gesundheitsdaten im Gesundheitswesen, 2016, S. $81 \mathrm{ff}$. 


\subsubsection{Allgemeines Datenschutzrecht (BDSG; ggf. Landesdatenschutzgesetze)}

Als allgemeines Datenschutzrecht galt bisher auf Bundesebene das Bundesdatenschutzgesetz (BDSG a.F.). Alle 16 Bundesländer haben zusätzlich Landesdatenschutzgesetze erlassen. Das BDSG a.F. fand grundsätzliche Anwendung auf öffentliche Stellen des Bundes sowie auf nicht-öffentliche Stellen. Die Landesdatenschutzgesetze fanden Anwendung für die öffentlichen Stellen der Länder.

Mit dem BDSG hat der Bundesgesetzgeber sich dafür entschieden, die bisherige Struktur soweit wie möglich beizubehalten. Zwar folgen die wesentlichen Grundsätze nunmehr unmittelbar aus der DSGVO, das BDSG bildet jedoch auch in Zukunft das allgemeine Datenschutzrecht auf nationaler Ebene.

Gleiches gilt für die Landesdatenschutzgesetze; die allgemeinen Landesdatenschutzgesetze wurden sämtlich an die DSGVO angepasst.

\subsubsection{Bereichsspezifisches Datenschutzrecht (Sozialdatenschutzrecht nach SGB I/X-neu, sozialrechtliches Fachrecht, sonstige bereichsspezifische Regelungen)}

Mit dem Sozialdatenschutzrecht nach $\mathbb{} 35$ SGB I a.F. i.V.m. $\mathbb{S} \mathbb{S} 67 f f$. SGB X a.F. hatte der Bund aufgrund seiner Gesetzgebungskompetenz aus Art. 74 Abs. 1 Nr. 12 i.V.m. Art. 72 Abs. 1 GG eine weitere datenschutzrechtliche „Vollregelung“ ${ }_{23}$ als allgemeinen Teil des bereichsspezifischen Datenschutzrechts geschaffen, die durch die speziellen Regelungen der sozialrechtlichen Fachbücher, wie etwa den $\mathbb{S} 284 \mathrm{ff}$. SGB V, ergänzt wurde. $\$ 35$ SGB I a.F. enthielt als „Grundnorm des Sozialdatenschutzes " 24 den Anspruch, dass Sozialdaten nicht unbefugt von Leistungsträgern und den weiteren abschließend in $₫ 35$ Abs. 1 SGB I a.F. genannten Stellen verarbeitet werden. Gemäß $\mathbb{3 5}$ Abs. 2 SGB I a.F. richtete sich die Erhebung, Verarbeitung und Nutzung von Sozialdaten nach dem zweiten Kapitel des SGB X a.F. Diese $\$ \$ S$ 67f. SGB X a.F. normierten von Begriffsdefinitionen, über Zulässigkeitstatbestände, Betroffenenrechte und Sanktionen alle wesentlichen datenschutzrechtlichen Inhalte und bedurften keines Rückgriffs auf das BDSG a.F. als allgemeinen Teil des deutschen Datenschutzrechts.

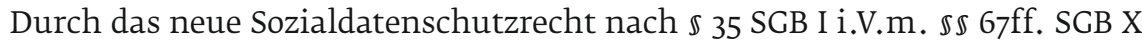
wird auch im Sozialgesetzbuch die bisherige Struktur im Wesentlichen beibehalten. Auch hier gilt, dass der Bundesgesetzgeber lediglich solche Änderungen vorgenommen hat, die sich nach seiner Einschätzung als notwendig erwiesen, um dem unmittelbaren Geltungsanspruch der DSGVO gerecht zu werden. Die gewachsenen Strukturen des Sozialdatenschutzrechts sollten aber möglichst weitgehend beibehalten werden. Eine Zusammenschau des neuen

23 Dix, in: Simitis (Hrsg.), BDSG, 8. Aufl., 2014, §1 Rn. 160.

24 Binne, NZS 1995, 97 (100); BT-Dr. 8/4022, S. 80. 
Sozialdatenschutzrechts zeigt, dass nahezu alle Normen geändert wurden, wobei ein großer Teil der Änderungen redaktioneller Art ist und beispielsweise eine Anpassung an die neuen Begriffsdefinitionen des Art. 4 DSGVO, beinhaltet. Unmittelbar aus den Änderungen des SCB, aber auch mittelbar aus der geänderten sekundärrechtlichen Gewährleistung der DSGVO, folgen insgesamt inhaltliche Änderungen, wobei die wesentlichen Strukturen innerhalb des SGB aufrechterhalten bleiben.

Ausdrücklich normiert hat der Gesetzgeber in $₫ 35$ Abs. 2 Satz 1 SGB I nunmehr, dass die „Bücher des Sozialgesetzbuchs“ die „Verarbeitung von Sozialdaten abschließend“" regeln, soweit nicht die DSGVO unmittelbar gilt. Damit wird klargestellt, dass die DSGVO zwar unmittelbar Geltung erlangt, dort aber wo der deutsche Bundesgesetzgeber auf eine Öffnungsklausel zurückgreifen kann und dies auch getan hat, die Regelungen des Sozialdatenschutzrechts abschließend gelten sollen. Weiterhin wird durch die Klarstellung Bezug auf die Regelung des $\mathbb{1} 1$ Abs. 2 S. 2 BDSG genommen, wonach ein Rückgriff auf das BDSG als allgemeines deutsches Bundesdatenschutzrecht nur möglich ist, sofern eine spezielle bundesrechtliche Vorschrift nicht abschließend regelt. Es gilt also neben der DSGVO ausschließlich das Sozialgesetzbuch für die Verarbeitung von Sozialdaten. Der Anspruch des SCB, die Sozialversicherung als Vollregelung umfassend zu regeln, wird nur insoweit eingeschränkt, als der europarechtliche Anwendungsvorrang der DSGVO dies erfordert.

Aus der Gesetzesbegründung zur Änderung des SGB I und SGB X wird deutlich, dass die Bundesregierung die neuen Bestimmungen sämtlich auf die Öffnungsklauseln der Art. 6 Abs. 2 und 3 sowie Art. 9 Abs. 2 DSGVO stützt. ${ }^{25}$ Art. 6 Abs. 2 DSGVO eröffnet den Mitgliedsstaaten den größten Spielraum. Er ist dann einschlägig, wenn die Verarbeitung gemäß Art. 6 Abs. 1 lit. e) DSGVO für die Wahrnehmung einer Aufgabe erforderlich ist, die im öffentlichen Interesse liegt, oder die in Ausübung öffentlicher Gewalt erfolgt. Die Öffnungsklausel ermöglicht damit den Erlass oder die Beibehaltung von Regelungen im Bereich der sozialen Sicherheit sowohl für Behörden, für die dies eine Aufgabe in Ausübung öffentlicher Gewalt darstellt, sowie für Private, die eine im öffentlichen Interesse liegende Aufgabe wahrnehmen. Werden besondere Kategorien personenbezogener Daten - beispielsweise Gesundheitsdaten - verarbeitet, sind die Öffnungsklauseln des Art. 9 Abs. 2 lit. b), g), h) und i) DSGVO zu beachten. Ein vollständiges systematisches Regelungsregime im Bereich der sozialen Sicherheit ist daher auf Art. 6 Abs. 2 DSGVO zu stützen. Diese Öffnungsklausel hat sich insbesondere Deutschland im Rat erkämpft, um eigene gewachsene Systeme beibehalten zu können, die den spezifischen hiesigen Eigenheiten im öffentlichen Bereich Rechnung tragen. Damit bleibt es bei einem geschlossenen System des Sozialdatenschutzes. ${ }^{26}$

25 Vgl. BT-Drs. 18/12611, S. $104 f$.

26 So auch Hoidn, in: Roßnagel (Hrsg.), Das neue Datenschutzrecht, 2018, S. $294 f$. 
Regelungen der DSGVO, die unmittelbar Anwendung finden, sind beispielsweise die Grundprinzipien der Datenverarbeitung ${ }^{27}$ oder die grundlegenden Prinzipien der Datensicherheit sowie die Pflicht des Verantwortlichen zur Datenschutz-Folgenabschätzung nach Art. 35 DSGVO, die „Selbstregulierung“ mittels Verhaltensregeln nach Art. 40, 41 DSGVO und Zertifizierungen nach Art. 42, 43 DSGVO. ${ }^{28}$

An dieser Stelle sei allerdings darauf hingewiesen, dass mit dem neu einge-

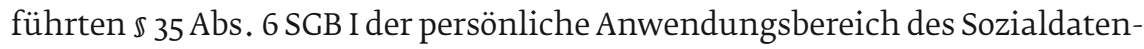
schutzrechts im Vergleich zur alten Fassung so verstanden werden könnte, dass er auf solche Stellen ausgeweitet wurde, die nicht bereits in $\mathbb{} 35 \mathrm{Abs} .1$ SGB I genannt sind. So heißt es in $₫ 35$ Abs. 6 Satz 1 SGB I:

„Die Absätze 1 bis 5 finden neben den in Absatz 1 genannten Stellen auch Anwendung auf solche Verantwortliche oder deren Auftragsverarbeiter,

1. die Sozialdaten im Inland verarbeiten, sofern die Verarbeitung nicht im Rahmen einer Niederlassung in einem anderen Mitgliedstaat der Europäischen Union oder in einem anderen Vertragsstaat des Abkommens über den Europäischen Wirtschaftsraum erfolgt, oder

2. die Sozialdaten im Rahmen der Tätigkeiten einer inländischen Niederlassung verarbeiten." [Hervorhebung nicht im Original]

In Zusammenschau mit der Begriffsdefinition des $₫ 67$ Abs. 2 S. 1 SGB X könnte sich ein Zirkelschluss ergeben, der nur schwer auflösbar scheint. $\$ 67$ Abs. 2 S. 1 SGB X lautet:

„Sozialdaten sind personenbezogene Daten (Artikel 4 Nummer 1 der Verordnung [EU] 2016/679), die von einer in $\mathbb{S} 35$ des Ersten Buches genannten Stelle im Hinblick auf ihre Aufgaben nach diesem Gesetzbuch verarbeitet werden." [Hervorhebung nicht im Original]

Entweder liefe $\mathbb{3} 35$ Abs. 6 SGB I leer, weil die nicht explizit in Absatz 1 des $\mathbb{} 35$ SGB I genannten Stellen gemäß der Definition des $₫ 67$ Abs. 2 SGB X keine Sozialdaten verarbeiten können, oder aber Absatz 6 ist geeignet, die Anwendbarkeit des Sozialdatenschutzrechts nahezu unbegrenzt auszuweiten. Letzteres wäre etwa dann der Fall, wenn man nunmehr davon ausgehen müsste, dass eine Transformation eines personenbezogenen Datums in ein Sozialdatum zukünftig irreversibel ist. Jedes Sozialdatum würde bei einer weiteren Verarbeitung - und nicht nur bei einer zweckgebundenen Weiterverarbeitung nach einer Übermittlung durch eine in $₫ 35$ Abs. 1 SGB I genannte Stelle - immer dem Sozialdatenschutzrecht unterliegen, womit die Bestimmung des

27 Hierzu s. Kap. 1.3.

$28 \mathrm{Vgl}$. Bieresborn, NZS 2017, 887 (889). 
einschlägigen Datenschutzrechts nicht mehr an der verantwortlichen Stelle ansetzen würde, sondern am Charakter des Datums. Das wäre hochproblematisch, da einem Datum seine Herkunft in aller Regel nicht anzusehen ist. Aus den Gesetzgebungsunterlagen ergibt sich lediglich ein Hinweis auf $₫ 78$ Abs. 1 S. 2 (sic!) SGB X ${ }^{29}$, wonach Dritte, denen Sozialdaten übermittelt wurden, diese Daten in gleichem Umfang geheim zu halten haben, wie die in $₫ 35$ SGB I genannten Stellen. Die Gesetzesbegründung verweist also auf einen falschen Satz, der seinerseits dem Wortlaut nach nur die Rechtsfolge einer Geheimhaltungspflicht beinhaltet, inhaltlich aber wohl die Anwendbarkeit des Sozialdatenschutzrechts bedeuten könnte. Insgesamt muss hier von einer nachlässigen Gesetzgebungsarbeit ausgegangen werden, die einer Ermittlung der legislativen Absicht entgegensteht. Da sich aus den Gesetzgebungsmaterialien jedenfalls keine Absichten entnehmen lassen, den Geltungsbereich des Sozialdatenschutzrechts auszuweiten, ist eine restriktive Auslegung des $\mathbb{3} 35 \mathrm{Abs} .6$ SCB I geboten. ${ }^{30}$

\subsubsection{Verhältnis zu Geheimnis- und Schweigepflichten (Insb. § 203 StGB)}

Nach bisheriger Rechtslage war das Verhältnis zwischen Datenschutzrecht und Geheimnis- oder Schweigepflichten in $\mathbb{1} 1 \mathrm{Abs}$. 3 S. 2 BDSG a.F. geregelt. Demnach blieb die Verpflichtung zur Wahrung gesetzlicher Geheimnispflichten oder von Berufs- oder besonderen Amtsgeheimnissen, die nicht auf gesetzlichen Vorschriften beruhen, unberührt. Mit anderen Worten waren Datenschutzrecht und Geheimnispflichten parallel anzuwenden (sog. Zwei-Schranken-Prinzip). Durchbrechungen dieses Prinzips bestanden nur in wenigen Ausnahmefällen.

Das BDSG enthält ebenfalls eine ausdrückliche Regelung zum Verhältnis der beiden Rechtsinstrumente zueinander. Gemäß $₫ 1$ Abs. 2 S. 2 BDSG bleibt auch in Zukunft die Wahrung gesetzlicher Geheimhaltungspflichten oder von Berufs- oder besonderen Amtsgeheimnissen, die nicht auf gesetzlichen Vorschriften beruhen, unberührt. Zum Zweck der Klarstellung wurde eine gleichlautende Regelung nunmehr auch für das Sozialdatenschutzrecht in $\mathbb{} 35$ Abs. za SGB I aufgenommen. Hierbei ist darauf hinzuweisen, dass das Sozialgeheimnis nach $₫ 35 \mathrm{Abs}$. 1 S. 1 SGB I selbst keine solche Geheimhaltungspflicht beinhaltet, sondern lediglich einen Anspruch auf Einhaltung datenschutzrechtlicher Anforderungen formuliert. Die Bezeichnung als Sozialgeheimnis ist daher irreführend und lediglich noch historisch zu erklären, ${ }^{31}$ wenngleich eine Notwendigkeit zur Aufrechterhaltung dieses Instruments für die Anpassungsgesetzgebung an die DSGVO nicht mehr besteht.

29 Gemeint war wohl $\$ 78$ Abs. 1 S. 3 SGB X oder $\$ 78$ Abs. 1 S. 2 SGB X a.F.

30 Siehe hierzu auch Kapitel 1.5.4.1.2.

31 Mrozynski, SGB I, 5. Aufl., 2014, §35 Rn. 9; Kircher, Der Schutz personenbezogener Gesundheitsdaten im Gesundheitswesen, 2016, S. 46f., $113 f$. 
Über die bloße parallele Geltung hinaus, sieht das Sozialdatenschutzecht mit-

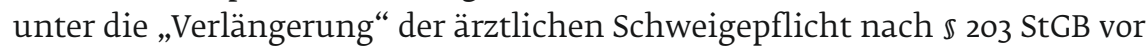
und bestimmt so, dass auch Empfänger der Daten diese entsprechend geheim halten müssen (vgl. $\mathbb{} 76$ Abs. 1 SGB X ${ }^{32}$ ). Das hat zur Folge, dass zusätzlich zur datenschutzrechtlichen Zulässigkeit eine Offenbarungsbefugnis nach den Maßstäben des Strafrechts erforderlich ist, wenn ein Empfänger die Daten seinerseits weitergeben möchte. ${ }^{33}$ Diese Anforderung perpetuiert sich aus der sog. Erstübermittlung mit jeder weiteren Übermittlung. ${ }^{34}$

Am Vorrang der Geheimnispflichten ändert auch die Reform des $\mathbb{2} 203$ StGB nichts. Durch die nunmehr bestehende Möglichkeit, „sonstige Mitwirkende“ befugt an einem Geheimnis teilhaben lassen zu können, ist zwar die Möglichkeit geschaffen worden, externe Dienstleister beispielsweise im Rahmen eines Auftragsverarbeitungsverhältnisses in eine berufliche Tätigkeit einzubinden. Auf eine zwischenzeitlich diskutierte Lösung, datenschutzrechtliche Vorschriften als Befugnisnorm im Sinne des $\mathbb{2 0 3}$ StCB auszugestalten, wurde jedoch verzichtet. Gleichzeitig knüpft auch der neue $\$ 203$ StGB nicht an datenschutzrechtliche Kategorien oder Instrumente, wie die Auftragsverarbeitung, an. Die strafrechtlichen Voraussetzungen für die Einbeziehung Mitwirkender ergeben sich vielmehr unmittelbar aus dem Wortlaut des nunmehr geltenden $\$ 203$ StGB.

Im Bereich der Heilberufe ist zu beachten, dass auch die Reform der strafrechtlichen Schweigepflicht die jeweiligen Berufsordnungen unberührt lässt. Auch wenn also eine Offenbarung gegenüber „sonstigen Mitwirkenden“ strafrechtlich befugt sein mag, so kann sie (derzeit) dennoch berufsrechtswidrig sein.

\subsection{Wesentliche Prinzipien des Datenschutzrechts}

Die DSGVO benennt in Art. 5 vorab neun allgemeine Grundsätze, die für die Verarbeitung personenbezogener Daten maßgeblich sind. Diese Grundsätze werden durch die konkreten Vorschriften der DSGVO sowie einzelner bereichsspezifischer Datenschutzregelungen konkretisiert und durch weitere nicht explizit genannte Grundsätze ergänzt. Diese nachfolgend kurz skizzierten Crundsätze sind bei jeder Form der Verarbeitung personenbezogener Daten zu beachten und bei der Auslegung datenschutzrechtlicher Gesetze heranzuziehen.

\subsubsection{Rechtmäßigkeit der Verarbeitung und Verbot mit Zulässigkeitsvorbehalt}

Crundlegendes Prinzip der Verarbeitung personenbezogener Daten ist, dass diese auf rechtmäßige Weise erfolgen muss (Art. 5 Abs. 1 lit. a) DSGVO). Hand

32 Gleiches galt bereits nach alter Rechtslage gem. \$76 Abs. 1 SGB X.

33 Vgl. Diering/Seidel, in: Diering/Timme, SGB X, 4. Aufl., 2016, \$ 76 Rn. 7.

34 Hierzu Meier, Der rechtliche Schutz patientenbezogener Gesundheitsdaten, 2003, S. $235 \mathrm{ff}$. 
in Hand mit diesem Prinzip geht das auch im Rahmen der DSGVO geltende Prinzip des Verbots mit Erlaubnisvorbehalt. Entsprechend diesen Prinzipien ist eine Verarbeitung personenbezogener Daten grundsätzlich verboten, es sei denn die Verarbeitung ist entsprechend Art. 6 DSGVO sowie Art. 9 DSGVO durch einen gesetzlichen Erlaubnistatbestand oder die Einwilligung des Betroffenen legitimiert.

\subsubsection{Zweckbindung}

Einen weiteren wesentlichen Pfeiler der Verarbeitung personenbezogener Daten bildet der Zweckbindungsgrundsatz. Gemäß Art. 5 Abs. 1 lit. b) DSGVO dürfen Daten nur für festgelegte, eindeutige und legitime Zwecke erhoben und verarbeitet werden. Demnach hat bereits im Vorfeld der Datenerhebung eine Zweckfestlegung zu erfolgen. Dieser festgelegte Zweck begrenzt die Datenverarbeitungsmöglichkeiten und bestimmt die Einzelheiten der Verarbeitung. Dabei ist zu beachten, dass der ursprünglich festgelegte Verarbeitungszweck eine Verarbeitung zu anderen Zwecken nicht generell ausschließt. Vielmehr kann eine Datenverarbeitung auch zu anderen Zwecken erfolgen. Nach einer Ansicht erfordert dies, dass die Weiterverarbeitung zum Sekundärzweck auf eine gesonderte Rechtsgrundlage gestützt werden kann und zudem mit dem ursprünglichen Zweck der Datenerhebung vereinbar ist. ${ }^{35} \mathrm{Nach}$ anderer Ansicht bedarf es bei Vereinbarkeit des Sekundärzwecks mit dem Primärzweck keiner eigenen Rechtsgrundlage. ${ }^{36}$ Letzterer Ansicht ist zuzustimmen. Zum einen stimmt sie mit der Aussage des Erwägungsgrundes 50 überein, nach dessen Satz 2 für eine zweckvereinbare Weiterverwendung keine „andere gesonderte" Rechtsgrundlage erforderlich ist. Zum anderen würde die Zweckvereinbarkeitsprüfung weitgehend leerlaufen, wenn es dennoch einer neuen Rechtsgrundlage bedürfte. ${ }^{37}$

Bei der Frage der Vereinbarkeit mit dem ursprünglichen Zweck spielen unterschiedliche Faktoren eine Rolle, die im Rahmen einer wertenden Betrachtung einzubeziehen sind. Neben Nähe und Kontext des neuen zum ursprünglichen Zweck sind auch die Art der Daten, die Folgen der Weiterverarbeitung sowie ein Wechsel des Verantwortlichen von entscheidender Bedeutung. ${ }^{38}$ Insbesondere im Bereich der Verarbeitung besonderer Kategorien personenbezogener Daten sind der Zweckvereinbarung enge Grenzen gesetzt. 39 Für den Bereich der wissenschaftlichen Forschung stellt Art. 5 DSGVO jedoch die Vermu-

35 Heberlein, in: Ehmann/Selmayr, DS-GV0, 2017, Art. 5 Rn. 19f.; Herbst, in: Kühling/Buchner, DS-GV0, 2017, Art. 5 Rn. $24 f f$.

36 Frenzel, in: Paal/Pauly, DS-GV0, 2017, Art. 5 Rn. 30f.; vgl. Erw.Gr. 50 Abs. 1 S. 2.

$37 \mathrm{Vgl.} \mathrm{Nebel,} \mathrm{Erlaubnis} \mathrm{zur} \mathrm{Datenverarbeitung,} \mathrm{in:} \mathrm{Roßnagel} \mathrm{(Hrsg.),} \mathrm{Europäische} \mathrm{Datenschutz-Grundverordnung,}$ 2017, § 3 Rn. 97.

38 Herbst, in: Kühling/Buchner, DS-GV0, 2017, Art. 5 Rn. 44.

39 Herbst, in: Kühling/Buchner, DS-GVO, 2017, Art. 5 Rn. 44. 
tung auf, dass dieser neue Zweck mit dem ursprünglichen Zweck vereinbar ist, sofern die Verarbeitung entsprechend Art. 89 Abs. 1 DSGVO erfolgt.

Für Sozialdaten greift \$ 67c SGB X den Zweckbindungsgrundsatz auf und regelt, dass Sozialdaten grundsätzlich nur zur Erfüllung der gesetzlich übertragenen Aufgaben verarbeitet werden dürfen, sofern die Daten hierfür erforderlich sind und für diesen Zweck erhoben wurden. Eine Datenverarbeitung zu anderen Zwecken ist nur in engen Grenzen zulässig (vgl. z.B. $\$ 284$ Abs. 3 SGB V, $\mathbb{5} 67$ c Abs. 2 Nr. 2 SGB X, $\mathbb{S} 75$ SGB X). Dem Bereich der wissenschaftlichen Forschung kommt in diesem Zusammenhang eine gewisse Privilegierung zu (vgl. Erw.Gr. 33).

\subsubsection{Treu und Glauben}

Das Gebot der Verarbeitung personenbezogener Daten entsprechend Treu und Glauben ist der Übersetzung der DSGVO ins Deutsche geschuldet und ist weniger im Sinne des in der deutschen Rechtsprechung und Literatur zu $\$ 242$ BGB entwickelten Verständnisses, sondern vielmehr im Sinne der Gewährleistung einer „fairen“ Verarbeitung zu verstehen. ${ }^{40}$ Im Übrigen entspricht dies jedoch der Terminologie des Art. 8 Abs. 2 GRCh. Über diesen Auffangtatbestand soll in jedem Fall ein Ausgleich der widerstreitenden Interessen der betroffenen Personen und der Verantwortlichen sowie die Herstellung des Kräftegleichgewichts möglich sein ${ }^{41}$. Dementsprechend sind in den unterschiedlichen Datenverarbeitungsschritten, etwa bei der Information des Betroffenen, im Rahmen der Bewertung der Freiwilligkeit der Einwilligung oder von Art und Umfang der Datenverarbeitung sowie der Abwägung widerstreitender Interessen oder der Festlegung von Verhaltensregeln nach Art. 40 Abs. 2 lit. a) DSGVO die vernünftigen Erwartungen der betroffenen Person zugrunde zu legen (Erw.Gr. 47 S. 1, Erw.Gr. 50 S. 6).

\subsubsection{Datenminimierung (Datenvermeidung und Datensparsamkeit)}

Neben der Zweckbindung gibt Art. 5 Abs. 1 lit. c) DSGVO vor, dass die Verarbeitung entsprechend der Zweckbestimmung auf ein notwendiges Maß zu beschränken ist. Dementsprechend darf eine Verarbeitung personenbezogener Daten nicht erfolgen, wenn der Zweck auch auf andere Weise, etwa auf Crundlage anonymisierter Daten, zu erreichen ist ${ }^{42}$. Dieser Grundsatz wird in Art. 25 DSGVO konkretisiert, gemäß dem geeignete technische und organisatorische Maßnahmen - etwa privacy by design und privacy by default - zu ergreifen sind. Für den Bereich der wissenschaftlichen Forschung stellt sich in diesem

40 Heberlein, in: Ehmann/Selmayr, DS-GV0, 2017, Art. 5 Rn. 9; Plath, in: BDSG/DSGV0, 2017, \5 Rn. 9.

41 Herbst, in: Kühling/Buchner, DS-GV0, 2017, Art. 5 Rn. 17.

42 Heberlein, in: Ehmann/Selmayr, DS-GVO, 2017, Art. 5 Rn. 22. 
Zusammenhang folglich regelmäßig die Frage, inwiefern die Verarbeitung personenbezogener Daten tatsächlich erforderlich ist oder ob nicht vielmehr auch die Arbeit mit anonymisierten oder zumindest pseudonymisierten Daten ausreicht.

\subsubsection{Transparenz}

Gemäß Art. 5 Abs. 1 lit. a) DSGVO sollen personenbezogene Daten in einer für den Betroffenen transparenten, nachvollziehbaren Weise verarbeitet werden. Dies setzt voraus, dass der Betroffene umfassend über die Art und den Umfang der Verarbeitung, die Identität des Verantwortlichen und die Zwecke der Verarbeitung sowie über Risiken, Vorschriften, Garantien und seine Rechte im Zusammenhang mit der Verarbeitung seiner personenbezogenen Daten unterrichtet wird (Erw.Gr. 39). Darüber hinaus sind diese Informationen dem Betroffenen in klarer, leicht verständlicher Form und ohne Zugangshindernisse zur Verfügung zu stellen. Dieser allgemeine Transparenzgrundsatz wird durch die spezifischen geregelten Informationspflichten der Verantwortlichen (Art. 12-14 DSGVO) sowie Auskunftsrechte der Betroffenen (Art. 15 DSGVO) konkretisiert und durch technische Maßnahmen wie etwa datenschutzfreundliche Einstellungen und Zertifizierungsverfahren flankiert.

\subsubsection{Richtigkeit der Daten}

Was im BDSG a.F. über die Rechte auf Löschung und Berichtigung personenbezogener Datenverbürgt war, hat nunmehr in Art. 5 Abs. 1 lit. e) DSGVO eine ausdrückliche Regelung erfahren. Danach müssen personenbezogene Daten sachlich richtig sein und auf dem neuesten Stand gehalten werden. Die Durchsetzung dieses Grundsatzes ist auch nach der DSGVO durch Ansprüche auf Berichtigung und Löschung gewährleistet.

\subsubsection{Speicherbegrenzung}

Art. 5 Abs. 1 lit. e) DSGVO bestimmt, dass die Speicherdauer auf den für die Zweckerfüllung zwingend erforderlichen Zeitraum zu begrenzen ist. Zwar bedeutet das nicht, dass die Speicherfrist kalendermäßig bestimmt sein muss. Jedoch muss sich die Dauer der Speicherung zumindest anhand eines Ereignisses oder einer Bedingung bestimmen lassen. Für Daten, die für Zwecke der wissenschaftlichen Forschung verarbeitet werden, gilt jedoch wiederum eine Ausnahme von dieser Regelung, sofern Garantien vorliegen, über die sichergestellt wird, dass geeignete technische und organisatorische Maßnahmen bestehen, mit denen die Einhaltung der Datenverarbeitungsgrundsätze gewährleistet und die Rechte und Interessen der Betroffenen geschützt werden. 


\subsubsection{Integrität und Vertraulichkeit}

Um die Integrität und Vertraulichkeit personenbezogener Daten zu gewährleisten, verpflichtet Art. 5 Abs. 1 lit. f) DSGVO den Verantwortlichen, geeignete technische und organisatorische Maßnahmen zu treffen, die die Daten vor einem unautorisierten Zugriff - etwa durch Zugangs- und Zugriffskontrollen - oder dem Verlust - durch entsprechende Sicherungsmaßnahmen - bewahren.

\subsubsection{Rechenschaftspflicht}

Nach Art. 5 Abs. 2 DSGVO wird dem Verantwortlichen die Gesamtverantwortung für eine rechtmäßige Datenverarbeitung und die Einhaltung der übrigen Datenverarbeitungsgrundsätze auferlegt und darüber hinaus eine diesbezügliche Nachweispflicht geregelt. Im Rahmen dieser Gesamtverantwortung sind im Vorfeld der Datenverarbeitung im Einzelfall Risikoanalysen und Datenschutz-Folgenabschätzungen (Art. 35 DSGVO) vorzunehmen und ein Verarbeitungsverzeichnis (Art. 30 DSGVO) anzulegen. Während der Verarbeitung sind die Daten durch technische und organisatorische Maßnahmen (Art. 32 DSGVO) - wie etwa Zugangs- und Zugriffskontrollen sowie Back-ups - im Rahmen eines umfassenden Datenschutzmanagements zu sichern. Zudem sind Reaktions- und Meldesysteme für Fälle von Datenschutzverletzungen (Art. 33, 34 DSGVO) vorzusehen. All diese Maßnahmen sind in regelmäßigen Abständen auf Aktualität und Suffizienz zu überprüfen und zum Zwecke des Nachweises (Art. 5 Abs. 2 DSGVO) zu dokumentieren. Dabei erstrecken sich die Verantwortung für die Verarbeitung und die Nachweispflicht nicht nur auf die Verarbeitung durch den Verantwortlichen selbst, sondern umfassen auch die Verarbeitung durch Auftragsverarbeiter.

\subsubsection{Direkterhebung}

Der noch in $\$ 4$ Abs. 2 BDSG a.F. ausdrücklich normierte Grundsatz der Direkterhebung ist in der DSGVO nicht mehr explizit geregelt. Auch wenn es an einer ausdrücklichen Regelung fehlt, bleibt dieser Grundsatz im Rahmen der Datenverarbeitungsgrundsätze zur Transparenz und der Datenminimierung sowie der Erforderlichkeit der Datenverarbeitung auch im Anwendungsbereich der DSGVO von Bedeutung ${ }^{43}$. Für den Bereich des Sozialdatenschutzrechts wurde der Grundsatz der Direkterhebung auch nach der Reform ausdrücklich in $\$ 67 a$ Abs. 2 S. 1 SGB X aufgenommen, sodass Sozialdaten nur in den gesetzlich geregelten Ausnahmefällen ( $\$ 67 a$ Abs. 2 S. 2 SCB X) bei Dritten erhoben werden dürfen.

43 Bäcker, in: Kühling/Buchner, DS-GV0, 2017, Art. 13 Rn. 3. 


\subsubsection{Erforderlichkeit}

Auch wenn dieser Grundsatz nicht ausdrücklich in Art. 5 DSGVO genannt ist, wird er an mehreren Stellen des Gesetzes verwendet (etwa in Art. 6 Abs. 1 lit. b)-f) DSGVO). In Verbindung mit anderen Datenverarbeitungsgrundsätzen - etwa der Datenminimierung und der Speicherbegrenzung - stellt das Erforderlichkeitsprinzip eine wesentliche Säule des Datenschutzes dar. Die Datenverarbeitung muss sich in Art und Umfang auf ein Maß beschränken, das zur Erreichung des Zwecks notwendig und ausreichend ist (siehe hierzu die Ausführungen im Gutachtenteil von Roßnagel im vorliegenden Band.). ${ }^{44}$

\subsection{Beurteilung von Szenario 1}

Im Folgenden wird auf die einzelnen Szenarien eingegangen. Entsprechend der Ausführungen in Kapitel 1.1.1 wird die Rechtslage nach der DSGVO sowie dem bislang bekannten nationalen Datenschutzrecht beurteilt. In Ermangelung einer Neufassung der datenschutzrechtlichen Normen der fachspezifischen Sozialgesetzbücher (insb. SGB V) kann hier nur auf die geltende Rechtslage abgestellt werden.

\subsubsection{Darstellung des Szenarios}

Die Datennutzung geschieht im Rahmen eines Kooperationsprojekts einer gesetzlichen Krankenkasse („Leistungsträger“ i.S.d. Sozialversicherungsrechts) mit einer externen Einrichtung. Die Fragestellungen für die Datenauswertung werden gemeinsam entwickelt oder von der externen Einrichtung vorgegeben. Die Sozialdaten bleiben in der Einrichtung der gesetzlichen Krankenkasse und werden hier ausgewertet. Lediglich anonyme Auswertungsergebnisse werden an den externen Partner übermittelt, es verlassen keine Sozialdaten die Einrichtung der gesetzlichen Krankenversicherung.

\subsubsection{Rechtfertigungsbedürftige Datenverarbeitungsvorgänge}

Vorab ist festzuhalten, dass die Weitergabe der anonymen Auswertungsergebnisse durch den Leistungsträger nicht rechtfertigungsbedürftig ist, denn aus Sicht des Empfängers werden anonyme Daten weitergegeben. Unter der Prämisse, dass die Daten durch den Leistungsträger im Rahmen des $\mathbb{} 284$ Abs. 1 S. 1 SGB V erhoben und gespeichert wurden, sind die Erhebung und Speicherung der Daten insofern gerechtfertigt und nicht Gegenstand nachfolgender Prüfung.

44 Buchner/Petri, in: Kühling/Buchner, DS-GV0, 2017, Art. 6 Rn. 15. 
Datenschutzrechtsrelevante Datenumgänge, deren Zulässigkeit nachfolgend zu prüfen ist, sind somit im Szenario 1 allein die Auswertungen der Daten durch den Leistungsträger zu Zwecken der wissenschaftlichen Forschung, der Planung im Sozialleistungsbereich und der Qualitätssicherung.

\subsubsection{Zulässigkeit der Datenumgänge durch den Leistungsträger (hinsichtlich des jeweiligen Zwecks: Wissenschaftliche Forschung, Planung im Sozialleistungsbereich oder Qualitätssicherung)}

Zulässig ist die Auswertung der Sozialdaten nur im Falle eines einschlägigen Erlaubnistatbestandes und bei Vorliegen aller seiner Voraussetzungen. Im Rahmen der nachfolgenden Prüfung ist angesichts der zahlreichen Verortungen von datenschutzrechtlichen Vorschriften zunächst der Prüfungsmaßstab unter Berücksichtigung des einschlägigen Datenschutzregimes zu klären (s. Kap. 1.4.3.1). Sodann ist zu untersuchen, ob bzw. welche Erlaubnistatbestände des einschlägigen Datenschutzregimes greifen (s. Kap. 1.4.3.2 und Kap. $1.4 \cdot 3 \cdot 3)$.

\subsubsection{Prüfungsmaßstab/Identifikation des einschlägigen Datenschutzregimes}

Die Frage, welches Datenschutzregime im Einzelnen Anwendung findet, richtet sich danach, welche Stelle für die Verarbeitung personenbezogener Daten verantwortlich ist.

Im vorliegenden Szenario werden personenbezogene Daten ausschließlich von einer Krankenkasse verarbeitet. Krankenkassen unterliegen als Leistungsträ-

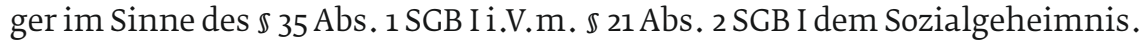
Sie sind somit an das umfassend regelnde Sozialdatenschutzrecht nach $\mathbb{} 35$

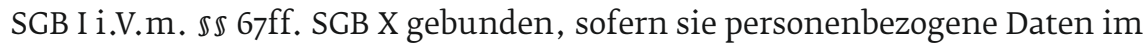
Hinblick auf ihre Aufgaben nach dem Sozialgesetzbuch (SCB), also Sozialdaten im Sinne des $\mathbb{5} 67$ Abs. 2 S. 1 SGB X, verarbeiten. Weiterhin müssen zusätzlich

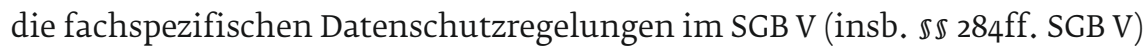
beachtet werden. Nach dem Wortlaut des $\mathbb{3} 35$ Abs. 2 S. 1 SGB I regeln die Vorschriften des Sozialdatenschutzrechts und der übrigen Bücher des SGB die Verarbeitung von Sozialdaten abschließend, soweit nicht die DSGVO unmittelbar gilt. Das heißt, dass also zunächst die unmittelbar geltende DSGVO Anwendung findet; auf nationaler Ebene sind dann die datenschutzrechtlichen Regelungen im SGB zu beachten. Ein Rückgriff auf das allgemeine Bundesdatenschutzrecht im BDSG kommt hingegen nicht in Betracht (vgl. $\mathbb{1}$ Abs. 2 S. 2 BDSG). Das BDSG kann nur dann noch Anwendung finden, wenn eine Norm des SGB ausdrücklich auf das BDSG verweist. ${ }^{5}$ Der Anspruch des SGB, die Verarbeitung von Sozialdaten abschließend zu regeln, gilt aber auch

45 Vgl. BT-Drs. 18/12611, S. 96. 
im Verhältnis zur DSGVO, wenn im Sozialdatenschutz aufgrund einer Öffnungsklausel der DSGVO eine spezifische Regelung getroffen wird. ${ }^{46}$

Lediglich im Falle der Einwilligung und in Fällen der Verarbeitung zum Schutz lebenswichtiger Interessen kann sich die Rechtfertigung - ohne Rückgriff auf das Sozialgesetzbuch - unmittelbar aus Art. 9 Abs. 2 lit a) und c) DSGVO ergeben. Da zur Regelung der allgemeinen Einwilligung keine Öffnungsklausel mehr besteht und hinsichtlich der Öffnungsklausel zum Ausschluss der Einwilligung in die Verarbeitung sensibler Daten kein Gebrauch gemacht wurde, erscheint es folgerichtig, dass in Zukunft - abweichend von den grundsätzlichen Erwägungen des BSC ${ }^{47}$ - auch hinsichtlich der Möglichkeit der Einholung von Einwilligungserklärungen auf allgemeines Datenschutzrecht in Form der DSGVO zurückgegriffen werden kann. ${ }^{48}$

\subsection{Verbot mit Zulässigkeitsvorbehalt}

Entsprechend Art. 6 Abs. 1 sowie Art. 9 Abs. 2 DSGVO gilt der Crundsatz des Verbots mit Erlaubnisvorbehalt. Eine Verarbeitung personenbezogener Daten ist daher nur zulässig, sofern sie sich auf einen entsprechenden Erlaubnistatbestand stützen kann.

Dieser Regelungsansatz spiegelt sich im nationalen Datenschutzrecht ebenfalls wider. Das Sozialrecht bringt die bereichsspezifische Ausprägung des Verbots mit Zulässigkeitsvorbehalt in $₫ 67 \mathrm{~b}$ Abs. 1 S. 1, 2 SGB X und $\$ 284$ Abs. 3 SGB V mit engeren Voraussetzungen zum Ausdruck. $\mathbb{5} 67 \mathrm{~b}$ Abs. 1 S. 1 SGB X setzt für die Zulässigkeit einer Verarbeitung das Eingreifen einer Erlaubnisnorm nach dem SCB voraus und wiederholt somit gewissermaßen das allgemeine Verbot mit Zulässigkeitsvorbehalt im Hinblick auf die Speicherung, Veränderung, Nutzung, Übermittlung, Einschränkung der Verarbeitung sowie Löschung von Sozialdaten und begrenzt gleichzeitig die in Betracht kommenden Rechtfertigungstatbestände. ${ }^{49}$ Diese können sich demnach nur aus dem Sozialgesetzbuch ergeben. Satz 2 bezieht dies auch auf besondere Kategorien von personenbezogenen Daten nach Art. 9 Abs. 1 DSGVO.

Auch die bereichsspezifische Norm des $\$ 284$ SGB V, die die zentrale Zweckbindungsnorm für Datenumgänge bei den Krankenkassen darstellt, macht deutlich, dass eine Verarbeitung von Sozialdaten nur zulässig ist, sofern dies - abgesehen von der ausdrücklichen Einwilligung des Betroffenen - durch eine Rechtsvorschrift des Sozialgesetzbuchs legitimiert ist. So dürfen Sozialdaten

46 Vgl. BT-Drs. 18/12611, S. 96.

47 BSG, Urteil vom 10.12.2008 - B 6 KA 37/07 R, juris-Rn. 35.

48 An dieser Stelle sei darauf hingewiesen, dass mit dem Entwurf zum 2. DSAnpUG-EU bei der Verarbeitung von biometrischen, genetischen oder Gesundheitsdaten formelle Wirksamkeitsvoraussetzungen für Einwilligungserklärungen geschaffen werden sollen, vgl. BT-Drs. 19/4674, S. 153. Hierzu bereits oben in Kap. 1.1.3.2.1.

49 Vgl. Bieresborn, in: von Wulffen/Schütze, SGB X, 8. Aufl., 2014, \$ 67b Rn. 3; Kircher, Der Schutz personenbezogener Gesundheitsdaten im Gesundheitswesen, 2016, S. 209. 
gemäß $₫ 284$ Abs. 3 S. 1 Hs. 1 SGB V grundsätzlich nur zu den Zwecken verarbeitet werden, zu denen sie gemäß $\$ 284$ Abs. 1 SGB V erhoben wurden. Darüber hinaus ist eine Verarbeitung der Sozialdaten zu anderen Zwecken nur zulässig, sofern eine Rechtsvorschrift des Sozialgesetzbuchs dies gestattet. Damit sind neben den Regelungen des SGB V (z.B. $\mathbb{2} 287$ SGB V) auch solche des allgemeinen Sozialdatenschutzrechts umfasst (z.B. $\$ 76 \mathrm{c}$ Abs. 2 Nr. 2 SCB X)..$^{50}$ Angesichts der Regelung in Art. 5 Abs. 1 lit. b) 2. HS DSGVO hat der Bundesgesetzgeber hier die Möglichkeit, das Zweckbindungsgebot zu lockern.

\subsection{Verhältnis von $S G B V$ zum allgemeinen Sozialdatenschutzrecht (SGB I und SGB X)}

Vor dem Hintergrund der Existenz eines allgemeinen (SCB I und SGB X) und eines speziellen (SGB V) bereichsspezifischen Datenschutzrechts stellt sich die Frage, ob die spezielleren Normen aus dem bereichsspezifischen Sozialdatenschutzrecht der Fachbücher des SGB (hier SGB V) eine Sperrwirkung hinsichtlich des Rückgriffs auf die Regelungen im allgemeinen Sozialdatenschutzrecht entfalten. Eine allgemeine Sperrwirkung der zulasten der Vorschriften des Sozialdatenschutzrechts im SGB X kommt nicht in Betracht, da die Normen des SGB V nur vereinzelte Datenumgänge regeln. Eine solche Sperrwirkung aus den nur punktuellen Regelungen des SGB V gegenüber dem allgemeinen Sozialdatenschutzrecht ergibt sich im Übrigen auch nicht aus der Rechtsprechung des Bundessozialgerichts vom 10.12.2008 ${ }^{51}$. Das Gericht schloss lediglich einen Rückgriff auf das BDSG a.F. oder Landesdatenschutzrecht aus, ${ }^{52}$ während es die Anwendung der allgemeinen Vorschriften des bereichsspezifischen Datenschutzrechts nach dem SGB X a.F. durchaus erwogen hat und nur deshalb im konkreten Fall nicht zur Anwendung gebracht hat, da es sich bei den betroffenen Leistungserbringern nicht um Stellen nach $\mathbb{} 35$ SGB I a.F. handelt. 53 Eine Sperrwirkung infolge eines Vorrangs nach dem Grundsatz „lex specialis derogat legi generali“ "kann sich ausschließlich für den jeweils mit der Norm des SGB V konkret geregelten Bereich ergeben. Im Übrigen sind die Normen des SGB V und des allgemeinen Sozialdatenschutzrechts gleichrangig. ${ }^{54}$

Tatbestände des allgemeinen Sozialdatenschutzrechts nach $\mathbb{} 35$ SGB I und $\mathbb{N} 57 \mathrm{ff}$. SGB X werden durch die fachspezifischen Datenschutzregelungen des SGB V ergänzt und hinsichtlich des spezifischen Regelungsgegenstandes der jeweiligen SGB V-Norm verdrängt.

\footnotetext{
50 Vgl. Didong/Koch, in: Schlegel/Voelzke, juris PK-SGB V, 3. Aufl. 2016, § 284 Rn. 18.

51 BSG, Urteil vom 10.12.2008 - B 6 KA 37/07 R.

52 BSG, Urteil vom 10.12.2008 - B 6 KA 37/07 R, juris-Rn. 33ff.

53 BSG, Urteil vom 10.12.2008 - B 6 KA 37/07 R, juris-Rn. 23, 33, 35.

54 BSG, Urteil vom 02.11.2010 - B 1 KR 12/10 R, juris-Rn. 18.
} 


\subsection{Art der Daten}

Die vom Leistungsträger erhobenen Abrechnungs- und Behandlungsdaten enthalten Informationen, die sich auf einen Versicherten und auf den jeweiligen Leistungserbringer und damit auf identifizierte oder identifizierbare natürliche Personen beziehen. Aus den Daten mit Versichertenbezug lassen sich Informationen über den Gesundheitszustand des Versicherten ableiten. Es handelt sich damit um Gesundheitsdaten im Sinne des Art. 4 Nr. 15 DSGVO, die als besondere Kategorien personenbezogener Daten im Sinne des Art. 9 Abs. 1 DSGVO wegen ihrer unterstellten besonderen Sensibilität nur unter erhöhten Voraussetzungen verarbeitet werden können.

\subsection{Auswertung als Datenverarbeitung}

Die Auswertung der Sozialdaten durch den Leistungsträger stellt einen Datenverarbeitungsvorgang im Sinne des $\$ 67 \mathrm{~b}$ SGB X dar. Hierbei ist zu berücksichtigen, dass anstelle der bisher in $\$ 67 \mathrm{~b}$ SGB X a.F. verwendeten Begriffe „Verarbeitung“ und „Nutzung“(vgl. $\$ 67$ Abs . 6, 7 SGB X a.F.) in der neuen Fassung von der „Speicherung, Veränderung, Nutzung, Übermittlung, Einschränkung der Verarbeitung und Löschung“ die Rede ist, weil „Verarbeitung“ nunmehr gemäß Art. 4 Nr. 2 DSGVO als Oberbegriff für alle Datenumgänge umfassender definiert ist als:

„jeden mit oder ohne Hilfe automatisierter Verfahren ausgeführten Vorgang oder jede solche Vorgangsreihe im Zusammenhang mit personenbezogenen Daten wie das Erheben, das Erfassen, die Organisation, das Ordnen, die Speicherung, die Anpassung oder Veränderung, das Auslesen, das Abfragen, die Verwendung, die Offenlegung durch Übermittlung, Verbreitung oder eine andere Form der Bereitstellung, den Abgleich oder die Verknüpfung, die Einschränkung, das Löschen oder die Vernichtung“

Die Auswertung der Sozialdaten durch den Leistungsträger beinhaltet insbesondere das Auslesen und Verwenden der Daten, um bestimmte Auswertungsergebnisse und Statistiken zu erzielen. Insoweit fällt die Auswertung der Sozialdaten jedenfalls unter den Verarbeitungsbegriff der DSGVO.

\ 67b SGB X soll nach der Gesetzesbegründung alle Verarbeitungsvorgänge „außerhalb der Erhebung“ erfassen. ${ }^{55}$ Bei der Anpassung des Sozialdatenschutzrechts an die DSGVO sollte der bisherige Anwendungsbereich der Ermächtigungsnorm beibehalten werden. ${ }^{56} \mathrm{Nach}$ bisherigem Recht war in $\$ 67$ Abs. 7 SGB X a.F. der Begriff „Nutzung“ neben den Begriffen „Erheben“ und „Verarbeiten“ als Auffangtatbestand enthalten. Wurden Sozialdaten mit einer

55 Vgl. BT-Drs. 18/12611, S. $111 f$.

56 Vgl. BT-Drs. 18/12611, S. 111. 
Zweckbestimmung ausgewertet, zusammengestellt oder auf sonstige Art zur Kenntnis genommen, lag ein Nutzen von Sozialdaten vor. ${ }^{57}$ Die neue Vorschrift ist daher so zu verstehen, dass die Auswertung der Sozialdaten durch den Leistungsträger jedenfalls unter den Begriff der Nutzung im Sinne des $\mathbb{5}$ 67 b SGB X fällt. Dass Art. 4 Nr. 2 DSGVO den Begriff der Nutzung nicht ausdrücklich erwähnt, dürfte unschädlich sein, da die dort genannten Unterfälle lediglich beispielhaft („wie“) sein dürften und die abweichende Begriffsnutzung im SGB jedenfalls dem Verarbeitungsbegriff unterfällt und daher den Anwendungsbereich der DSGVO nicht einschränkt.

\subsubsection{Gesetzliche Ermächtigung}

\subsection{Nutzung zu Forschungszwecken (S 287 SGB V)}

\$ 287 SGB V erlaubt den Krankenkassen - unter Einhaltung der nachfolgend weiter beschriebenen Voraussetzungen - die leistungserbringer- oder fallbeziehbare, selbst durchgeführte Auswertung ihrer Datenbestände für zeitlich befristete und im Umfang begrenzte Forschungsvorhaben. $\$ 287$ SGB V lautet:

„(1) Die Krankenkassen und die Kassenärztlichen Vereinigungen dürfen mit Erlaubnis der Aufsichtsbehörde die Datenbestände leistungserbringer- oder fallbeziehbar für zeitlich befristete und im Umfang begrenzte Forschungsvorhaben, insbesondere zur Gewinnung epidemiologischer Erkenntnisse, von Erkenntnissen über Zusammenhänge zwischen Erkrankungen und Arbeitsbedingungen oder von Erkenntnissen über örtliche Krankheitsschwerpunkte, selbst auswerten oder über die sich aus $\$ 304$ ergebenden Fristen hinaus aufbewahren.

(2) Sozialdaten sind zu anonymisieren."

\section{Forschungsvorhaben}

Unter Forschung versteht man die geistige Tätigkeit, mit dem Ziel, in methodischer, systematischer und nachprüfbarer Weise neue Erkenntnisse zu gewinnen. ${ }^{58}$ Sie erfasst insbesondere die Fragestellung der Methodik sowie die Bewertung des Forschungsergebnisses und seine Verarbeitung und bewirkt den Fortschritt der Wissenschaft. Wissenschaft ist wiederum jede Tätigkeit, die nach ihrem Inhalt und Form als ernsthafter planmäßiger Versuch zur Ermittlung der Wahrheit anzusehen ist. 59 \$ 287 SGB V nennt beispielhaft und somit nicht abschließend als zulässige Zwecke von Forschungsvorhaben die Gewinnung von

57 Vgl. Bieresborn, in: von Wulffen/Schütze, SGB X, 8. Aufl., 2014, \$67 Rn. 29.

58 Vgl. BT-Drs. V/4335, S. 4; Sifferdecker, in: Kasseler Kommentar, Sozialversicherungsrecht, 2017, § 287 SGB V Rn. 3 m.w.N.

59 BVerfG, Urteil vom 29.05.1973, Az.: 1 BvR 424/71. 
- epidemiologischen Erkenntnissen,

- Erkenntnissen über Zusammenhänge zwischen Erkrankungen und Arbeitsbedingungen,

- von Erkenntnissen über örtliche Krankheitsschwerpunkte.

\section{Nichtmedizinische Fragestellungen}

Die Frage, ob auch nichtmedizinische Fragestellungen Gegenstand der Forschungsvorhaben sein können, wird unterschiedlich beantwortet. Nach Kranig ist dies grundsätzlich zu bejahen, falls das Forschungsvorhaben geeignet ist, Erkenntnisse zu gewinnen, die die Krankenkassen bei Erfüllung ihrer gesetzlichen Aufgaben unterstützen. So seien Forschungen zur Wirksamkeit und Effizienz gesetzgeberischer Neuerungen, z.B. bei Vereinbarungen zur integrierten Versorgung ( $\$ \mathbb{S}$ 140a ff. SGB V) oder bei Einführung der elektronischen Gesundheitskarte ( $\$ 291$ SGB V) als zulässig anzusehen. Als Forschungszweck kämen demnach etwa die Verbesserung der getroffenen Maßnahmen oder das Erkennen von Nachbesserungsbedarf durch den Gesetzgeber in Betracht. ${ }^{60}$

Hornung verneint dagegen die Erstreckung auf nichtmedizinische Vorhaben mit der Begründung, dass diese nicht den verfassungsrechtlichen Rechtfertigungsgrund der Vermeidung von Krankheiten erfüllen, spezielle gesetzliche Regeln nicht umgangen werden dürfen und der Gesetzgeber mit den genannten Beispielen eine Typisierung vorgenommen habe. ${ }^{61}$ Dieser Rechtsauffassung kann u.E. jedoch aus folgenden Gründen nicht gefolgt werden:

Angesichts der ausdrücklich als nicht abschließend aufgezählten Regelbeispiele (,insbesondere“) müssen diese gerade nicht als typisierende Einschränkungen verstanden werden. Angesichts der Aufgaben der gesetzlichen Krankenversicherung sowie der Kassen(zahn)ärztlichen Vereinigungen, zu denen auch die Sicherstellung einer wirtschaftlichen Versorgung der Versicherten sowie eines effektiven Leistungsgeschehens gehören, legitimieren die Bestimmungen des $\mathbb{} 287$ SGB V eine Auswertung (und längere Aufbewahrung) auch für nichtmedizinische Forschung. ${ }^{62}$

Dies gilt auch angesichts der schwierigen Abgrenzbarkeit zwischen medizinischen und nichtmedizinischen Forschungszwecken in diesem Bereich, wie beispielsweise Politikfolgenforschung, die etwa auf Untersuchungen über

- Veränderungen in der Inanspruchnahme von Leistungen (z.B. nach Einführung der Praxisgebühr),

- Veränderungen in der Verordnungsweise (z.B. Wechsel zu verschreibungspflichtigen Präparaten aufgrund veränderter Erstattungsregelungen) oder

60 Kranig, in: Hauck/Noftz, SGB V, Stand: 08/2017, § 287 Rn. 6.

61 Hornung, in: Hänlein/Schuler, LPK-SGB V, 5. Aufl., 2016, § 287 Rn. 3.

62 Schifferdecker, in: Kasseler Kommentar, Sozialversicherungsrecht, 2017, \ 287 SGB V Rn. 4. 
- Verlagerungseffekte zwischen dem stationären und ambulanten Sektor (z.B. nach Einführung der DRGs)

gerichtet sind. ${ }^{63}$ Es werden hierdurch einerseits Erkenntnisse über die Wirksamkeit und Effizienz von Maßnahmen auf der regulatorischen, nicht-medizinischen Ebene gesammelt. Andererseits lassen sich auf Grundlage dieser Erkenntnis medizinisch begründete Maßnahmen zur Optimierung der medizinischen Versorgung einleiten. Dies gilt etwa auch für die sog. Wissenstransferforschung, die sich etwa mit der Frage befasst, wie schnell und durch welche Arztgruppen neue Verfahren und Behandlungsempfehlungen aufgegriffen werden bzw. welche Patientengruppen diese Behandlung erhalten. ${ }^{64}$

Insbesondere auch angesichts der Vorgaben der DSGVO zur weiten Auslegung der Forschungszwecke dürften auch nichtmedizinische Forschungszwecke vorliegend erfasst sein, soweit Erkenntnisse zur Förderung der öffentlichen Gesundheit gewonnen werden sollen und sonstige Voraussetzungen für die Datenverarbeitung erfüllt werden.

\section{Interne Fragestellungen}

Fraglich ist, ob eine Grenze im Rahmen des $\$ 287$ SGB V hinsichtlich zulässiger Forschungsvorhaben dort zu ziehen ist, wo die Krankenkasse die Erkenntnisse, die im Rahmen der Vorhaben gewonnen werden, nicht selbst bei Erfüllung ihrer gesetzlichen Aufgaben nutzen kann. Der Wortlaut der Norm erfasst zunächst nur, dass die tatsächliche Durchführung einer Auswertung durch die Krankenkasse selbst passieren muss, sodass jedenfalls eine Auswertung durch Dritte unzulässig ist. ${ }^{65} \mathrm{Ob}$ die Forschungsfrage ausschließlich krankenkassenintern sein muss, ergibt sich hieraus nicht. Nach Ansichten in der Literatur regelt $\mathbb{} 287$ SGB V aber die Selbstauswertung der Daten durch die gesetzlichen Krankenkassen zu internen, eigenen Forschungsvorhaben. ${ }^{66}$

Unter anderem wird vertreten, dass Externe als wissenschaftliche Begleitung des (internen) Forschungsprojekts des Leistungsträgers - etwa im Rahmen der Auftragsverarbeitung - hinzugezogen werden. ${ }^{67}$ Dies erscheint jedenfalls für Auftragsverarbeiter konsequent, da diese dem Verantwortlichen zugerechnet

63 Vgl. hierzu Schubert/Köster/Küpper-Nybelen/Ihle, Versorgungsforschung mit GKV-Routinedaten, Bundesgesundheitsblatt 2008, 1095 (1100).

$64 \mathrm{Vgl.Schubert/Köster/Küpper-Nybelen/Ihle,} \mathrm{Versorgungsforschung} \mathrm{mit} \mathrm{GKV-Routinedaten,} \mathrm{Bundesgesundheits-}$ blatt 2008, 1095 (1100).

65 Michels, in: Becker/Kingreen, SGB V, 5. Aufl., 2017, § 287 Rn. 3.

66 Vgl. Kranig, in: Hauck/Noftz, SGB V, Stand: 08/2017, § 287 Rn. 6; Schifferdecker, in: Kasseler Kommentar, Sozialversicherungsrecht, 2017, \287 SGB V Rn. 4; Michels, in: Becker/Kingreen, SGB V, 5 Aufl., 2017, \287 Rn. 1.

67 Auftrags(daten)verarbeitung als zulässig ansehend: Hornung, in: Hänlein/Schuler, LPK-SGB V, 5. Aufl., 2016, \287 Rn. 2.; a.A. Schifferdecker, in: Kasseler Kommentar, Sozialversicherungsrecht, 2017, § 287 SGB V Rn. 5. Forschungsinstitute, die von den Krankenkassen oder Kassenärztlichen Vereinigungen getragen werden, nicht als Dritte im Sinne der Norm ansehend: Wenner, in: Eichenhofer/Wenner, SGB V, 2013, § 287 Rn. 4; Schifferdecker, in: Kasseler Kommentar, Sozialversicherungsrecht, 2017, \$ 287 SGB V Rn. 5. 
werden können. Jedenfalls ist es unschädlich, dass vorliegend die konkrete Fragestellung für die Datenauswertung gemeinsam mit der externen Einrichtung entwickelt oder von dieser vorgegeben werden soll. Hierzu führt etwa Didong wie folgt aus:

„Die in \$287 SGB V vorgesehene eigene Auswertung der Datenbestände durch die Krankenkasse schließt eine wissenschaftliche Begleitung und Auswertung durch beigezogene Dritte nicht aus. Da der Gesetzgeber in $\$ 65$ SGB V die wissenschaftliche Auswertung von Modellvorhaben nach den ss 63, 64 SGB V durch unabhängige Sachverständige vorgesehen hat, ist kein Grund ersichtlich, warum im Rahmen von Forschungsvorhaben nach \$287SGB V die Auswertung allein durch Mitarbeiter der Krankenkassen oder Kassenärztlichen Vereinigung und nicht in Zusammenarbeit mit fachkundigen Dritten erfolgen soll. Hierdurch kann auch das Ziel von Forschungsvorhaben besser erreicht werden." ${ }^{6} 8$

Damit spricht erst recht nichts gegen die Einschaltung von externen Dritten zur Definition des Forschungsvorhabens. Die Auswertung soll vorliegend allein durch die Mitarbeiter des Leistungsträgers ohne Einschaltung Dritter erfolgen.

\section{Datenbestände}

Der Leistungsträger darf gemäß $\mathbb{2} 287$ SGB V ausschließlich auf bereits vorhandene Datenbestände zurückgreifen. Der Zugriff ist damit auf die bereits zu anderen Zwecken (nach $\mathbb{2} 284$ SGB V) in zulässiger Weise erhobenen und gespeicherten Daten beschränkt. ${ }^{69}$ Der Leistungsträger ist nicht befugt, darüberhinausgehende Daten zum Zweck der Forschung zu erheben. $\mathbb{2} 287$ SGB V beschränkt die auswertbaren Daten ferner auf leistungserbringerbeziehbare und fallbeziehbare Daten. Leistungserbringerbeziehbar sind Daten, die den Leistungserbringer nicht unmittelbar erkennen lassen, aber bestimmbar machen. Fallbeziehbar sind Daten eines Versicherten, außer den Angaben über persönliche oder sachliche Verhältnisse, welche die Bestimmung des Versicherten erlauben. Eine versichertenbeziehbare Auswertung für Forschungszwecke ist damit ausgeschlossen. Das bedeutet, dass nur Daten ausgewertet werden dürfen, die keine Rückschlüsse auf bestimmte Versicherte erlauben. ${ }^{70}$ Es reicht nicht aus, dass die Ergebnisse der Auswertung keine Rückschlüsse darauf zulassen. Selbstredend ist aus der Kombination von Angaben über einen Leistungserbringer und einen konkreten Fall - auch wenn identifizierende Daten wie Name, Adresse und Versichertennummer eines Versicherten nicht in die Auswertung einbezogen werden - eine Bestimmung dieser Person nicht

68 Didong, in: jurisPK-SGB V, 3. Aufl., 2016, $\$ 287$ Rn. 8.

69 Vgl. Didong, in: jurisPK-SGB V, 3. Aufl., 2016, \$287 Rn. 9.

70 Didong, in: jurisPK-SGB V, 3. Aufl., 2016, Rn. 9 m.w.N. 
völlig ausgeschlossen. Jedenfalls aber ist es auf Basis dieser Angaben nicht zulässig, Versichertenprofile auszuwerten, sondern nur einzelne Behandlungsfälle.

\section{Anonymisierung, \$287 Abs. 2 SGB V}

Die Sozialdaten müssen daher anonymisiert werden, wie es $\mathbb{2} 287 \mathrm{Abs} .2$ SGB V fordert. ${ }^{11}$ Hierdurch soll insbesondere erreicht werden, dass sie nicht mehr versichertenbeziehbar sind und damit keine Bestimmung eines Versicherten erlauben..$^{72}$ Der Zeitpunkt der Anonymisierung ist im Gesetz nicht bestimmt. Entsprechend dem Regelungszweck, bei Forschungsvorhaben nur mit anonymisierten Daten zu arbeiten, ist aber davon auszugehen, dass die Anonymisierung der Sozialdaten spätestens dann erfolgen muss, sobald sie zum Zwecke der Auswertung in das Forschungsvorhaben einbezogen werden. ${ }^{73}$

Die Anonymisierung der Daten muss also vor der Auswertung erfolgen, die Daten dürfen nicht personenbezogen ausgewertet werden. ${ }^{74}$

\section{Auslegung des Begriffs der Anonymisierung}

Es ist angesichts der Anforderungen an eine Anonymisierung $75 \mathrm{zu}$ berücksichtigen, dass vorliegend die Auswertung der Daten durch die Stelle erfolgt, die diese Daten - mit Personenbezug - selbst erhoben hat.

Eine auch nur faktische Anonymisierung der Daten durch Aufhebung des Personenbezugs ist - selbst bei relativem Verständnis des Personenbezugs - nicht möglich, da der Leistungsträger in seiner Gesamtheit grundsätzlich stets $\mathrm{Zu}$ griff auf alle seine Datenbestände hat und also die Möglichkeit einer De-Anonymisierung stets gegeben sein wird. D.h., wenn der Leistungsträger vollständige, personenbezogene Datensätze zu einem Zweck vorliegen hat und diese Daten nun zusätzlich in einen zweiten, davon getrennten Datensatz zu Forschungszwecken ohne identifizierende Daten speichert, den ggf. ein anderer Mitarbeiter oder eine andere Abteilung bearbeitet, kann dies nach dem bisherigen Verständnis des Begriffs der Anonymisierung (vgl. $\mathbb{S} 67 \mathrm{Abs} .8$ SGB X a.F.) nicht ausreichen, da das Wissen der gesamten verantwortlichen Stelle maßgeblich ist und nicht nur das einzelner Mitarbeiter. Eine Anonymisierung ist deswegen nur hinsichtlich solcher Daten möglich, die der Leistungsträger für die erhobenen Zwecke nicht mehr benötigt und deswegen

\footnotetext{
71 Dieses Erfordernis könnte bereits hinsichtlich der Daten, die den Krankenkassen nach $\S 295$ Abs. 1 b S. 1 SGB V übermittelt werden, aus $\S 284$ Abs. 3 S. 2 Hs. 2 SGB V folgen, wonach ein „Versichertenbezug vorher“ - also vor der zweckändernden Weiterverwendung - „zu löschen“ ist. Das betrifft Daten, die nicht über die Kassenärztlichen Vereinigungen, sondern unmittelbar von Leistungserbringern an die Krankenkassen übermittelt werden.

72 Vgl. Kranig, in: Hauck/Noftz, SGB V, Stand: 08/2017, § 287 Rn. 8.

73 Leber, in: Orlowski/Rau/Schermer/Wasem/Zipperer, GKV-Kommentar, SGB V, Stand: 08/2017, \$287 Rn. 9.

74 Michels, in: Becker/Kingreen, SGB V, 5 Aufl., 2017, \$287 Rn. 5.

$75 \mathrm{Vgl}$. den entsprechenden Abschnitt im Gutachtenteil von Roßnagel im vorliegenden Buch.
} 
löschen kann bzw. muss. Nur wenn davon ausgegangen werden kann, dass das zurechenbare Wissen nicht mehr bei der jeweiligen Krankenkasse vorhanden ist, kann nach bisherigem Verständnis des Begriffs der Anonymisierung eine Auswertung auf Grundlage des $\mathbb{2} 287$ SGB V erfolgen. Wann eine solche Löschung regelmäßig durchzuführen ist, bestimmt $\$ 304$ SGB V. Danach sind die Sozialdaten grundsätzlich zu löschen, soweit ihre Kenntnis für die Aufgabenerfüllung des Leistungsträgers nicht mehr erforderlich ist. Lediglich eine Anonymisierung von Daten aus bereits abgeschlossenen Vorgängen und damit von „Altbeständen“ ist daher möglich. Dies eröffnet jedoch ausschließlich die Möglichkeit der Nutzung von Altbeständen für Forschungsvorhaben, die sich auf die in Vergangenheit abgeschlossene Fälle beziehen. ${ }^{76}$ Eine Anonymisierung der aktuellen Daten des Leistungsträgers, die laufend neu erhoben werden, ist angesichts der Forderung nach jedenfalls faktischer Anonymisierung nicht möglich.

Aus dieser Problematik ergeben sich zwei gegenläufige Rechtsauffassungen über die Reichweite der Erlaubnisnorm in $\mathbb{2} 287$ SGB V:

1. Die Erlaubnisnorm soll nur die Auswertung von Altdatenbeständen ermöglichen.

2. Die Erlaubnisnorm ermöglicht auch die Auswertung nicht abgeschlossener Sachverhalte.

Die erstgenannte Ansicht orientiert sich strikt an dem Wortlaut („Anonymisierung“) und wird etwa von Schifferdecker vertreten, der hierzu ausführt, dass die Forschungsvorhaben sich nur auf bereits abgeschlossene Vorgänge beziehen können. ${ }^{77}$ Andere schließen sich der zweitgenannten Ansicht an und sehen die Auswertung aller - auch aktueller - Datenbestände als zulässig an, wobei - auch unter den Befürwortern dieser Ansicht - grundsätzlich Einigkeit insofern besteht, als auch diese Daten dem Forschungsprojekt nur in anonymisierter Form zugeführt werden dürfen. ${ }^{78}$

\section{Modifiziertes Verständnis der Anonymisierung}

Im Sinne der zweitgenannten Ansicht wird zur Ermöglichung der Auswertung aktueller Fälle, in denen laufend neue Daten erhoben werden, ein modifiziertes Verständnis von der Anonymisierung im Sinne des $\$ 287$ Abs. 2 SGB V entwickelt.

Vorgeschlagen wird insbesondere ein Verschlüsselungsverfahren, bei dem die Verschlüsselungskennziffern den Personen, die im Forschungsvorhaben selbst tätig sind, nicht zur Verfügung gestellt werden dürfen. Die Daten sind dann

76 Vgl. Kranig, in: Hauck/Noftz, SGB V, Stand: 08/2017, § 287 Rn. 10.

77 Schifferdecker, in: Kasseler Kommentar, Sozialversicherungsrecht, 2017, § 287 SGB V Rn. 8.

78 Vgl. Didong, in: jurisPK-SGB V, 3. Aufl., 2016, Rn. 12. 
aus Sicht dieser natürlichen Person - im Sinne eines relativen Personenbezugs anonymisiert. Die am Forschungsprojekt tätigen Personen dürfen hierfür keine Zugriffsrechte auf die Kennziffer und kein rechtliches Mittel zur De-Anonymisierung haben. Hierzu führt Kranig wie folgt aus:

„Eserscheint hingegen möglich, durch Verwendung von leistungserbringer- oder fallbeziehbaren Kennziffern oder mittels anderer Verschlüsselungsverfahren den Datenfluss vom laufenden Behandlungsfall zum Forschungsvorhaben sicherzustellen, ohne dass Personen, die im Forschungsvorhaben tätig sind, den konkreten Bezug zu einem bestimmten Leistungserbringer oder sogar zu einem bestimmten Versicherten herstellen können.“79

Mit ähnlicher Intention führt Michels in diesem Zusammenhang aus:

„Bei der Zusammenführung unterschiedlicher Datenbestände und in Fällen der zeitlich befristeten Verlaufskontrolle wäre damit eine Zusammenführung leistungserbringer- o. fallbezogener Daten nur im Wege einer Pseudonymisierung (...) außerhalb des Forschungsvorhabens mögl.“"80

Gegen ein solches, modifiziertes Verständnis der Anonymisierung im Sinne des $\mathbb{2} 287$ Abs. 2 SGB V, wonach eine Pseudonymisierung genügte, argumentiert etwa Hornung wie folgt:

„Für Forschungsvorhaben an derartigen noch nicht abgeschlossenen Fällen wird teilweise eine Pseudonymisierung vorgeschlagen, z.B. durch Verschlüsselung der Versicherungsnummer (Maaßen/Piepersberg, BArbBl Nr. 4 1989, 48; Kranig in Hauck/ Noftz, \$287 Rn 11), ggf. mit Absicherung der Reindividualisierung gemäß $\$ 286$ Abs. 3 (s.a. Roß, 3. Auflage, Rn 4). Dies ist strikt abzulehnen (wie hier Krauskopf-Waschull, SozKV, $\$ 287$ Rn 20; Fischinger in Spickhoff, Medizinrecht, $\$ 287 \mathrm{Rn} 3$; Schäfer in Berchtold/Huster/Rehborn, $\$ 287 \mathrm{Rn}$ 6). Schon definitorisch ist zwischen Anonymisieren (\$ 67 Abs. 8 SCB X) und Pseudonymisieren (\$ 67 Abs. 8a SGB X) zu unterscheiden. Wenn sich der Gesetzgeber dazu entschieden hat, explizit eine der beiden Varianten zuzulassen, so kann dies nicht mit Argumenten praktischer Notwendigkeit überspielt werden (s. Hornung/Roßnagel, 395ff.)." ${ }^{\text {"1 }}$

Auch Spindler verlangt eine strikte Anonymisierung und lässt eine Pseudonymisierung im Rahmen des $\$ 287$ Abs. 2 SGB nicht genügen. ${ }^{82}$

79 Kranig, in: Hauck/Noftz, SGB V, Stand: 08/2017, \287 Rn. 11.

80 Michels, in: Becker/Kingreen, SGB V, 5 Aufl., 2017, \$287 Rn. 5.

81 Hornung, in: Hänlein/Schuler, LPK-SGB V, 5. Aufl., 2016, \$287 Rn. 7.

82 Vgl. Spindler, MedR 2016, 691 (698). 


\title{
Stellungnahme
}

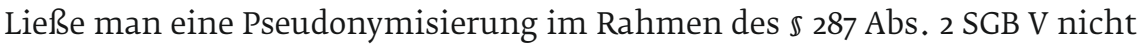
genügen, würde die Auswertung der Daten zu Forschungszwecken hinsichtlich nicht abgeschlossener Fälle und damit insbesondere hinsichtlich verlaufsbezogener Untersuchungen und Langzeitstudien in der Regel ausscheiden. Dies wiederum widerspräche den vom Gesetzgeber genannten Beispielen, die gem. $\mathbb{2} 287$ Abs. 1 SGB V erfasst sein sollen. Hierzu führt Kranig aus:

\begin{abstract}
„Derartige verlaufsbezogene Forschungsvorhaben sind gerade für die beispielhaft im Gesetz genannten Zwecke der epidemiologischen Untersuchungen der Untersuchung arbeitsbedingter Erkrankungen besonders wichtig. Es kann nicht davon ausgegangen werden, dass der Gesetzgeber diese verlaufsbezogenen Forschungsvorhaben unterbinden wollte. Weder ist dies daraus zu schließen, dass nur die Auswertung (vorhandener) Datenbestände zulässig ist (vgl. Rz 7), denn dies soll nur die Datenerhebung eigens für Forschungsvorhaben verhindern; noch ergibt sich dies daraus, dass die Forschungsvorhaben zeitlich befristet sein müssen; vielmehr deutet gerade die zeitliche Befristung von Forschungsvorhaben darauf hin, dass diese sich nicht auf in der Vergangenheit abgeschlossene Fälle beschränken müssen. Sind mithin verlaufsbezogene Forschungsvorhaben grundsätzlich zulässig, so müssen sie auch - unter Wahrung der datenschutzrechtlichen Gesichtspunkte - sinnvoll durchführbar sein. Wenn Kramer, GK-SGB V, $\mathbb{2} 287 \mathrm{Rz} 4$, im Anschluss an die Ausführungen zur Anonymisierung der Daten ausführt, ,eine sinnvolle leistungserbringerbeziehbare Auswertung der Datenbestände (komme) damit praktisch kaum in Betracht', so wird dies dem Willen des Gesetzgebers, interne Forschungsvorhaben der Krankenkassen und Kassen(zahnärztlichen) Vereinigung unter Wahrung des Datenschutzes zu ermöglichen, nicht gerecht." 83
\end{abstract}

Die Argumentation ist insofern überzeugend, als gerade die epidemiologischen Untersuchungen und solche über arbeitsbedingte Erkrankungen verlaufsbezogene Forschung unter Zugriff auf die laufende leistungserbringeroder fallbezogene Datenerhebung erfordern. Es geht hierbei um Untersuchungen hinsichtlich Verbreitung sowie Ursachen und Folgen von gesundheitsbezogenen Zuständen und Ereignissen in Bevölkerungen und Populationen. So schreiben Schubert/Köster/Küpper-Nybelen/Ihle zu Nutzungsmöglichkeiten der GKV-Routinedaten im Rahmen der Forschung u.a.:

„Mithilfe von versichertenbezogenen pseudonymisierten Bestandsdaten lassen sich aufgrund des vorhandenen Bevölkerungsbezugs administrative Prävalenz- und Inzidenzschätzungen nach soziodemographischen Variablen - in der Linie nach Alter und Geschlecht - anhand der ambulanten und/oder stationären Diagnosen vornehmen und auf Vergleichspopulationen standardisieren. (...) Auf der Basis kontinuierlich erhobener Daten sind Fortschreibungen dieser administrativen Prävalenzen und

83 Kranig, in: Hauck/Noftz, SGB V, Stand: 08/2017, \$ 287 Rn. 11 m.w.N. 
Inzidenzen und - bei Betrachtung mehrerer Jahre-Berechnungen des Alterseffektes möglich." ${ }^{4}$

sowie:

„Des Weiteren sind Versorgungsmuster von Interesse, zu deren Darstellung es längsschnittlich erhobener Daten über einen längeren Zeitraum bedarf (z.B. Definition und Beobachtung einer Kohorte von Versicherten)." ${ }^{85}$

und:

„Versorgungsforschung befasst sich sowohl mit den Folgen gesetzlicher und ordnungspolitischer Maßnahmen für das Versorgungssystem (Stichwort: Politikfolgenforschung) als auch mit dem Transfer wissenschaftlicher Erkenntnisse in den Alltag der Versorgung. Hier kommt den über einen längeren Zeitraum vorliegenden Routinedaten ebenfalls ein hoher Stellenwert zu, da auch nach Einführung einer Maßnahme (z.B. DMP, DRG, Praxisgebühr, Warnhinweise für Arzneimittel, Implementierung von Leitlinien, Rabattverträge) Daten für einen Zeitraum oder Zeitpunkt vor der Maßnahme/ dem zu beobachtenden Ereignis (rückwirkend) erhoben und die Auswirkungen aufdie Inanspruchnahme oder Art der Leistungen beschreiben und je nach Studiendesign auch kausal erklärt werden können." ${ }^{6}$

Diese Ausführungen zeigen beispielhaft, dass die Bestandsdaten des Leistungsträgers gerade auch durch den Rückgriff auf den fortwährenden Bestand mit jeweils neuen Daten besondere Vorteile im Rahmen von verlaufsbezogenen Forschungen bieten, dadurch die Gesetzgeber intendierten Langzeitbeobachtungen überhaupt erst möglich sind sowie retro- und prospektive Beobachtungen durchgeführt werden können. Dabei liegt der Vorteil der Daten von gesetzlichen Krankenkassen insbesondere auch in ihrer Vollständigkeit und in der fehlenden Selektion in Bezug auf etwa eine Verweigerung der Datennutzung, sodass über alle Versicherten Aussagen getroffen werden können. ${ }^{87}$ Hierzu Leber:

„Eine - je nach den Besonderheiten des Forschungszwecks unter Umständen sogar unverzichtbare - reversible Anonymisierung ist daher zulässig. Die Verpflichtung zur Anonymisierung von Sozialdaten erschwert die Durchführung von Forschungsvorhaben, die behandlungsfallbezogene Verlaufsbeobachtungen über einen

84 Schubert/Köster/Küpper-Nybelen/Ihle, Versorgungsforschung mit GKV-Routinedaten, Bundesgesundheitsblatt 2008, 1095 (1096).

85 Schubert/Köster/Küpper-Nybelen/Ihle, Versorgungsforschung mit GKV-Routinedaten, Bundesgesundheitsblatt 2008, 1095 (1098).

86 Schubert/Köster/Küpper-Nybelen/Ihle, Versorgungsforschung mit GKV-Routinedaten, Bundesgesundheitsblatt 2008, 1095 (1100).

87 Schubert/Köster/Küpper-Nybelen/Ihle, Versorgungsforschung mit GKV-Routinedaten, Bundesgesundheitsblatt 2008, 1095 (1102). 
längeren Zeitraum hinweg zum Gegenstand haben, da die Zuordnung neu hinzutretender Behandlungsdaten zu bereits anonymisierten Daten des Behandlungsfalls ohne Kenntnis des Personenbezugs nicht möglich ist. Insoweit ist eine datentechnisch mögliche Verschlüsselung des Personenbezuges - etwa durch getrennt zu speichernde personenbezogene Merkmale - geboten, die eine Deanonymisierung ausschließlich zum Zwecke der behandlungsfallbezogenen Zuordnung unter datenschutzrechtlich abgesicherten Vorkehrungen gegen Missbrauchsmöglichkeiten zulässt. Die Einzelheiten eines solchen Verfahrens sind vor allem im Hinblick auf einen ausreichenden Schutz der Sozialdaten mit der Aufsichtsbehörde (...) - zweckmäßigerweise unter Beteiligung des zuständigen Datenschutzbeauftragten - abzustimmen.“88 [Hervorhebung nicht im Original]

Vor diesem Hintergrund erscheint es u.E. sachgerecht, die Frage nach den Anforderungen der Anonymisierung im Sinne des $\mathbb{2} 287$ Abs. 2 SGB V jeweils aus dem Blickwinkel der die Daten auswertenden Person bzw. Abteilung zu beurteilen. Wird den Personen bzw. der Abteilung, die das Forschungsvorhaben durch die Auswertung der Daten durchführen, kein Mittel zur Re-Identifizierung (De-Anonymisierung) der Daten zur Verfügung gestellt, so handelt es sich aus deren Sicht um anonyme Daten. Dieses Ergebnis entspricht nicht nur den oben erwähnten praktischen Bedürfnissen, sondern auch der real gelebten Wirklichkeit innerhalb eines Leistungsträgers mit unterschiedlichen Abteilungen, die jeweils unterschiedliche Aufgaben zu erfüllen haben.

Zudem ist eine entsprechende Handhabung, bei der zwischen unterschiedlichen Stellen und Personen innerhalb eines Amtes unterschieden wird, etwa aus den Vorgaben zur Geheimhaltung über Personendaten, die für eine Bundesstatistik gemacht werden, grundsätzlich bekannt (\$16 Abs. 2 S. 1 Bundesstatistikgesetz).

$\mathrm{Zu}$ fordern ist aber angesichts der bestehenden Unsicherheiten in jedem Fall eine räumlich, organisatorisch und personell eigenständige Forschungsabteilung des Leistungsträgers. In dieser Forschungsabteilung tätigen Personen dürfen etwa nicht auch in den anderen Abteilungen des Leistungsträgers eingesetzt werden und im Zusammenhang mit anderen Aufgaben Zugriff auf die nicht anonymisierten Daten haben.

\section{Erlaubnis der Aufsichtsbehörde}

Die Auswertung der Daten für Forschungsvorhaben bedarf der vorherigen Erlaubnis der Aufsichtsbehörde. Dies wird ausführlich in Kapitel 1.4.5 behandelt. 


\section{Zwischenergebnis und Vereinbarkeit mit der DSGVO}

Unter Beachtung der aufgezeigten Voraussetzungen ist die Datenverarbeitung durch den Leistungsträger für Forschungszwecke damit gemäß $\mathbb{S} 67 \mathrm{~b}$ Abs. 1

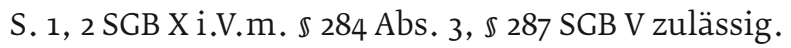

Art. 9 Abs. 2 DSGVO erlaubt gerade für den Gesundheitssektor die Verarbeitung sensibler Daten bei einem erheblichen öffentlichen Interesse, wozu insbesondere wissenschaftliche Forschung (lit. j) gehört. Die dargestellten strengen Anforderungen an die Auswertung der Daten durch den Leistungsträger sowie die von diesem nach Art. 89 Abs. 1 DSGVO zu gewährenden Garantien tragen der besonderen Sensibilität dieser Daten bei der Verarbeitung zu Forschungszwecken Rechnung.

Es gibt keine Anhaltspunkte für eine Unvereinbarkeit der Erlaubnisvorschrift in $\mathbb{2} 287$ SGB V mit der DSGVO und daher auch keinen Anlass für Änderungen des $\mathbb{2} 287$ SGB V zur Anpassung an die DSGVO. ${ }^{89}$ Insbesondere dürfen die Mitgliedsstaaten den Schutzstandard der DSGVO verändern und die Verarbeitung gesundheitsbezogener Daten beschränken (Art. 9 Abs. 4 DSGVO).

Die Bundesrepublik Deutschland wäre aber auch nicht daran gehindert, die Bestimmungen des $\mathbb{2} 287 \mathrm{SGB} V$ zugunsten der medizinischen Forschung - wiederum unter Berücksichtigung der Vorgaben der DSGVO (insbesondere des Art. 89 DSGVO) - zu lockern. ${ }^{90}$ Angesichts der in Folge der aufgezählten Beschränkungen restriktiven Möglichkeiten der Datenauswertung im Rahmen dieser Erlaubnisvorschrift hat bereits Leber 2005 unter Verweisung auf die geschafften verbesserten Bedingungen für die Beteiligten zur Datenverarbeitung und -nutzung nach den Datentransparenzregelungen in $\mathbb{S}$ 303a bis $303 \mathrm{f} S \mathrm{SGB} V$ die praktische Bedeutung von $\$ 287$ SGB V infrage gestellt. ${ }^{91}$

Jedenfalls wäre es wünschenswert, wenn der Gesetzgeber den Streit um die Bedeutung der Anonymisierung gemäß $\mathbb{2} 287$ Abs. 2 SGB beilegen würde und die Anforderungen hieran dahingehend klarstellte, dass die nicht abgeschlossenen Sachverhalte erfasst sind und beispielsweise nicht mehr der Begriff der Anonymisierung verwendet wird. Stattdessen könnte eine umschreibende Formulierung verwendet oder der Begriff der „formalen“ Anonymisierung, dem Vorbild des $₫ 5$ a Abs. 3 Bundesstatistikgesetzes folgend, eingeführt werden.

\subsection{Weitergehende Nutzung zu Forschungszwecken (\$ 67c Abs. 2 Nr. 2 SGB X)}

Es stellt sich die Frage, ob eine Nutzung der Sozialdaten zu Forschungszwecken durch die Krankenkassen auch über den Rahmen des $\mathbb{2} 287$ SGB V hinaus

89 Vgl. auch Schifferdecker, in: Kasseler Kommentar, Sozialversicherungsrecht, 2017, § 287 SGB V Rn. 2.

90 Auch Spindler, MedR 2016, 691 (699).

91 Leber, in: Orlowski/Rau/Schermer/Wasem/Zipperer, GKV-Kommentar, SGB V, Stand: 08/2017, § 287 Rn. 10. 
zulässig ist. In Betracht käme hier eine Verarbeitung personenbezogener Sozialdaten nach $\$ 67$ c Abs. 2 Nr. 2 SGB X.

Wie oben bereits ausgeführt, kommt den spezielleren Normen aus dem bereichsspezifischen Sozialdatenschutzrecht der Fachbücher des SCB (insb. SGB V) keine generelle Sperrwirkung bezüglich der Vorschriften des SGB I und SCB X zu. Vielmehr gehen die Regelungen des SGB V den allgemeinen Bestimmungen nur vor, soweit sie einen einzelnen Teilbereich spezifisch regeln. In diesem Zusammenhang ist fraglich, inwieweit $\$ 287$ SGB V eine Sperrwirkung für den Bereich der wissenschaftlichen Forschung mit Sozialdaten durch die Krankenkassen bewirkt und eine parallele Anwendbarkeit des $567 \mathrm{c}$ Abs. 2 Nr. 2 SCB X ausschließt. $\$ 287$ SCB X regelt die Voraussetzungen für die Verwendung der vorhandenen Datenbestände durch den Leistungsträger selbst. Nach verbreiteter Meinung der Literatur stellt $\$ 287$ SGB V eine lex specialis für den Bereich der Eigenforschung der Krankenkassen mit Sozialdaten, insbesondere im Verhältnis zu $\mathbb{S} 67 \mathrm{c}$ Abs. 5 SGB X dar. ${ }^{92}$ Ein Rückgriff auf die allgemeinen Verarbeitungstatbestände zur Legitimation der Verarbeitung weiterer personenbezogener Daten zu Forschungszwecken schiede damit aus.

Das Verhältnis der beiden Normen ließe sich auch anders interpretieren, denn der Grundsatz des „lex specialis derogat legi generali“ fordert eine Tatbestandskongruenz. Nur dann kann die allgemeinere Regel nicht mehr zur Anwendung gebracht werden. Da $\$ 287$ SGB V aber nicht nur erhöhte Anforderungen an die Verarbeitung zu Forschungszwecken aufstellt, sondern zugleich eine „leistungserbringer- oder fallbeziehbare“ Auswertung ermöglicht und zudem nur den Fall der Selbstauswertung regelt, könnte angenommen werden, dass der Fall einer nicht „leistungserbringer- oder fallbezogenen“ Fremdforschung durch die Norm gerade nicht geregelt und daher auch ein Rückgriff auf die allgemeinen Forschungstatbestände des SGB X nicht ausgeschlossen sein muss. Von der Literatur wird eine solche Auslegung bisher nicht gestützt, allerdings wird ihr auch nicht direkt widersprochen.

Betrachtet man dies vor dem Hintergrund der geänderten datenschutzrechtlichen Rahmenbedingungen durch die DSGVO, die eine weitgehende Privilegierung der wissenschaftlichen Forschung zum Leitprinzip erklärt, könnte auch dieser neue Rechtsrahmen Anlass zu einer verarbeitungsfreundlicheren Interpretation des $\$ 287$ SGB V bieten. Vom Willen des deutschen Gesetzgebers kann diese Interpretation freilich noch nicht gedeckt sein. Im Zuge einer zu erwartenden Anpassungsgesetzgebung könnte der Gesetzgeber aber den Weg für eine weitergehende Verarbeitung öffnen. Bis dahin wird man mit der wohl herrschenden Meinung einen Rückgriff auf $\$$ 67c SGB X bezüglich Forschungsvorhaben verneinen können. Infolge dieser Sperrwirkung käme $\$$ 67c Abs. 2

92 Vgl. Schifferdecker, in: Kasseler Kommentar, Sozialversicherungsrecht, 2017, \$287 SGB V Rn. 2; Leber, in: Orlowski/Rau/Schermer/Wasem/Zipperer, GKV-Kommentar, SGB V, Stand: 08/2017, \287 Rn. 1. 
Nr. 2 SGB X vorliegend lediglich für die Auswertung der Sozialdaten zur Planung im Sozialleistungsbereich in Betracht.

\subsection{Nutzung zu Planungszwecken (\$ 67c Abs. 2 Nr. 2 SGB X)}

Gemäß $\mathbb{5} 67$ c Abs. 2 Nr. 2 SGB X dürfen die nach Abs. 1 gespeicherten Daten von dem Leistungsträger für andere als die gespeicherten Zwecke genutzt werden, wenn es zur Durchführung eines bestimmten Vorhabens der Planung im Sozialleistungsbereich erforderlich ist und die Voraussetzungen des $\mathbb{5} 75$ Abs. 1, 2 oder 4a S. 1 SGB X X ${ }^{93}$ vorliegen. ${ }^{94}$

\section{Planung im Sozialleistungsbereich}

\$ 67c Abs. 2 Nr. 2 SGB X ermöglicht die Nutzung zu bestimmten Vorhaben der Planung im Sozialleistungsbereich. Sozialleistungen sind die im SGB vorgesehenen Dienst-, Sach- und Geldleistungen. Der Begriff der Planung umschreibt die Festlegung künftigen Verhaltens auf der Grundlage der Abschätzung künftiger Tatsachen anhand gegenwärtiger oder vergangener Tatsachen. Die Auswertung der Tatsachen muss stets zu einem bestimmten Zweck erfolgen. ${ }^{95}$

\section{Erforderlichkeit}

Die Nutzung der zu anderen Zwecken erhobenen und gespeicherten Daten kann für ein bestimmtes Planungsvorhaben im Sozialleistungsbereich erfolgen, wenn sie für das Planungsvorhaben erforderlich ist. Angesichts der

93 S 75 Abs. 1 SGB X lautet: „Eine Übermittlung von Sozialdaten ist zulässig, soweit sie erforderlich ist für ein bestimmtes Vorhaben

1. der wissenschaftlichen Forschung im Sozialleistungsbereich oder der wissenschaftlichen Arbeitsmarkt- und Berufsforschung oder

2. der Planung im Sozialleistungsbereich durch eine öffentliche Stelle im Rahmen ihrer Aufgaben und schutzwürdige Interessen der betroffenen Person nicht beeinträchtigt werden oder das öffentliche Interesse an der Forschung oder Planung das Geheimhaltungsinteresse der betroffenen Person erheblich überwiegt. Eine Übermittlung ohne Einwilligung der betroffenen Person ist nicht zulässig, soweit es zumutbar ist, ihre Einwilligung einzuholen. Angaben über den Namen und Vornamen, die Anschrift, die Telefonnummer sowie die für die Einleitung eines Vorhabens nach Satz 1 zwingend erforderlichen Strukturmerkmale der betroffenen Person können für Befragungen auch ohne Einwilligungen übermittelt werden. Der nach Absatz 4 Satz 1 zuständigen Behörde ist ein Datenschutzkonzept vorzulegen."

\ 75 Abs. 2 SGB X lautet: "Ergibt sich aus dem Vorhaben nach Absatz 1 Satz 1 eine Forschungsfrage, die in einem inhaltlichen Zusammenhang mit diesem steht, können hierzu auf Antrag die Frist nach Absatz 4 Satz 5 Nummer 4 zur Verarbeitung der erforderlichen Sozialdaten verlängert oder eine neue Frist festgelegt und weitere erforderliche Sozialdaten übermittelt werden."

\$ 75 Abs. 4a Satz 1 SGB X lautet: „Ergänzend zur Übermittlung von Sozialdaten zu einem bestimmten Forschungsvorhaben nach Absatz 1 Satz 1 kann die Verwendung dieser Sozialdaten auch für noch nicht bestimmte, aber inhaltlich zusammenhängende Forschungsvorhaben des gleichen Forschungsbereiches beantragt werden."

94 Dem Rückgriff auf $\$ 67$ c Abs. 2 Nr. 2 SGB X steht auch $\$ 284$ Abs. 3 S. 1 SGB V nicht entgegen, da dieser keine Sperrwirkung hinsichtlich allgemeinerem Datenschutzrecht des SGB X entfaltet.

95 Vgl. Rombach, in: Hauck/Noftz, SGB X, Stand: 08/2017, § 75 Rn. 18. 
weiten Fassung der Erlaubnisvorschrift kommt der Prüfung der Erforderlichkeit eine besondere Bedeutung $\mathrm{zu} .{ }^{96}$

Aus der Erforderlichkeit ergibt sich bereits die Eingrenzung des Datenumfangs. So ist die Nutzung individualisierbarer Daten nicht zulässig, soweit eine Anonymisierung bzw. Aggregierung der Daten zumutbar ist. Diese Voraussetzung wird im Rahmen des Szenarios bereits dadurch eingehalten, dass die Daten vor der Auswertung anonymisiert werden (im Sinne des modifizierten Verständnisses der Anonymisierung innerhalb des Leistungsträgers, hierzu s.o.).

Ferner verlangt die Erforderlichkeit die Begrenzung auf Sozialdaten, die der Planer in der betreffenden Phase des Planungsvorhabens unbedingt haben muss. 97

\section{Interessenabwägung (S 75 Abs. 1 S. 1 SGB X)}

Für die Nutzung zu Planungszwecken müssen die Voraussetzungen von $\mathbb{} 75$ Abs. 1 SGB X vorliegen. Insbesondere dürfen schutzwürdige Interessen des Betroffenen nicht beeinträchtigt sein oder das öffentliche Interesse an der Planung muss das Geheimhaltungsinteresse der betroffenen Person erheblich überwiegen. Abzuwägen sind daher nicht nur Beeinträchtigungen des einzelnen Betroffenen, sondern auch aller Betroffenen zusammen gegen den Nutzen, der aus der Durchführung des Planungsvorhabens zu erwarten ist. ${ }^{98}$

Bei Vorliegen entsprechender Anhaltspunkte ist in jedem Einzelfall eine mögliche Beeinträchtigung zu prüfen. ${ }^{9} \mathrm{Zu}$ berücksichtigen sind sowohl objektive als auch subjektive Gesichtspunkte. Hierbei ist eine Prognose über mögliche Folgen einer Datennutzung für den Betroffenen zu stellen. Schutzwürdige Belange können demnach beeinträchtigt werden, wenn aus objektiver Sicht unter Zugrundelegung durchschnittlicher Verhältnisse Nachteile für den Betroffenen zu befürchten sind (z.B. als sensitive Zusatzinformationen, dass der Aufenthalt des Betroffenen in einem Landekrankennaus feststellbar ist). ${ }^{100}$ Eine Beeinträchtigung kommt auch in Betracht, wenn aufgrund besonderer Umstände Nachteile entstehen können, z.B. wenn der Informationsgehalt dieser Daten für den Betroffenen unter Berücksichtigung seiner psychischen Belange kritisch ist.

Grundsätzlich kommt auch bei Beeinträchtigung schutzwürdiger Interessen die Zulässigkeit der Datennutzung in Betracht, wenn das öffentliche Interesse das Geheimhaltungsinteresse des Betroffenen erheblich überwiegt. Allerdings kann dieser Ausnahmetatbestand nur bei Forschungsvorhaben greifen.

96 Vgl. Bieresborn, in: Hauck/Noftz, SGB X, Stand: 08/2017, § 75 Rn. 6.

97 Vgl. Rombach, in: Hauck/Noftz, SGB X, Stand: 08/2017, § 75 Rn. 22.

98 Rombach, in: Hauck/Noftz, SGB X, Stand: 08/2017, § 75 Rn. 25.

99 Vgl. Rombach, in: Hauck/Noftz, SGB X, Stand: 08/2017, @ 75 Rn. 25.

100 Rombach, in: Hauck/Noftz, SGB X, Stand: 08/2017, § 75 Rn. 28. 
Soweit das öffentliche Interesse an der Planung angesprochen ist, dürfte ein Redaktionsversehen vorliegen, da die Planung im Sozialleistungsbereich durch den Leistungsträger stets im öffentlichen Interesse erfolgt. ${ }^{101}$

\section{Vorrang der Einwilligung (S 75 Abs. I S. 2 SGB X)}

\ 75 Abs. 1S. 2 SGB X bestimmt, dass vor einer Übermittlung eine Einwilligung der betroffenen Person einzuholen ist. Da die Verweisung in $\$ 67 \mathrm{C} \mathrm{Abs.} 2$ Nr. 2 SGB X ausdrücklich „, 75 Absatz 1, 2 oder 4a Satz 1" benennt, ist davon auszugehen, dass mit der Verweisung auf Absatz 1 nicht lediglich Satz 1 des Absatzes gemeint ist, sondern auf alle Regelungen des Absatzes 1 verwiesen wurde. Wäre dies nicht gewollt, wäre die Verweisung - wie in Absatz 4a - auf einen bestimmten Satz beschränkt. Der Vorrang der Einwilligung gilt insofern auch in vorliegender Konstellation. ${ }^{102}$ Hiervon besteht nur dann ein Dispens, wenn die Einholung unzumutbar ist. Die Zumutbarkeit ist dabei nicht bereits deswegen zu verneinen, weil ein nicht unerheblicher Verwaltungsaufwand erforderlich ist, um die Anforderungen der Vorschrift zu erfüllen. ${ }^{103}$

Eine Einwilligung ist gemäß $₫ 75$ Abs. 1 S. 3 SGB X zudem nicht erforderlich, wenn Angaben über den Namen und Vornamen, die Anschrift, die Telefonnummer oder die für die Einleitung eines Vorhabens nach Satz 1 zwingend erforderlichen Strukturmerkmale der betroffenen Person für Befragungen betroffen sind.

\section{Keine Anwendbarkeit des $\$ 67 c$ Abs. 5 SGB X}

Die Vorgaben des $\$ 67$ c Abs. 5 SGB X, wonach Daten, die für Zwecke der Planung im Sozialleistungsbereich erhoben und gespeichert wurden, nur für bestimmte Vorhaben verändert oder genutzt werden dürfen, alsbald anonymisiert und bis dahin getrennt gespeichert werden müssen, ist bereits nach dem Wortlaut in der vorliegenden Konstellation nicht einschlägig. $\$ 67 \mathrm{c} \mathrm{Abs.} 5$ SGB X lautet:

„Für Zwecke der wissenschaftlichen Forschung oder Planung im Sozialleistungsbereich erhobene oder gespeicherte Sozialdaten dürfen von den in $\$ 35$ des Ersten Buches genannten Stellen nur für ein bestimmtes Vorhaben der wissenschaftlichen Forschung im Sozialleistungsbereich oder der Planung im Sozialleistungsbereich verändert oder genutzt werden. Die Sozialdaten sind zu anonymisieren, sobald dies nach dem Forschungs- oder Planungszweck möglich ist. Bis dahin sind die Merkmale gesondert zu speichern, mit denen Einzelangaben über persönliche oder sachliche Verhältnisse einer bestimmten oder bestimmbaren Person zugeordnet werden können. Sie dürfen mit den Einzelangaben nur zusammengeführt werden, soweit der Forschungs- oder Planungszweck dies erfordert.“

101 Vgl. Rombach, in: Hauck/Noftz, SGB X, Stand: 08/2017, \ 75 Rn. $29 f$.

102 Zu den Voraussetzungen der Einwilligung s. Kap. 1.4.3.3.

103 Vgl. Bieresborn, in: Hauck/Noftz, SGB X, Stand: 08/2017, § 75 Rn. 6ff. m.w.N. 
Vorliegend geht es um im Rahmen des $\mathbb{} 284$ Abs. 1 SGB V erhobene und gespeicherte Daten. Sie wurden somit nicht bereits für Zwecke der Planung im Sozialleistungsbereich erhoben und gespeichert. Eine Zweckänderung soll aber derart möglich sein, dass für Zwecke der wissenschaftlichen Forschung oder Planung im Sozialleistungsbereich erhobene und gespeicherte Daten für andere Vorhaben der wissenschaftlichen Forschung oder Planung im Sozialleistungsbereich verwendet werden können. ${ }^{104}$

Missverständlich ist angesichts des klaren Wortlauts die ihr widersprechende Gesetzesbegründung. Demnach soll das geltende Recht zwar beibehalten werden. Dann heißt es aber:

„Demnach können dieverantwortlichen Datenerheber (Erstverarbeiter) die personenbezogenen Daten, die ursprünglich für einen anderen Zweck erhoben wurden, für Forschungszwecke weiterverarbeiten. Die in \$35SGB I genannten Stellen dürfen die Sozialdaten, die sie ursprünglich bei der Erfüllung ihrer gesetzlichen Aufgaben erhoben haben, für Forschungszwecke verändern oder nutzen. Mit der Pflicht zur Anonymisierung von Sozialdaten, sobald dies nach dem Forschungs-oder Planungszweck möglich ist, wird dem informationellen Selbstbestimmungsrecht Rechnung getragen. " 105 [Hervorhebung nicht im Original]

Der Wortlaut einer Vorschrift stellt allerdings die Grenze der Auslegung dar. Somit greift Absatz 5 im vorliegenden Fall, in dem die Daten nicht zu Forschungs- und Planungszwecken erhoben werden, nicht ein. Fraglich ist aber angesichts der unmittelbaren Geltung der DSGVO, ob die Vorgaben des Absatzes 5 nicht trotzdem beachtet werden müssen. In Art. 89 DSGVO sind Anforderungen an die Zulässigkeit zweckändernder Nutzung sensibler Daten enthalten. Erforderlich sind demnach Garantien für die Datensicherheit, mit denen sichergestellt ist, dass technische und organisatorische Maßnahmen bestehen, mit denen insbesondere die Achtung der Datenminimierung gewährleistet wird. Zu diesen Maßnahmen kann die Pseudonymisierung gehö-

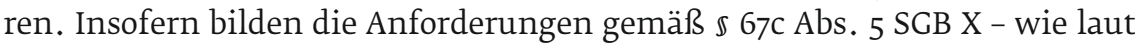
der Gesetzesbegründung beabsichtigt - die Vorgaben des Art. 89 DSGVO ab. In Satz 2 heißt es insbesondere, dass die Sozialdaten zu anonymisieren sind, sobald dies nach dem Forschungs- oder Planungszweck möglich ist.

Allerdings ist der Rückgriff auf die Regelungen $\$$ 67c Abs. 5 SGB X nicht erforderlich. Denn die Vorgaben der DSGVO wurden im Rahmen der hier maßgeblichen Erlaubnisnorm ( $\$ 67 \mathrm{C}$ Abs. 2 Nr. 2 SGB X) bereits durch die Verweisung auf die oben dargestellten Regelungen in $\$ 75$ Abs. 1. SGB X eingehalten.

104 Rombach, in: Hauck/Noftz, SGB X, Stand: 08/2017, § 67c Rn. 61.

105 BT-Drs. 18/12611, S. 114. 


\section{Keine Anwendbarkeit des $\$ 75$ Abs. 2 und Abs. 4a S. 1 SGB X}

Ferner verweist $\$ 67 c$ Abs. 2 Nr. 2 SGB X auf die Voraussetzungen des $\$ 75$ Abs. 2 oder 4a S. 1 SGB X. Durch die Verweisungen auf den Abs. 2 und Abs. 4a S. 1 des $\mathbb{\$} 75$ SGB X soll sichergestellt werden, dass die betroffenen Stellen die in $\mathbb{} 75$ SGB X geregelten neuen Verarbeitungsbefugnisse in Anspruch nehmen können. ${ }^{106}$ Neu geschaffen wurden die Möglichkeiten im Bereich der Forschungsfolgefragen ( $\$ 75$ Abs. 2 SGB X) und hinsichtlich noch nicht bestimmter Forschungsvorhaben des gleichen Forschungsbereichs ( $\$ 75$ Abs. 4a SGB X).

Die Vorschriften betreffen nach ihrem Wortlaut die Nutzung der Daten allein zu Forschungszwecken, Planungszwecke sind hiervon nicht erfasst. Dies ergibt sich hinsichtlich $\mathbb{} 75$ Abs. 2 SGB X auch aus der Gesetzesbegründung, in der es um Datennutzung durch „Forscher bzw. Forschungseinrichtungen“ geht. ${ }^{107}$

Die Gesetzesbegründung zu $\$ 75$ Abs. 4a SGB X ist dagegen widersprüchlich, weil sie einerseits auf den „Bereich der Forschung und Planung“, andererseits nur auf „Forschungsvorhaben“ und nicht auf Planungsvorhaben, abstellt. ${ }^{108}$ Es ist davon auszugehen, dass es sich hierbei um ein Redaktionsversehen handelt und - entsprechend dem Wortlaut - nur hinsichtlich der Datennutzung zu Forschungszwecken erweiterte Möglichkeiten vorgesehen werden sollten. Dies ergibt sich insbesondere auch angesichts des Hintergrundes der Neuregelung in Abs. 4a SGB X, mit der die Wertung des Erw.Gr. 33 der DSGVO aufgegriffen werden soll. ${ }^{109}$ Dort heißt es:

„Oftmals kann zum Zweck der Verarbeitung personenbezogener Daten für Zwecke der wissenschaftlichen Forschung zum Zeitpunkt der Erhebung der personenbezogenen Daten nicht vollständig angegeben werden. Daher sollte es den betroffenen Personen erlaubt sein, ihre Einwilligung für bestimmte Bereiche wissenschaftlicher Forschung zu geben, wenn dies unter Einhaltung der anerkannten ethischen Standards der wissenschaftlichen Forschung geschieht. Die betroffenen Personen sollten die Gelegenheit erhalten, ihre Einwilligung nur für bestimmte Forschungsbereiche oder Teile von Forschungsprojekten in dem vom verfolgten Zweck zugelassenen Maße zu erteilen.“

Diese Ausführungen können auf den Forschungsbereich übertragen werden, was ausweislich der Ausführungen in der Gesetzesbegründung vom Gesetzgeber auch beabsichtigt war.

Für die vorliegend interessierende Auswertung der Daten durch den Leistungsträger zu Planungszwecken kommt es somit auf die Voraussetzungen des $\mathbb{} 75$ Abs. 2 und 4a S. 1 SGB X nicht an.

106 BT-Drs. 18/12611, S. 114.

107 Vgl. BT-Drs. 18/12611, S. 118.

108 BT-Drs. 18/12611, S. 119.

109 Vgl. BT-Drs. 18/12611, S. 119. 
Diese Verweisungen betreffen jeweils Möglichkeiten und Voraussetzungen der Datennutzung zu Forschungszwecken und sind im Übrigen vorliegend auch für die Datennutzung zu Forschungszwecken wegen der vorrangigen Geltung des $\mathbb{2} 287$ SGB V für die (Eigen-)Auswertung der Daten durch den Leistungsträger nicht einschlägig.

\section{Anforderungen an i.R.d. \$ 295 Abs. 1 b S. 1 SGB V übermittelte Daten (S 284 Abs. 3 S. 2 SGB V)}

Darüber hinaus statuiert $\$ 284$ Abs. 3 S. 2 SGB V ${ }^{110}$ eine Pflicht zur Löschung des Versichertenbezugs vor der Auswertung der Daten durch den Leistungsträger, wenn es sich bei den zu übermittelnden Daten um solche handelt, die gemäß $\mathbb{S} 295 \mathrm{Abs}$. 1b S. 1 SGB V von den Leistungserbringern übermittelt wurden. So können versichertenbezogene Abrechnungsdaten, die die Krankenkasse etwa im Zusammenhang mit einem Vertrag zur integrierten Versorgung ohne Beteiligung der kassenärztlichen Vereinigung von den Leistungserbringern er-

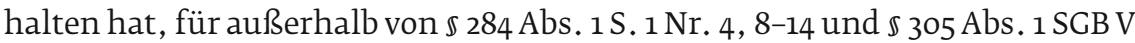
liegende Zwecke - folglich auch für Zwecke der wissenschaftlichen Forschung, der Planung und der Qualitätssicherung - nicht an Dritte übermittelt werden. Vielmehr ist der Versichertenbezug vor einer Übermittlung zu diesen Zwecken zu löschen. Ob der Gesetzgeber hier bewusst auf den Terminus der Anonymisierung verzichtet hat, ist unsicher. Folglich ist bei der Übermittlung der Sozialdaten zu Forschungs- und Planungszwecken i.R.v. $\$ 75$ SGB X zu unterscheiden, ob es sich um Daten i.S.d. $\$ 295$ Abs. 1b S. 1 SGB V handelt oder nicht. Ist Ersteres der Fall, sind die Daten gemäß $\mathbb{2} 284$ Abs. 3 S. 2 Hs. 2 SGB V zwingend vom Versichertenbezug zu befreien.

\section{Vereinbarkeit mit der DSGVO}

Eine Unvereinbarkeit der Erlaubnisnormen ( $\$ 67 \mathrm{~b}$ Abs. 1 S. 1, 2 SGB X i.V.m. $\mathbb{S} 284$ Abs. 3 SGB V, $\mathbb{5} 67 \mathrm{C}$ Abs. 2. Nr. 2 SGB X) mit der DSGVO ist nicht ersichtlich. Die Zulässigkeit einer nationalen Norm zur zweckändernden Verarbeitung von Gesundheitsdaten folgt aus Art. 9 Abs. 2 lit. h) bzw. j) DSGVO. Die Zulässigkeit ist daher zum einen an die Bedingungen und Garantien gem. Art. 9 Abs. 3 (lit. h]) sowie an die Anforderungen des Art. 89 DSGVO (lit. j) geknüpft. Vorliegend dürften die Voraussetzungen des Art. 89 DSGVO durch die Verweisung auf die einschränkenden Regelungen aus $₫ 75$ SCB X der seinerseits auf $\mathbb{2} 2$ Abs. 2 BDSG verweist, erfüllt werden.

Die Datenverarbeitung zu Planungszwecken im Sinne des $\mathbb{s} 67 \mathrm{c}$ Abs. 2 Nr. 2 SGB X dürfte jedoch (nur) unter die Ausnahmevorschrift in Art. 9 Abs. 2

110 \284 Abs. 3 S. 2 SGB V lautet: „Die Daten, die nach \$295 Abs. 1 b Satz 1 an die Krankenkasse übermittelt werden, dürfen nur zu Zwecken nach Absatz 1 Satz 1 Nr. 4, 8, 9, 10, 11, 12, 13, 14 und \$ 305 Abs. 1 versichertenbezogen verarbeitet und genutzt werden und nur, soweit dies für diese Zwecke erforderlich ist; für die Verarbeitung und Nutzung dieser Daten zu anderen Zwecken ist der Versichertenbezug vorher zu löschen." 
lit. h) DSGVO, nicht aber lit. j), fallen. In diesem Fall dürften die Daten gemäß Art. 9 Abs. 3 DSGVO nur verarbeitet werden, wenn diese Daten von Fachpersonal oder unter dessen Verantwortung verarbeitet werden und dieses Fachpersonal dem Berufsgeheimnis unterliegt, oder wenn die Verarbeitung durch eine andere Person erfolgt, die ebenfalls einer Geheimhaltungspflicht unterliegt.

\section{Zwischenergebnis}

Die Auswertung der Sozialdaten durch den Leistungsträger zu Planungszwecken im Sozialleistungsbereich ist nur unter sehr engen Voraussetzungen zulässig. Vorliegend dürfte die Erlaubnisvorschrift insbesondere deswegen in vielen Fällen greifen, weil die Sozialdaten vor der Auswertung anonymisiert werden sollen und das Personal des Leistungsträgers zur Geheimhaltung verpflichtet ist.

\subsection{Nutzung zur Qualitätssicherung (\$ 29g SGB V)}

Die Auswertung der Sozialdaten zur Qualitätssicherung könnte grundsätzlich auf Grundlage des $\mathbb{6} 67$ b Abs. 1, S. 1, 2 SGB X i.V.m. $\mathbb{} 284$ Abs. 3, $\mathbb{} 299$ Abs. 1 a SGB V zulässig sein. Die Krankenkassen sind gemäß $₫ 299$ Abs. 1a SGB V befugt und verpflichtet, nach $\mathbb{2} 284$ Abs. 1 SGB V erhobene und gespeicherte Sozialdaten zu verarbeiten oder zu nutzen, wenn die einzelnen Voraussetzungen der Vorschrift vorliegen.

Neben den in Absatz 1a selbst genannten Voraussetzungen gelten auch die in Absatz 1 Satz 3 bis 7 entsprechend ( $\$ 299$ Abs. 1a S. 3). Gemäß $\$ 299$ Abs. 1 S. 4 Nr. 2 SGB V muss die Auswertung der Daten von einer unabhängigen Stelle vorgenommen werden, wenn sie nicht die Datenauswertung durch die Kassenärztliche Vereinigung im Rahmen der Qualitätsprüfungen betrifft. Die Vorschrift greift insofern bereits deswegen nicht für die hier interessierende Selbstauswertung durch den Leistungsträger.

Dies ergibt sich auch daraus, dass die Auswertung der Daten zu Qualitätssicherungszwecken gemäß $\mathbb{2} 299$ Abs. 1a SGB V in Richtlinien und Beschlüssen des Gemeinsamen Bundesausschusses nach $\mathbb{5} 135 \mathrm{~b}$ Abs. 2 und 136 Abs. 1 S. 1

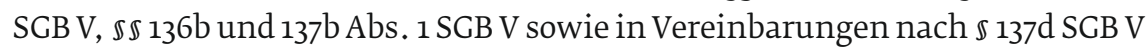
vorgesehen sein muss. Dabei müssen in diesen Richtlinien, Beschlüssen und Vereinbarungen gemäß $\mathbb{2} 299$ Abs. 1a S. 3 SGB V u.a. auch die Empfänger der betroffenen Daten festgelegt werden. Ein Empfänger setzt insoweit einen Übermittlungsprozess und keine Selbstauswertung der Daten voraus. In der Gesetzbegründung heißt es hierzu:

„In Absatzıa werden erstmals die Krankenkassen gesetzlich legitimiert und verpflichtet, bestimmte nach $\$ 284$ Absatz 1 rechtmäßig erhobene und gespeicherte versicherten- und einrichtungsbezogene Daten für die Zwecke der Qualitätssicherung an den 
in den Richtlinien festgelegten Empfänger zu übermitteln. Damit wird die Möglichkeit geschaffen, im Prozess der Qualitätssicherung aufVersichertenstamm- und Abrechnungsdaten der Krankenkassen zurückzugreifen, soweit diese Rückschlüsse auf die Qualität der Leistungserbringung ermöglichen (z.B. Abrechnungsdaten zu Komplikationen einer Behandlung). Dieser Rückgriff steht allerdings unter der Voraussetzung der Erforderlichkeit für die konkreten Maßnahmen der Qualitätssicherung. Eine entsprechende Richtlinienregelung ist zulässig, wenn sich nach Abwägung der maßgeblichen Aspekte wie Nutzen und Informationsgewinn dieser Daten, Ersparnis gesonderter Datenerhebungen bei weiteren Stellen, Reduzierung von Verwaltungsaufwand und Bestehen weniger eingreifender Alternativen der Informationsbeschaffung sich die konkret vorgesehenen Datenübermittlungen der Krankenkassen als vorzugwürdiges Ergebnis erweisen. Entsprechende Erwägungen müssen im Richtlinienbeschluss zum Ausdruck kommen." "w [Hervorhebung nicht im Original]

Aus den Bestimmungen zur Festlegung des Empfängers und der Gesetzesbegründung ergibt sich letztlich auch, dass es sich bei $\mathbb{} 299$ Abs. 1a SGB V um eine weitere Erlaubnis- bzw. Verpflichtungsvorschrift hinsichtlich der Übermittlung der Daten durch die Leistungsträger zum Zwecke der Qualitätssicherung an einen Dritten (eine unabhängige Stelle) handelt. Die Selbstauswertung sollte nicht Inhalt der neu geschaffenen Möglichkeiten sein.

Die Selbstauswertung durch den Leistungsträger zum Zwecke der Qualitätssicherung gemäß $\$ 299$ Abs. 1a SGB V scheidet insofern aus. Auch anderen Erlaubnisvorschriften sind insoweit nicht ersichtlich.

\subsubsection{Einwilligung}

Neben einer gesetzlichen Ermächtigung kann sich die Zulässigkeit der Verarbeitung aus einer Einwilligung des Betroffenen ergeben (Art. 6 Abs. 1 lit. a] DSGVO, Art. 9 Abs. 2 lit. a] DSGVO), die bei allen hier relevanten Zwecken in Betracht kommt. Während für Einwilligungen nach Art. 6 Abs. 1 lit. a) DSGVO kein Regelungsspielraum der Mitgliedsstaaten verbleibt, da hierfür keine Öffnungsklausel in der DSGVO eingreift, ergibt sich ein solcher für den Bereich der Verarbeitung besonderer Kategorien personenbezogener Daten (im Folgenden auch: sensible Daten) aus der Öffnungsklausel des Art. 9 Abs. 2 lit. a) DSGVO. ${ }^{112}$ Diesbezüglich kann sich aus dem Unionsrecht oder dem Recht der Mitgliedsstaaten ergeben, dass das grundsätzliche Verbot der Verarbeitung etwa von Gesundheitsdaten durch eine Einwilligung nicht aufgehoben werden kann. Wenn also eine nationale Vorschrift existiert, die die Einwilligungsmöglichkeit ausschließt, ist die Datenverarbeitung gemäß Art. 9 Abs. 1 DSGVO verboten. Wenn den Mitgliedsstaaten die Regelungsbefugnis zukommt, die Einwilligung vollständig auszuschließen, bedeutet dies allerdings auch, dass

111 BT-Drs. 17/8005, S. 130.

112 Kühling/Martini et al., Die DSGVO und das nationale Recht, 2016, S. $316 f$. 
das nationale Recht anstelle eines vollständigen Ausschlusses lediglich engere Voraussetzungen an die Einwilligung, als Minus zum möglichen, vollständigen Ausschluss der Einwilligung, vorgeben kann, als sie in der DSGVO bestimmt sind. ${ }^{1{ }^{13}}$ Der deutsche Gesetzgeber hat für den hier einschlägigen Bereich zunächst hinsichtlich nicht sensibler Sozialdaten die Einwilligungsmöglichkeit in $\$ 67 \mathrm{~b}$ Abs. 1 S. 1 SGB X nicht mehr aufgeführt und hinsichtlich der Einwilligung in die Verarbeitung von sensiblen Daten von seiner Regelungskompetenz zum vollständigen Ausschluss der Einwilligung keinen Gebrauch gemacht (vgl. $\mathbb{5} 67 \mathrm{~b}$ Abs. 1 S. 2, 3 SCB X). ${ }^{114}$ Dementsprechend ist eine Einwilligung grundsätzlich auch für diesen Bereich möglich.

Die grundlegenden Anforderungen an die Einwilligung sind in Art. 4 Nr. 11 und Art. 7 DSGVO festgelegt. Gemäß Art. 4 Nr. 11 DSGVO ist eine Einwilligung

,jede freiwillig für den bestimmten Fall, in informierter Weise und unmissverständlich abgegebene Willensbekundung in Form einer Erklärung oder einer sonstigen eindeutigen bestätigenden Handlung, mit der die betroffene Person zu versehen gibt, dass sie mit der Verarbeitung der sie betreffenden personenbezogenen Daten einverstanden ist.“

Art. 7 DSGVO ergänzt die Anforderungen dahingehend, dass die Einwilligung jederzeit widerruflich (Abs. 3) und der Verantwortliche zum Nachweis der Einwilligungserklärung verpflichtet ist (Abs. 1). Im Bereich sensibler Daten ergeben sich aus Art. 9 Abs. 2 lit. a) DSGVO darüber hinaus noch weitergehende Anforderungen an eine Einwilligung. Auch nationale sozialdatenschutzrechtliche Regelungen enthalten zusätzliche Anforderungen an eine wirksame Einwilligung als Grundlage für die Datenverarbeitung. Im Einzelnen:

\subsection{Freiwilligkeit}

Bereits nach der Definition der Einwilligung nach Art. 4 Nr. 11 DSGVO ist die Freiwilligkeit der Einwilligung erforderlich. Besonderes Augenmerk ist bei der Einholung einer Einwilligung im Zusammenhang mit der Datenverarbeitung durch Sozialleistungsträger auf die Gestaltung der Einwilligungserklärungen zu legen. Auf Grund des Abhängigkeitsverhältnisses zwischen Betroffenem und Leistungsträger bewegt sich die Freiwilligkeit der Erklärung stets auf einem schmalen Grat. Dies macht insbesondere Erw.Gr. 43 der DSGVO deutlich. Danach

„sollte diese [die Einwilligung] in besonderen Fällen, wenn zwischen der betroffenen Person und dem Verantwortlichen ein klares Ungleichgewicht besteht, insbesondere

113 Siehe auch: Kühling/Martini et al., Die DSGVO und das nationale Recht, 2016, S. $49 f$.

114 An dieser Stelle sei darauf hingewiesen, dass mit dem Entwurf zum 2. DSAnpUG-EU bei der Verarbeitung von biometrischen, genetischen oder Gesundheitsdaten formelle Wirksamkeitsvoraussetzungen für Einwilligungserklärungen geschaffen werden sollen, vgl. BT-Drs. 19/4674, S. 153. Hierzu bereits oben in Kap. 1.1.3.2.1. 
wenn es sich bei dem Verantwortlichen um eine Behörde handelt, und es deshalb in Anbetracht aller Umstände in dem speziellen Fall unwahrscheinlich ist, dass die Einwilligung freiwillig gegeben wurde, keine gültige Rechtsgrundlage liefern. Die Einwilligung gilt nicht als freiwillig erteilt, wenn zu verschiedenen Verarbeitungsvorgängen von personenbezogenen Daten nicht gesondert eine Einwilligung erteilt werden kann, obwohl dies im Einzelfall angebracht ist, oder wenn die Erfüllung eines Vertrags, einschließlich der Erbringung einer Dienstleistung, von der Einwilligung abhängig ist, obwohl diese Einwilligung für die Erfüllung nicht erforderlich ist."

Dementsprechend ist die Einwilligung derart zu gestalten, dass dem Betroffenen die Wahlmöglichkeit klar vor Augen geführt und betont wird, dass die Nichterteilung keine nachteiligen Folgen für das Versicherungsverhältnis hat und haben wird. Dabei kann die Freiwilligkeit, sofern im Rahmen des konkreten Vorhabens möglich, über ein abgestuftes Einwilligungssystem besonders herausgestellt werden.

Insbesondere ist gemäß Art. 7 Abs. 4 DSGVO zu vermeiden, den Abschluss eines Vertrages an die Abgabe der Einwilligung zu koppeln.

\subsection{Informiertheit/Hinweispflicht}

Grundvoraussetzung der wirksamen Einwilligung ist die Informiertheit des Einwilligenden. Der Betroffene ist vorab über die wesentlichen Punkte der Datenverarbeitung, wie den Verantwortlichen den Empfänger, die Übermittlungswege und -zwecke sowie das Forschungsprojekt zu informieren. ${ }^{15}$ Erw. Gr. 42 der DSGVO konkretisiert die Informationspflichten des Verantwortlichen dahingehend, dass der Betroffene über den Verantwortlichen und die Zwecke der Datenverarbeitung aufzuklären ist und dass er erkennen kann, dass und in welchem Umfang er seine Einwilligung erteilt. Gemäß $₫ 67 \mathrm{~b}$ Abs. 2 S. 2 SGB X muss die betreffende Person auf den Zweck der vorgesehenen Verarbeitung, auf die Folgen der Verweigerung der Einwilligung sowie auf die jederzeitige Widerrufsmöglichkeit im Rahmen der Einholung der Einwilligung hingewiesen werden. Der deutliche Hinweis auf diese Punkte ist dabei auch von erheblicher Bedeutung für die Freiwilligkeit der Erklärung, denn auf Grund des bestehenden Abhängigkeitsverhältnisses der Betroffenen von den Leistungsträgern kann die Freiwilligkeit der Einwilligung nur bejaht werden, wenn dem Betroffenen klar wird, dass die Erteilung einer Einwilligung für ihn nicht zwingend ist. Hierdurch wird insbesondere auch der Forderung im Erw.Gr. 32 der DSGVO entsprochen, wonach die Einwilligung freiwillig, für den konkreten Fall und in informierter Weise erfolgen soll. Damit die betreffende Person die Einwilligung in Kenntnis der Sachlage abgeben kann, muss sie wiederum wissen, für welche Zwecke ihre Daten verarbeitet werden sollen (vgl. Erw.Gr. 42 DSGVO).

115 Rombach, in: Hauck/Noftz, Stand: 08/2017, SGB X, § 75 Rn. 32. 
Mangels einer konkretisierenden Regelung in der DSGVO, welche Informationen der Einwilligende genau erhalten soll, kann der nationale Gesetzgeber entsprechende Vorgaben machen.

\subsection{Zweckbindung/Umfang}

Bereits aus der Definition in Art. 4 Nr. 11 DSGVO ergibt sich, dass die Einwilligung nur für einen bestimmten Fall abgegeben werden kann. Art. 6 Abs. 1 S. 1 lit. a) DSGVO sowie Art. 9 Abs. 2 lit. a) DSGVO konkretisieren dieses Erfordernis dahingehend, dass sich die Einwilligung auf einen oder mehrere festgelegte Zwecke beziehen muss. Das Maß der Konkretisierung hängt dabei regelmäßig von der geplanten Verwendungssituation ab. ${ }^{116}$ Dementsprechend ist das konkrete Vorhaben im Rahmen der Einwilligung zu benennen und soweit möglich zu beschreiben.

Bei der Verarbeitung von Daten zu Forschungszwecken gilt darüber hinaus $\$ 67$ Abs. 3 SGB X, wonach die Einwilligung für ein bestimmtes Vorhaben oder für bestimmte Bereiche der wissenschaftlichen Forschung erteilt werden kann (Broad Consent). Mit dieser erweiterten Einwilligung soll den Bedürfnissen der Wissenschaft Rechnung getragen werden, wenn etwa zum Zeitpunkt der Erhebung der Sozialdaten noch nicht das konkrete Forschungsvorhaben, jedoch bestimmte Forschungsbereiche angegeben werden können. Durch die Regelung sollte berücksichtigt werden, dass Forschungsfragen teilweise in einer offenen Vorgehensweise sukzessive entwickelt werden ${ }^{117}$. Dies greift den Gedanken des Erw.Gr. 33 DSGVO auf, der das Konzept einer solchen „weiten Einwilligung “ für die DSGVO beinhaltet, ohne dass der deutsche Gesetzgeber aber gleichzeitig die Bezugnahme auf die zur Kompensation von Erw.Gr. 33 geforderte „Einhaltung der anerkannten ethischen Standards der wissenschaftlichen Forschung“ ausdrücklich aufgreift. ${ }^{118}$ Das führt nach hier vertretener Auffassung aber nicht zur Unvereinbarkeit mit der DSGVO, da die Erwägungsgründe zum einen kein verbindlicher Verordnungstext sind und überdies auch Erw.Gr. 33 nicht fordert, dass ein Mitgliedsstaat die Einhaltung ethischer Standards im jeweiligen Datenschutzrecht normiert. Verpflichtungen zur Einhaltung ergeben sich aus diversen Rechtsquellen außerhalb des nationalen Datenschutzrechts. ${ }^{119}$

Um dem Grundsatz der informierten Einwilligung Rechnung zu tragen, sollte der in der Einwilligung zu nennende Bereich der Forschung nicht zu allgemein gefasst sein und ist thematisch möglichst einzugrenzen. Die Teilnehmer wissenschaftlicher Studien sollten zudem über das Vorgehen informiert und darauf hingewiesen werden, dass sich die Forschungsfragen in einem

116 Buchner/Kühling, in: Kühling/Buchner, DS-GV0, 2017, Art. 7 Rn. 65.

117 BT-Drs. 18/12611, S. 113.

118 Siehe zum Broad Consent auch: Kap. 3.2.

119 Siehe hierzu Kap. 3.2.6. 
abgegrenzten thematischen Feld bewegen, jedoch schrittweise konkretisiert werden. ${ }^{120}$

\subsection{Form und Nachweispflicht}

Hinsichtlich der Form der Einwilligung bestehen weder nach den SGB X noch der DSGVO zwingende Vorschriften. $\$ 67 \mathrm{~b}$ Abs. 2 S. 1 SGB X regelt, dass die Einwilligung nach Möglichkeit schriftlich oder elektronisch eingeholt werden soll. Diese Soll-Vorschrift dient im Wesentlichen der Sicherstellung der Nachweispflicht (Art. 7 Abs. 1 DSGVO), steht aber einer anderweitigen Form - etwa der mündlich erteilten, protokollierten Einwilligung - im Falle besonderer Umstände nicht entgegen. ${ }^{121}$ Dennoch ist es ratsam, die Einwilligungen zu den hier beabsichtigten Zwecken schriftlich oder hilfsweise elektronisch einzuholen. Mit der Möglichkeit einer elektronischen Erklärung soll berücksichtigt werden, dass in Zukunft immer mehr Verwaltungsverfahren elektronisch geführt werden. Zur Vermeidung von Medienbrüchen soll durch die ergänzende Möglichkeit der elektronischen Erklärung diesen Entwicklungen Rechnung getragen werden. ${ }^{122}$ Nicht ausreichend ist dagegen Stillschweigen, bereits angekreuzte Kästchen oder Untätigkeit der betroffenen Person. Durch die vorgeschriebene Nachweispflicht wird die Beweislast für das Vorliegen einer wirksamen Einwilligung dem Leistungsträger auferlegt. Letztlich laufen diese Vorgaben auf eine Dokumentationspflicht für den gesamten Verarbeitungsvorgang und die Einholung der jeweiligen Einwilligung hinaus. ${ }^{123}$

Sind von der Datenverarbeitung besondere Kategorien personenbezogener Daten umfasst, regelt Art. 9 Abs. 2 lit. a) DSGVO zudem, dass die Einwilligung ausdrücklich erfolgen muss. Eine mutmaßliche Einwilligung kommt ebenso wie eine konkludent durch schlüssiges Verhalten geäußerte nicht in Betracht. ${ }^{124}$ Die Ausdrücklichkeit der Einwilligung gemäß Art. 9 Abs. 2 lit. a) DSGVO soll den Betroffenen auf die besondere Sensibilität der Daten aufmerksam machen. Konkret wird gefordert, dass der betroffenen Person unter konkreter Nennung der Datenkategorie verdeutlicht werden muss, dass die entsprechenden Daten von der Einwilligung erfasst sind. ${ }^{125}$ Auch unter diesem Gesichtspunkt ist eine schriftliche oder elektronische Einwilligung folglich empfehlenswert.

Es sei an dieser Stelle auf den eingangs erwähnten Gesetzentwurf zum 2. DSAnpUG-EU hingewiesen, wonach strengere Wirksamkeitsvoraussetzungen an die datenschutzrechtliche Einwilligungserklärung bei der Verarbeitung

\footnotetext{
120 BT-Drs. 18/12611, S. 113.

121 BT-Drs. 18/12611, S. 113.

122 BT-Drs. 18/12611, S. $112 f$.

123 Vgl. Plath, in: Plath, BDSG/DSGV0, 2. Aufl., 2016, Art. 7 Rn. 3.

124 Frenzel, in: Paal/Pauly, Datenschutz-Grundverordnung, 2017, Art. 9 Rn. 21; Schiff, in: Ehmann/Selmayr, DS-GVO, 2017, Art. 9 Rn. 28.

125 Plath, in: Plath, BDSG/DSGV0, 2. Aufl., 2016, Art. 9, Rn. 13.
} 
von genetischen, biometrischen und Gesundheitsdaten in einem neuen $\mathbb{6} 6 \mathrm{~b}$ Abs. 2 S. 2, Abs. 3 SGB X geplant sind. ${ }^{126}$

\subsection{Hervorhebungsgebot}

In Art. 7 Abs. 2 DSGVO werden für den Fall, dass die Einwilligung schriftlich erfolgt und noch andere Sachverhalte betrifft, weitere Voraussetzungen vorgegeben. Diese muss dann in verständlicher und leicht zugänglicher Form in einer klaren und einfachen Sprache so erfolgen, dass es von den anderen Sachverhalten klar zu unterscheiden ist. Es gilt somit das sog. Trennungs- und Transparenzprinzip, insbesondere auch zur Verhinderung „versteckter“ Einwilligungserklärungen in den Allgemeinen Geschäftsbedingungen.

\subsection{Widerrufsmöglichkeit}

Gemäß Art. 7 Abs. 3 DSGVO hat die betroffene Person jederzeit das Recht, ihre Einwilligung zu widerrufen. Der Widerruf muss so einfach wie die Erteilung der Einwilligung sein. Hierdurch kann der Betroffene seine Einwilligung ex nunc beseitigen und zukünftige Datenverarbeitung auf Grundlage seiner Einwilligung verhindern. ${ }^{127}$

\subsection{Zwischenergebnis}

Unter Beachtung der aufgezeigten Anforderungen kommt die Auswertung der Sozialdaten jeweils zu Forschungs-, Planungs- sowie Qualitätssicherungszwecken in Betracht.

Im Bereich der Datenauswertung zu Forschungszwecken gilt insofern eine Erleichterung hinsichtlich der Zweckbindung, als die Nennung bestimmter Bereiche der Forschung zulässig ist, soweit eine weitere Konkretisierung zum Zeitpunkt der Einwilligung noch nicht möglich ist.

\subsubsection{Angemessene, spezifische Maßnahmen (\$ 22 Abs. 2 BDSG)}

Bei der Auswertung der Sozialdaten durch den Leistungsträger handelt es sich auch um die Veränderung oder Nutzung der Sozialdaten im Sinne des $\mathbb{S} 67 \mathrm{~b}$ SGB X. Gemäß $\$$ 67b Abs. 1 S. 4 SGB X gilt $\$ 22$ Abs. 2 BDSG entsprechend und ist auch vorliegend anzuwenden. ${ }^{128} \mathbb{S} 22$ Abs. 2 BDSG lautet wie folgt:

„In den Fällen des Absatzes 1 sind angemessene und spezifische Maßnahmen zur Wahrung der Interessen der betroffenen Person vorzusehen. Unter Berücksichtigung des

126 Hierzu bereits eingangs in Kap. 1.1.3.2.1.

127 Vgl. Plath, in: Plath, BDSG/DSGV0, 2. Aufl., 2016, Art. 7, Rn. 10, für den Bereich der Forschung Schaar, ZD 2017, $213(214,216)$.

128 Vgl. BT-Drs. 18/12611, S. 114. 
Standes der Technik, der Implementierungskosten und der Art, des Umfangs, der Umstände und der Zwecke der Verarbeitung sowie der unterschiedlichen Eintrittswahrscheinlichkeit und Schwere der mit der Verarbeitung verbundenen Risiken für die Rechte und Freiheiten natürlicher Personen können dazu insbesondere gehören:

1. technisch organisatorische Maßnahmen, um sicherzustellen, dass die Verarbeitung gemäßder [DSGVO] erfolgt,

2. Maßnahmen, die gewährleisten, dass nachträglich überprüft und festgestellt werden kann, ob und von wem personenbezogene Daten eingegeben verändert oder entfernt worden sind,

3. Sensibilisierung der an Verarbeitungsvorgängen Beteiligten,

4. Benennung einer oder eines Datenschutzbeauftragten,

5. Beschränkung des Zugangs zu den personenbezogenen Daten innerhalb der verantwortlichen Stelle und von Auftragsverarbeitern,

6. Pseudonymisierung personenbezogener Daten,

7. Verschlüsselung personenbezogener Daten,

8. Sicherstellung der Fähigkeit, Vertraulichkeit, Integrität, Verfügbarkeit und Belastbarkeit der Systeme und Dienste im Zusammenhang mit der Verarbeitung personenbezogener Daten, einschließlich der Fähigkeit, die Verfügbarkeit und den Zugang bei einem physischen oder technischen Zwischenfall rasch wiederherzustellen,

9. zur Gewährleistung der Sicherheit der Verarbeitung die Einrichtung eines Verfahrens zu regelmäßigen Überprüfung, Bewertung und Evaluierung der Wirksamkeit der technischen und organisatorischen Maßnahmen oder

10. spezifische Verfahrensregelungen, die im Fall einer Übermittlung oder Verarbeitung für andere Zwecke die Einhaltung der Vorgaben dieses Gesetztes sowie der [DSGVO] sicherstellen."

Durch diese Regelung wurde das Erfordernis aus Art. 9 Abs. 2 lit. b), g), i) und j) der DSGVO umgesetzt, geeignete Garantien für die Grundrechte und die Interessen der betroffenen Person bzw. angemessene und spezifische Maßnahmen zur Wahrung der Grundrechte und Interessen der betroffenen Person vorzusehen. ${ }^{129}$

Die Anforderungen sind in $\$ 22$ Abs. 2 BDSG bereits detailliert wiedergegeben. Wie diese in der Praxis im Einzelnen umzusetzen sind, muss im Einzelfall geprüft werden und kann nur anhand eines konkreten Datenschutz-Konzeptes beurteilt werden. 


\subsubsection{Genehmigungserfordernis und -anforderungen}

\subsubsection{Forschungszwecke}

Ein Genehmigungserfordernis besteht bei Auswertung der Daten zu Forschungszwecken ohne Einwilligung der Betroffenen auf Grundlage des $\$ 287$ SGB V. Hier muss die Aufsichtsbehörde dem Leistungsträger eine Erlaubnis erteilen. Das Erlaubnisverfahren ist im Gesetz nicht geregelt. Nach dem Gesetzeszweck ist die Erlaubnis vor Beginn der Forschungsvorhaben erforderlich. Hierdurch soll die Einhaltung der Datenschutzvorschriften gewährleistet werden, sodass die Aufsichtsbehörde über die Einzelheiten des geplanten Forschungsvorhabens (vorher) zu unterrichten ist. ${ }^{130}$

\subsubsection{Planungsvorhaben}

Bei der Auswertung der Daten zu Planungszwecken ohne Einwilligung der Betroffenen auf Grundlage des $\mathbb{5} 67$ Abs. 2 Nr. 2 SGB X ist mangels Verweis auf $\$ 75$ Abs. 4 SGB X kein Genehmigungsverfahren erforderlich. Dennoch ist aufgrund des Verweises auf $\mathbb{S} 75$ Abs. 1 SGB X der zuständigen Aufsichtsbehörde ein Datenschutzkonzept vorzulegen, vgl. $\$ 75$ Abs. 1 S. 4 SGB X. In dem Datenschutzkonzept ist insbesondere darzulegen, dass die technischen und organisatorischen Anforderungen sowie der Grundsatz der Datenminimierung erfüllt sind. ${ }^{131}$ Diese kann bei einer Prüfung sodann im Rahmen ihrer aufsichtsrechtlichen Möglichkeiten auf den Leistungsträger einwirken.

\subsubsection{Ergebnis}

Zu Szenario 1 lassen sich damit folgende Ergebnisse zusammenfassen:

Eine Verarbeitung von Sozialdaten zu Forschungszwecken ist in den Grenzen des \$287 SGB V zulässig. Die Forschungsvorhaben sind dabei nicht auf medizinische Fragestellungen begrenzt. Die Beteiligung einer externen Stelle, die sich darauf beschränkt die Forschungsfrage sowie die Planung des Forschungsvorhabens mitzugestalten, ist unproblematisch. Ebenfalls denkbar wäre eine Einbeziehung einer externen Stelle im Rahmen einer Auftragsverarbeitung. Auswertungen dürfen jedoch nicht von einem Dritten vorgenommen werden. Die Anforderung des $\mathbb{2} 287$ Abs. 2 SGB V, wonach eine Anonymisierung der Daten erforderlich ist, kann nur sinnvoll in der Praxis umgesetzt werden, wenn ein modifiziertes Verständnis des Begriffs der Anonymisierung zugrunde gelegt wird. Hierbei könnte man sich an den Kategorien der sogenannten „formalen Anonymisierung“, wie sie in $\$ 16$ Abs. 2 S. 1 Bundesstatistikgesetz verwendet wird, orientieren. Eine Unvereinbarkeit des $\mathbb{2} 287$ SGB V mit der DSGVO liegt unseres Erachtens nicht

130 Didong, in: jurisPK-SGB V, 3. Aufl., 2016, $\$ 287$ Rn. 11 m.w.N.

131 BT-Drs. 18/12611, S. 118. 
vor. Ein Rückgriff auf den allgemeineren Tatbestand des $\$ 76 \mathrm{c}$ SGB X ist nach vorherrschender Meinung ausgeschlossen. Dem Gesetzgeber stünde es offen, diesen Rückgriff auch unter Beachtung der DSGVO zu ermöglichen.

Eine Nutzung von Sozialdaten durch eine Krankenkasse zu Planungszwecken ist auf Grundlage des gesetzlichen Tatbestandes des $\mathbb{5} 67 \mathrm{C}$ Abs. 2 Nr. 2 SGB X zulässig. Ob eine Anwendung von $\mathbb{5} 75$ Abs. 2 und Absatz 4 S. 1 SCB X in Betracht kommt, ist allerdings fraglich. Die besseren Argumente sprechen dafür, dass die sich aus dem Wortlaut ergebende Begrenzung auf Forschungsvorhaben eine Anwendung für Planungsvorhaben nicht zulässt.

Hinsichtlich einer Nutzung zu Qualitätssicherungszwecken ist eine Auswertung durch die Krankenkasse nach den Vorgaben des $\$ 299$ SGB V nicht zulässig.

Alternativ zu einer gesetzlichen Ermächtigungsnorm kann sowohl ein Forschungsvorhaben, ein Planungsvorhaben als auch ein Qualitätssicherungsvorhaben auf eine Einwilligung gestützt werden. Da der Gesetzgeber von seiner Regelungskompetenz zum vollständigen Ausschluss der Einwilligung im Bereich der Verarbeitung von besonderen Kategorien personenbezogener Daten keinen Gebrauch gemacht hat, ist eine Einwilligung unter Rückgriff auf die DSGVO stets zulässig. Im Rahmen einer denkbaren Anpassung des SGB V an die DSGVO könnte der deutsche Gesetzgeber jedoch die Möglichkeit der Einholung einer Einwilligungserklärung für besondere Kategorien personenbezogener Daten ausschließen oder nur unter erhöhten Anforderungen zulassen.

Bei allen Verarbeitungen sind angemessene, spezifische Maßnahmen zur Wahrung der Interessen der betroffenen Personen vorzusehen, wie sie sich aus der DSGVO bzw. dem nationalen Datenschutzrecht ergeben. Ein Genehmigungserfordernis besteht ausschließlich bei einer Verarbeitung von Sozialdaten zu Forschungszwecken.

\subsection{Beurteilung von Szenario 2}

\subsubsection{Darstellung des Szenarios}

Ein Leistungsträger der gesetzlichen Krankenversicherung und eine externe Einrichtung kooperieren. Die Sozialdaten werden für ein bestimmtes Vorhaben an die externe Einrichtung übermittelt, die diese im Sinne des Vorhabens verarbeitet und auswertet.

\subsubsection{Rechtfertigungsbedürftige Datenverarbeitungsvorgänge}

Bereits vorab wurde klargestellt, dass die Verarbeitung anonymisierter Daten aus datenschutzrechtlicher Sicht nicht relevant ist, sodass auch eine Weitergabe anonymisierter Sozialdaten für ein bestimmtes Vorhaben an eine externe Einrichtung sowie die Verarbeitung und Auswertung durch diese zulässig ist. 
Für pseudonymisierte Sozialdaten gilt Gleiches, wenn nach dem maßgeblichen Verständnis eines relativen Personenbezugs die pseudonymisierten Daten aus Sicht des Empfängers faktisch anonym sind. Dies wäre dann nicht anzunehmen, wenn ein rechtlich zulässiges Mittel bestünde, das eine De-Pseudonymisierung und damit eine Re-Identifikation durch die externe Einrichtung ermöglichte. Ein solches Mittel ist im Forschungs- oder Planungskontext nicht ersichtlich.

Vorliegend kommen daher folgende rechtfertigungsbedürftige Verarbeitungsvorgänge in Betracht: Abhängig von der rechtlichen Ausgestaltung ist zunächst eine Übermittlung (s. Kap. 1.4.3.2) an die externe Stelle zu untersuchen, die diese Daten dann im Sinne einer Auswertung nutzen würde (s. Kap. 1.5.4).

Soll die externe Stelle hingegen als Auftragsverarbeiter tätig werden, stellen sich die Weitergabe der Daten an den Auftragsverarbeiter und die Auswertung durch diesen für den Auftraggeber jeweils als Nutzung durch den verantwortlichen Auftraggeber dar (s. Kap. 1.5.4.4).

\subsubsection{Zulässigkeit der Weitergabe von Sozialdaten durch den Leistungsträger (hinsichtlich der jeweiligen Zwecke) an eine externe Einrichtung}

Sollen personenbezogene Abrechnungsdaten von Versicherten zum Zweck der Verarbeitung für ein bestimmtes Projekt im Bereich der wissenschaftlichen Forschung, Planung im Sozialleistungsbereich oder der Qualitätssicherung an eine externe Einrichtung übertragen werden, bedarf es entsprechend dem generellen Verbot mit Zulässigkeitsvorbehalt einer Ermächtigungsgrundlage. Neben den gesetzlichen Erlaubnistatbeständen (s. Kap. 1.5.3.2) kommt dabei auch die Einwilligung des Betroffenen (s. Kap. 1.5.3.3) in Betracht. Verzichtbar kann eine solche Ermächtigungsgrundlage gegebenenfalls sein, wenn die Daten nicht an einen eigenständigen Dritten, sondern einen weisungsgebundenen Auftragsverarbeiter weitergegeben werden (s. Kap. 1.5.3.4).

\subsubsection{Prüfungsmaßstab/Identifikation des einschlägigen Datenschutzregimes}

Hinsichtlich der Verarbeitungen durch die Krankenkasse bleibt es bei den in Kapitel 1.4.3.1 beschriebenen Rahmenbedingungen.

\subsubsection{Gesetzliche Ermächtigung}

Aufgrund des Vorrangs der bereichsspezifischen Datenschutzregelungen des Sozialgesetzbuchs ${ }^{132}$ bedarf es einer entsprechenden Ermächtigungsgrundlage im SGB. \$ 67b Abs. 1 SGB X bestimmt, dass die Übermittlung von Sozialdaten, inklusive der besonderen Kategorien personenbezogener Daten, durch die Leistungsträger zulässig ist, sofern die Folgevorschriften des SGB X oder eine

132 Vgl. dazu oben Kap. 1.4.3.1. 
andere Rechtsvorschrift des Sozialgesetzbuchs dies erlaubt oder anordnet. Dabei ist zunächst auf die bereichsspezifischen Vorschriften des SGB V abzustellen, bevor eine Ermächtigungsgrundlage des allgemeinen Sozialrechts nach dem SGB X herangezogen wird.

Die $\mathbb{N} 284 \mathrm{ff}$. SGB V regeln bereichsspezifisch die zulässige Verarbeitung von Sozialdaten insbesondere auch durch die Krankenkassen. Dabei gibt $\mathbb{2} 28$ Abs. I S. 1 SGB V abschließend vor, zu welchen Zwecken die Krankenkassen Sozialdaten erheben und speichern dürfen. Für die Verarbeitung (und Nutzung) dieser Daten regelt $\mathbb{\$} 284$ Abs. 3 S. 1 Hs. 1 SGB V, dass die so erhobenen Sozialdaten grundsätzlich nur für die in Absatz 1 genannten Zwecke verarbeitet werden dürfen. Eine Verarbeitung (und Nutzung) dieser Daten für andere

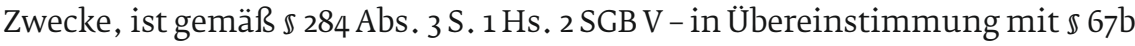
Abs. 1 SGB X - nur zulässig, sofern eine andere Rechtsvorschrift des Sozialgesetzbuchs dies anordnet oder erlaubt. Folglich bedarf die Übermittlung von Daten durch die Leistungserbringer zu Zwecken der wissenschaftlichen Forschung, Planung im Sozialleistungsbereich oder der Qualitätssicherung einer gesonderten Ermächtigungsgrundlage im Sozialgesetzbuch. Wie bereits unter Teil 2 A.III.1.b) (s. Kap. 1.4.3.1.2) dargelegt, geht dabei keine Sperrwirkung von den bereichsspezifischen Erlaubnistatbeständen des SGB V gegenüber dem SCB X aus. Vielmehr enthält das SGB V lediglich spezifischere Vorschriften, die im Einzelfall vorrangig anzuwenden sind, die jedoch darüber hinaus einen Rückgriff auf die allgemeinen Vorschriften nicht gänzlich verwehren.

\subsection{Nutzung zu Forschungszwecken (\$ 287 SGB V)}

In Betracht käme zunächst die bereichsspezifische Regelung des $\mathbb{2} 287$ SGB V. Nach dieser dürfen Krankenkassen mit Erlaubnis der Aufsichtsbehörde die Datenbestände leistungserbringer- oder fallbeziehbar für zeitlich befristete und im Umfang begrenzte Forschungsvorhaben auswerten und aufbewahren. $\mathbb{S} 287$ SGB V ist jedoch ausschließlich auf die krankenkasseninterne Auswertung von leistungserbringer- oder fallbeziehbaren Daten zugeschnitten. ${ }^{133}$ Die Ermächtigung umfasst weder die interne Verarbeitung von versichertenbeziehbaren Daten ${ }^{134}$ noch die Übermittlung der Daten an externe Stellen zur Verarbeitung der Daten zu den beabsichtigten Zwecken. $\$ 287$ SGB V stellt folglich keine geeignete Ermächtigungsgrundlage dar.

\subsection{Nutzung zur Qualitätssicherung (\$ 299 SGB V)}

Cemäß $\mathbb{2} 299$ Abs. 1a SCB V sind Krankenkassen befugt und verpflichtet, die im Rahmen ihrer Aufgabenerfüllung nach $\mathbb{2} 284$ Abs. 1 SGB V erhobenen Sozialdaten für Zwecke der Qualitätssicherung zu verarbeiten und zu nutzen.

133 Vgl. Ausführungen zu Szenario 1 Kap. 1.4.3.2.1, Abschnitt „Interne Fragestellungen“ und Kap. 1.4.3.2.2.

134 Vgl. Didong, in: Schlegel/Voelzke, jurisPK-SGB V, 3. Aufl., 2016, § 287 Rn. 8. 
Der Gemeinsame Bundesausschuss nach $\mathbb{5} 91$ SGB V (G-BA) regelt in Beschlüssen und Richtlinien, welche konkreten Daten für die Verarbeitung und Nutzung zugelassen sind und legt die Empfänger fest. Die von der Krankenkasse zu übermittelnden Sozialdaten sind in Anlage II Teil b) der Richtlinie zur einrichtungsund sektorenübergreifenden Qualitätssicherung (Quesü-RL) ${ }^{135}$ festgeschrieben. Dazu gehören beispielsweise die Stammdaten der Versicherten. Gemäß $\mathbb{S}$ Abs. 1 Satz 9, Teil 1 Quesü-RL haben die Krankenkassen die Daten an die vom G-BA nach $\mathbb{} 4$ Abs. 5 Satz 1, Teil 1 Quesü-RL beauftragte Datenannahmestelle (DAS-KK) zu übermitteln. Eine darüber hinaus gehende Übermittlung anderer Daten an andere Stellen ist nicht über $\mathbb{} 299$ SGB V zu legitimieren. Vielmehr bedarf es in diesen Fällen einer gesonderten Rechtsgrundlage.

Erfolgt eine Übermittlung von Sozialdaten i.R.v. $\mathbb{S} 299$ Abs. 1a SGB V, sind die versichertenbezogenen Daten grundsätzlich zu pseudonymisieren ( 299 Abs. 1a S. 3 i.V.m. Abs. 1 S. 4 Nr. 1 SGB V). Dies erfolgt entweder bei den Leistungserbringern selbst ( $\$ 299$ Abs. 2 S. 3 SGB V), bei einer vom G-BA beauftragten eigenständigen Stelle ( $\$ 299$ Abs. 2S. 3 SGB V) oder nach einer Vollerhebung über die ebenfalls vom G-BA beauftragte Vertrauensstelle ( $\$ 11$ Teil 1 Quesü-RL). Nur in den Richtlinien und Beschlüssen des G-BA geregelten Ausnahmefällen ist eine Pseudonymisierung entbehrlich. Darüber hinaus sieht das Gesetz grundsätzlich nur eine stichprobenweise Datenerhebung vor, sodass eine Vollerhebung ebenfalls nur im Rahmen der in den Richtlinien und Beschlüssen des G-BA geregelten Ausnahmefällen möglich ist ( $\$ 299$ Abs. 1a S. 3 i.V.m. Abs. 1 S. 4 Nr. 1 SGB V).

\subsection{Nutzung im Rahmen der Datentransparenz (\$ $303 a$ ff. SGB V)}

Die $\mathbb{s}$ 303a ff. SGB V schaffen eine Ermächtigungsgrundlage zur Übermittlung der im Rahmen des Risikostrukturausgleichs vom Bundesversicherungsamt erhobenen Daten in pseudonymisierter Form an das DIMDI in seiner Funktion als Datenaufbereitungs- und Vertrauensstelle. Mit Ausnahme des $\$ 303 \mathrm{~b}$ Abs. 2 SGB V, der die Ermächtigungsgrundlage für die Übermittlung verschlüsselter Regionalkennzeichen durch die Krankenkassen an das Bundesversicherungsamt enthält, schaffen die $\$ \mathbb{S} 303 \mathrm{a}$ ff. SGB V ausschließlich eine Rechtsgrundlage für die Übermittlung von Daten des Risikostrukturausgleichs durch das Bundesversicherungsamt, nicht jedoch für Übermittlung von Abrechnungsdaten durch die Krankenkassen. Folglich bilden auch die $\$ \$ \$ ~ 303 a \mathrm{ff}$. SGB V keine geeignete Ermächtigungsgrundlage.

\subsubsection{4 Übermittlung zu Zwecken der Forschung und Planung (S 75 SGB X)}

Gemäß $\mathbb{7} 75$ Abs. 1 SGB X ist die Übermittlung von Sozialdaten zulässig, sofern sie für ein bestimmtes Vorhaben der wissenschaftlichen Forschung im

135 In der Fassung vom 20.04.2017. 
Sozialleistungsbereich, der wissenschaftlichen Arbeitsmarkt- und Berufsforschung oder der Planung im Sozialleistungsbereich durch eine öffentliche Stelle im Rahmen ihrer Aufgaben erforderlich ist, schutzwürdige Interessen des Betroffenen nicht beeinträchtigt oder vom öffentlichen Interesse an der Forschung/Planung erheblich überwogen werden und die zuständige oberste Bundes- oder Landesbehörde die Übermittlung zuvor genehmigt hat ( $\$ 75$ Abs. 4 SGB X).

Die bisher vergleichsweise streng gefassten Anforderungen an ein im Vorhinein bestimmtes Forschungsvorhaben hat der Gesetzgeber unter Berücksichtigung der „Bedeutung der Forschung für die gesamtgesellschaftliche Entwicklung " ${ }^{136}$ teilweise gelockert. Mit den neuen Regelungen verfolgt der Gesetzgeber das Ziel, sozialdatenbasierter Forschung weitere rechtliche Möglichkeiten zu eröffnen, die die Effektivität und Langfristigkeit der Forschungsergebnisse fördern, wobei ein ,angemessener Ausgleich zwischen den Erfordernissen der Forschung auf der einen Seite und dem berechtigten Interesse der einzelnen betroffenen Personen an dem Schutz ihrer persönlichen Daten auf der anderen Seite“ hergestellt werden soll. ${ }^{137}$

Der hierzu eingefügte $\mathbb{5} 75$ Abs. 4a SGB X lautet:

„Ergänzend zur Übermittlung von Sozialdaten zu einem bestimmten Forschungsvorhaben nach Absatz 1 Satz 1 kann die Verwendung dieser Sozialdaten auch für noch nicht bestimmte, aber inhaltlich zusammenhängende Forschungsvorhaben des gleichen Forschungsbereiches beantragt werden. Die Genehmigung ist unter den Voraussetzungen des Absatzes 4 zu erteilen, wenn sich der Datenempfänger gegenüber der genehmigenden Stelle verpflichtet, auch bei künftigen Forschungsvorhaben im Forschungsbereich die Genehmigungsvoraussetzungen einzuhalten. Die nach Absatz 4 Satz 1 zuständige Behörde kann vom Antragsteller die Vorlage einer unabhängigen Begutachtung des Datenschutzkonzeptes verlangen. Der Antragsteller ist verpflichtet, der nach Absatz 4 Satz 1 zuständigen Behörde jedes innerhalb des genehmigten Forschungsbereiches vorgesehene Forschungsvorhaben vor dessen Beginn anzuzeigen und dabei die Erfüllung der Genehmigungsvoraussetzungen darzulegen. Mit dem Forschungsvorhaben darf acht Wochen nach Eingang der Anzeige bei der Genehmigungsbehörde begonnen werden, sofern nicht die Cenehmigungsbehörde vor Ablauf der Frist mitteilt, dass für das angezeigte Vorhaben ein gesondertes Genehmigungsverfahren erforderlich ist.“

Somit soll der große Aufwand der Beantragung von Folgeforschungsvorhaben reduziert werden, wobei die in Erwägungsgrund 33 der DSGVO „getroffene Wertung, dass oftmals der Zweck der Verarbeitung personenbezogener Daten für Zwecke der wissenschaftlichen Forschung zum Zeitpunkt der Erhebung

136 BR-Drs. 430/18, S. 180.

137 BR-Drs. 430/18, S. 180. 
der personenbezogenen Daten nicht vollständig angegeben werden kann“, umgesetzt werden soll. ${ }^{13^{8}}$ Die Privilegierung ist dabei auf inhaltlich zusammenhängende Forschungsvorhaben des gleichen Forschungsbereichs begrenzt, wobei „einzelne Elemente der Forschungsfrage unterschiedlich“ sein können. ${ }^{139}$ Gleichzeitig soll durch eine hinreichende Bestimmtheit, und weitere Kontrollrechte eine Absenkung des Schutzniveaus verhindert werden. ${ }^{140}$ Zur weiteren Vereinfachung enthält Satz 5 eine Genehmigungsfiktion, sofern nicht vor Ablauf einer Frist von acht Wochen nach Eingang der vollständigen Unterlagen die Genehmigungsbehörde ein gesondertes Genehmigungsverfahren für erforderlich erklärt. Insbesondere letztere Regelung kann zu besserer Planungssicherheit und einer Beschleunigung der Verfahren führen. Gleichzeitig besteht hierbei das Risiko, dass ohne entsprechende Prüfung Sozialdaten verarbeitet werden und somit das Ziel der Verhinderung der Absenkung des Schutzniveaus konterkariert werden könnte. Der zurückhaltend formulierte Wortlaut spricht jedoch lediglich davon, dass mit dem Forschungsvorhaben „bereits begonnen“ werden darf. Ein nachträgliches behördliches Einschreiten bliebe möglich; vor dem Hintergrund der achtwöchigen Frist erscheint dieses Risiko jedoch angemessen.

Im Einzelnen stellt sich die Anwendung des $₫ 75$ SGB X wie folgt dar:

\section{Bestimmtes Vorhaben}

S 75 Abs. 1 S. 1 SGB X kann eine Datenübermittlung grundsätzlich nur legitimieren, wenn diese für ein bestimmtes Vorhaben erfolgt. Die Beschränkung auf ein bestimmtes Vorhaben schließt die Übermittlung für generelle, nicht näher bestimmte Forschungs- und Planungszwecke zunächst aus. ${ }^{141}$ Darüber hinaus erweitern $\mathbb{7 5}$ Abs. 2 und 4a SGB X ${ }^{142}$ die zulässige Übermittlung im Einzelfall. Sofern eine entsprechende Genehmigung vorliegt, können zum einen weitere Sozialdaten für die Bearbeitung von mit dem Ausgangsvorhaben inhaltlich zusammenhängenden Forschungsfolgefragen bzw. die ursprünglich übermittelten Sozialdaten auch für inhaltlich mit dem Ausgangsvorhaben in Zusammenhang stehende Forschungsvorhaben des gleichen Forschungsbereichs übermittelt werden.

138 BT-Drs. $18 / 12611$, S. 110.

139 BT-Drs. 18/12611, S. 110.

140 BT-Drs. 18/12611, S. 111.

141 Zur wortgleichen Fassung des $\$ 75$ Abs. 1 S. 1 SGB X a.F. Diering/Seidel, in: Diering/Timme, SGB X, 4. Aufl. 2016, 75 Rn. 4; BT-Drs. 12/5187, S. 40.

142 \ 75 Abs. 2 SGB X lautet: „Ergibt sich aus dem Vorhaben nach Absatz 1 Satz 1 eine Forschungsfrage, die in einem inhaltlichen Zusammenhang mit diesem steht, können hierzu auf Antrag die Frist nach Absatz 4 Satz 5 Nummer 4 zur Verarbeitung der erforderlichen Sozialdaten verlängert oder eine neue Frist festgelegt und weitere erforderliche Sozialdaten übermittelt werden." 


\section{Vorhaben im Bereich der wissenschaftlichen Forschung im Sozialleistungsbereich, der wissenschaftlichen Arbeitsmarkt- und Berufsforschung oder der Planung im Sozialleistungsbereich}

$\mathbb{\$} 75$ Abs. 1 S. 1 SGB X kann zudem nur als Ermächtigungsgrundlage herangezogen werden, wenn das Vorhaben im Bereich der wissenschaftlichen Forschung im Sozialleistungsbereich, der wissenschaftlichen Arbeitsmarkt-, sowie Berufsforschung (Nr. 1) oder der Planung im Sozialleistungsbereich durch eine öffentliche Stelle im Rahmen ihrer Aufgaben (Nr. 2) angesiedelt ist.

\section{Bereich der wissenschaftlichen Forschung im Sozialleistungsbereich}

Für die Subsumtion eines Vorhabens unter den Bereich der wissenschaftlichen Forschung im Sozialleistungsbereich ist es nicht erforderlich, dass sich das Forschungsvorhaben auf das Gebiet des Leistungsrechts beschränkt oder konzentriert. ${ }^{143}$ Vielmehr können auch Forschungsvorhaben mit volkswirtschaftlichem, soziologischem oder medizinischem Schwerpunkt darunter fallen, sofern etwaige Erkenntnisse mit gewisser Wahrscheinlichkeit auch im Sozialleistungsbereich von Bedeutung sein können. ${ }^{144}$ Demzufolge kann über $\$ 75$ Abs. 1 SGB X die Übermittlung von personenbezogenen Sozialdaten für eine Vielzahl von (bestimmten) Forschungsvorhaben legitimiert werden, sofern die zu erwartenden Erkenntnisse für den Sozialleistungsbereich von Bedeutung sein können. Dieser zu erwartende Nutzen muss im Genehmigungsantrag ( $\$ 75$ Abs. 4 SCB X) im Einzelfall konkret dargelegt werden. Je weiter der eigentliche Forschungszweck dabei vom Sozialleistungsbereich entfernt ist, desto umfassender sind die Anforderungen an die Darlegung dieses Nutzens für den Sozialleistungsbereich. ${ }^{145}$

\section{\75 Abs. 1 Nr. 2 SGB X}

Des Weiteren können Sozialdaten gemäß $\mathbb{7 5}$ Abs. 1 Nr. 2 SGB X auch zum Zweck der Planung im Sozialleistungsbereich übermittelt werden. Der Begriff der Planung im Sozialleistungsbereich umfasst dabei im Wesentlichen die Bedarfs-, Bereitstellungs- und Finanzplanung. ${ }^{146}$

\section{Empfangende Stelle}

Für die in $\$ 75$ Abs. 1 S. 1 Nr. 1 SGB X genannten Zwecke der wissenschaftlichen Forschung im Sozialleistungsbereich kann die Übermittlung sowohl an eine

143 Krause, in: Schlegel/Voelzke, jurisPK-SGB X, 3. Aufl., 2013, § 75 Rn. 28.

144 Rombach, in: Hauck/Noftz, SGB X, Stand: 08/2017, $\$ 75$ Rn. 14; Krause, in: Schlegel/Voelzke, jurisPK-SGB X, 2013, \ 75 Rn. 28.

145 Rombach, in: Hauck/Noftz, SGB X, Stand: 08/2017, \75 Rn. 14

146 Krause, in: Schlegel/Voelzke, jurisPK-SGB X, 2013, \$75 Rn. 28; vgl. dazu weitergehend Kap. 1.4.3.2.3. 
öffentliche als auch an eine nicht-öffentliche Stelle erfolgen. Wesentliches Kriterium soll in diesem Zusammenhang allein die Seriosität der empfangenden Stelle und deren Unabhängigkeit, sprich Freiheit von rein kommerziellen Forschungsinteressen, sein. ${ }^{147}$ Die empfangende Stelle muss bei der Verarbeitung besonderer Kategorien personenbezogenen Daten - Gesundheitsdaten -

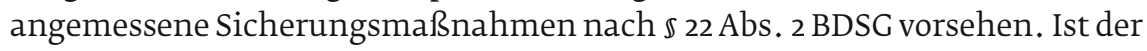
Empfänger der Daten eine nicht-öffentliche Stelle muss sich diese zudem gemäß $\mathbb{7} 75$ Abs. 4 S. 3 SGB X gegenüber der Genehmigungsbehörde vorab verpflichten, die Daten ausschließlich im Rahmen der vorgesehenen Zwecke zu verarbeiten. Zudem kann die genehmigende Behörde einer nicht-öffentlichen Stelle Auflagen erteilen, um die Einhaltung der gesetzlichen Vorgaben des $\mathbb{} 75$ SGB X sicherzustellen ( $\$ 75$ Abs. 5 SGB X). Aufsichtsbehörde für nicht-öffentliche Stellen ist gemäß $₫ 75$ Abs. 6 SGB X i.V.m. $\$ 40$ Abs. 1 BDSG die nach dem jeweiligen Landesrecht, der die nicht-öffentliche Stelle unterfällt, zuständige Behörde. Das sind regelmäßig die Landesbeauftragten für den Datenschutz.

Werden die Daten gemäß $\$ 75$ Abs. 1 S. 1 Nr. 2 SGB X zu Zwecken der Planung im Sozialleistungsbereich übermittelt, ist der Kreis der Empfänger auf öffentliche Stellen beschränkt, für die die Planung im entsprechenden Bereich im Rahmen ihrer Aufgaben liegt. Eine Übermittlung der Daten an eine nicht-öffentliche Stelle ist hier nicht zulässig.

\section{Zu übermittelnde Daten}

Gemäß des allgemeinen Erforderlichkeits- und Datenminimierungsprinzips (Art. 5 Abs. 1 lit. c] DSGVO) schreibt auch $\mathbb{} 75$ Abs. 1 S. 1 SGB X vor, dass eine Übermittlung von Sozialdaten nur zulässig ist, soweit sie für das jeweilige Vorhaben erforderlich ist. Demnach wird die Übermittlung auf die für das konkrete Forschungs- oder Planungsvorhaben erforderlichen Daten begrenzt. Gleiches gilt auch, sofern die Übermittlung weiterer Sozialdaten nach $\mathbb{} 75$ Abs. 2 SGB X genehmigt wurde.

\$ 67d Abs. 2 SGB X erweitert die Übermittlungsbefugnis auf für das konkrete Vorhaben nicht erforderliche Daten, wenn diese mit den Sozialdaten, für die eine Übermittlungsbefugnis besteht, derart verbunden sind, dass eine Trennung nicht oder nur mit unvertretbarem Aufwand verbunden ist und schutzwürdige Interessen des Betroffenen oder eines Dritten nicht überwiegen. Ein unvertretbarer Aufwand ist dabei nur zu bejahen, wenn die Daten derart physisch miteinander verbunden sind, dass die Trennung einen erheblichen Zeit-, Personal- und/oder Kostenaufwand bedeuten würde. ${ }^{148}$ Dies ist nicht der Fall, wenn die Daten noch getrennt vorliegen oder mit technischen Hilfsmitteln

147 Rombach, in: Hauck/Noftz, SGB X, Stand: 08/2017, \$ 75 Rn. 16.

148 Diering/Seidel, in: Diering/Timme, SGB X, 4. Aufl., 2106, §67d Rn. 4. 
ohne größere Mühe herauskopiert werden können. ${ }^{149}$ Hinsichtlich der Abwägung der mit den ggf. bestehenden schutzwürdigen Interessen der Betroffenen, bedarf es einer Einzelfallbetrachtung, bei der die Art der Daten und die Folgen für den Betroffenen von erheblicher Bedeutung sind. Gemäß $₫ 67 \mathrm{~d}$ Abs. 2 Hs. 2 SGB X dürfen die überschießenden Daten ausschließlich mit übermittelt, darüber hinaus aber nicht verändert oder genutzt werden. $\$ 67 \mathrm{~d} A \mathrm{bs} .2$ Hs. 2 SGB X statuiert insoweit ein absolutes Verwertungsverbot für die überschießenden Daten ${ }^{150}$.

\section{Keine Beeinträchtigung schutzwürdiger Interessen des Betroffenen oder überwiegendes öffentliches Interesse}

Die Übermittlung darf schutzwürdige Interessen der betroffenen Person nicht beeinträchtigen ( $\$ 75$ Abs. 1 S. 1 SGB X). Die Bestimmung schutzwürdiger Interessen erfolgt unter Berücksichtigung objektiver und subjektiver Gesichtspunkte mit Blick auf den Grad der Sensibilität der Daten und die möglichen Folgen einer Übermittlung. ${ }^{151}$ Beachtlich sind in diesem Zusammenhang nicht nur die Interessen des Einzelnen, sondern auch aller Betroffenen zusammen. ${ }^{152}$ Da vorliegend regelmäßig besondere Kategorien personenbezogener Daten betroffen sind, sind aufgrund der besonderen Schutzwürdigkeit der Daten jedoch keine allzu hohen Anforderungen an ein schutzwürdiges Interesse zu stellen. Liegt ein schutzwürdiges Interesse vor, ist eine Beeinträchtigung der Schutzwürdigkeit gegeben, sofern die Übermittlung aus objektiver Sicht zu Nachteilen für den Betroffenen führen kann oder der Betroffene der Übermittlung bereits widersprochen hat. ${ }^{153}$ Eine pauschale Aussage, wann eine Beeinträchtigung eines schutzwürdigen Interesses gegeben ist, ist folglich nicht möglich. Vielmehr bedarf es einer genauen Betrachtung des Einzelfalls.

Werden die schutzwürdigen Belange des Einzelnen beeinträchtigt, kann eine Übermittlung dennoch stattfinden, sofern das öffentliche Interesse an der Übermittlung und dem dahinterstehenden Vorhaben die Interessen des Betroffenen erheblich überwiegt. Dabei sind aufseiten des öffentlichen Interesses der Nutzen und die Bedeutung des Vorhabens maßgeblich zu berücksichtigen. Ein überwiegendes Interesse liegt etwa vor, wenn das Vorhaben für die weitere Aufgabenerfüllung der übermittelnden Stelle erforderlich ist und das Vorhaben ohne die Übermittlung unmöglich würde. ${ }^{54}$ Denkbar ist die Annahme eines überwiegenden öffentlichen Interesses jedoch auch, wenn über die Ergebnisse ein erheblicher Nutzen für die Allgemeinheit zu erwarten ist.

149 Rombach, in: Hauck/Noftz, SGB X, Stand: 08/2017, \67d Rn. 107.

150 Rombach, in: Hauck/Noftz, SGB X, Stand: 08/2017, § 67d Rn. 110.

151 Rombach, in: Hauck/Noftz, SGB X, Stand: 08/2017, \& 75 Rn. $27 f$.

152 Rombach, in: Hauck/Noftz, SGB X, Stand: 08/2017, \75 Rn. 25.

153 Krause, in: Schlegel/Voelzke, jurisPK-SGB X, 2013, § 75 Rn. 44.

154 Krause, in: Schlegel/Voelzke, jurisPK-SGB X, 2013, \$ 75 Rn. 47. 


\section{Anonymisierung}

$\mathbb{} 75$ Abs. 1 S. 1 SGB X legitimiert eine Übermittlung personenbezogener Sozialdaten nur, sofern dies für den jeweiligen Zweck erforderlich ist. Über die ausdrückliche Erwähnung des Erforderlichkeitsgebots hebt diese Vorschrift den Vorrang der Übermittlung anonymisierter Daten hervor. ${ }^{155}$ Als für den Betroffenen mildere Form der Verarbeitung hat die Anonymisierung der Sozialdaten auch für den Bereich der Forschungs- und Planungszwecke Priorität. ${ }^{156}$ Eine Verarbeitung personenbezogener Sozialdaten kann nur erforderlich sein, wenn eine Anonymisierung nicht in Betracht kommt. Für besondere Kategorien personenbezogener Daten, wie die im Rahmen der Abrechnungsdaten regelmäßig betroffenen Gesundheitsdaten, regelt $₫ 75 \mathrm{Abs}$. 3 S. 2 SGB X darüber hinaus, dass die Daten im Rahmen der Verarbeitung durch den Empfänger zu anonymisieren sind, sobald dies nach dem Forschungszweck möglich ist. Dies entspricht der Forderung des Art. 89 Abs. 1 Satz 4 DSGVO. Darüber hinaus statuiert $₫ 284$ Abs. 3 S. 2 SGB V eine Pflicht zur Löschung des Versichertenbezugs, wenn es sich bei den zu übermittelnden Daten um Daten handelt, die gemäß $\mathbb{} 295$ Abs. 1b S. 1 SGB V von den Leistungserbringern an die Krankenkassen übermittelt wurden. ${ }^{157}$

\section{Einwilligung}

\ 75 Abs. 1 S. 2 SGB X regelt die Priorität der Einwilligung, wonach auch im Bereich der in $₫ 75$ Abs. 1 S. 1 SGB X genannten Vorhaben eine Übermittlung grundsätzlich nur mit der vorherigen Einwilligung des Betroffenen zulässig ist, es sei denn, dies ist dem Verantwortlichen unzumutbar oder es handelt sich ausschließlich um explizit genannte Daten, die ausschließlich zu Befragungszwecken eingesetzt werden sollen ( $\$ 75$ Abs. 1 S. 3 SGB X). ${ }^{158}$ Dabei müssen auch hier die oben genannten, grundlegenden Anforderungen an die Einwilligung erfüllt sein, wobei ein besonderes Augenmerk auf die Gestaltung der Einwilligung im Hinblick auf die Freiwilligkeit zu richten ist. ${ }^{159}$ Im Hinblick auf die Zweckbestimmung ist das konkrete Vorhaben im Rahmen der Einwilligung zu benennen und soweit möglich zu beschreiben. Für Einwilligungen in die Übermittlung zu Forschungszwecken besteht gemäß $₫ 67 \mathrm{~b}$ Abs. 3 SGB X daneben jedoch auch die Möglichkeit eines Broad Consent. ${ }^{160}$

155 Vgl. Bieresborn, in: von Wulffen/Schütze, SGB X, 8. Aufl. 2014, § 75 Rn. 6.

156 Rombach, in: Hauck/Noftz, SGB X, Stand: 08/2017, \$ 75 Rn. 40; Bieresborn, in: von Wulffen/Schütze, SGB X, 8. Aufl. 2014, § 75 Rn. 6.

157 Vgl. hierzu oben Kap. 1.4.3.2.3, Abschnitt „Anforderungen an i.R.d. § 295 Abs. 1b S. 1 SGB V übermittelte Daten".

158 Vgl. oben Kap. 1.4.3.2.3, Abschnitt: „Vorrang der Einwilligung (\$ 75 Abs. 1 S. 2 SGB X)“.

$159 \mathrm{Vgl}$. oben Kap. 1.4.3.3.

160 Hierzu sogleich Kap. 3.2. 


\section{Entbehrlichkeit}

Gemäß $\$ 75$ Abs. 1 S. 3 SGB X ist die Einwilligung des Betroffenen immer dann entbehrlich, wenn lediglich Angaben zum Namen, Vornamen, Anschrift, Telefonnummer sowie zu für die Einleitung des Forschungsvorhabens zwingend erforderlichen Strukturmerkmalen der Person - hierzu gehören allgemeine Merkmale wie Alter, Herkunft, Berufsstatus ${ }^{161}$ - ausschließlich für Befragungen übermittelt werden. Mit dieser Ausnahmeregelung für Kontaktdaten zum Zweck der Befragung soll einer frühzeitigen, nicht mehr korrigierbaren Selektion und Verzerrung der Forschungsergebnisse begegnet werden . ${ }^{162}$ Sollen die Daten jedoch über die bloße Befragung hinaus verarbeitet werden, bedarf die Übermittlung einer gesonderten Ermächtigungsgrundlage.

Darüber hinaus ist die Einwilligung entbehrlich, sofern die Einholung für die übermittelnde Stelle unzumutbar ist. ${ }^{163}$ Die Darlegungslast für die Unzumutbarkeit der Einholung der Einwilligungen obliegt der übermittelnden Stelle. ${ }^{164}$ Diese muss im Rahmen des Genehmigungsverfahrens die Gründe für die Unzumutbarkeit im Einzelnen darlegen.

\section{Einwilligungserfordernis nach $\$ 76$ SGB X (Schweigepflichtverlängerung)}

Trotz einer möglichen Entbehrlichkeit der datenschutzrechtlichen Einwilligung nach $\mathbb{S} 75$ Abs. 1 SGB X, besteht ein Erfordernis nach einer Schweigepflichtentbindung (strafrechtlichen Einwilligungserklärung), sofern bei der Übermittlung solche Sozialdaten an einen Dritten übertragen werden, die die

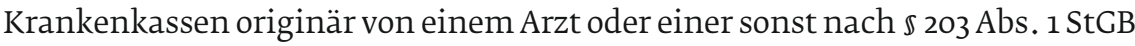
geheimnisverpflichteten Person erhalten haben. So heißt es in $\mathbb{5} 76 \mathrm{Abs} .1$ SGB X:

„Die Übermittlung von Sozialdaten, die einer in $\$ 35$ des Ersten Buches genannten Stelle von einem Arzt oder einer Ärztin oder einer anderen in $\$ 203$ Absatz 1 und 3 des Strafgesetzbuches genannten Person zugänglich gemacht worden sind, ist nur unter den Voraussetzungen zulässig, unter denen diese Person selbst übermittlungsbefugt wäre."

Für die von Ärzten übermittelten Abrechnungsdaten ist höchstrichterlich entschieden, dass diese Daten dem Schutzbereich des $\mathbb{} 203$ StGB unterfallen. ${ }^{165}$ Folglich darf der Sozialleistungsträger die Daten, die ihm beispielsweise von einem Arzt aufgrund der $\$ \$ 284 \mathrm{ff}$. SGB V übermittelt worden sind, nur an Dritte

161 Krause, in: Schlegel/Voelzke, jurisPK-SGB X, 2013, \$ 75 Rn. 50.1; Rombach, in: Hauck/Noftz, SGB X, Stand: 08/2017, \& 75 Rn. 40b.

162 Rombach, in: Hauck/Noftz, SGB X, Stand: 08/2017, § 75 Rn. 40 b.

163 Siehe dazu im Detail Kapitel 1.4.3.2.3, Abschnitt „Vorrang der Einwilligung“.

164 Rombach, in: Hauck/Noftz, SGB X, Stand: 08/2017, § 75 Rn. 33.

165 BGH, Urteil vom 10.07.1991, VIII ZR 296/90. 


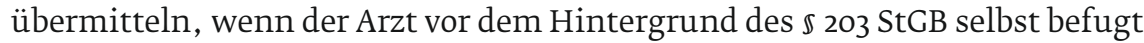
wäre, diese Daten zu den entsprechenden Zwecken an den Dritten zu offenbaren. Eine Befugnis zur Offenbarung i.S.d. $\mathbb{2} 203$ StGB kann etwa anzunehmen sein, wenn der Betroffene eine Schweigepflichtentbindung hinsichtlich der Weitergabe der Daten erteilt hat oder eine gesetzliche Offenbarungspflicht oder -befugnis besteht. Letzteres ist für Ärzte regelmäßig nicht gegeben. Für Ärzte selbst existieren keine gesetzlichen Ermächtigungsgrundlagen, die eine Weitergabe personenbezogener Daten zu Forschungszwecken erlauben. Im Einzelfall kann eine datenschutzrechtliche Norm zwar eine Befugnis zur strafrechtlichen Offenbarung enthalten, wenn sich dies aus dem Wortlaut der betreffenden Norm klar ergibt. Das Bundesverfassungsgericht sieht $\$ 203$ StCB wegen des Merkmals „unbefugt“ als sog. Blankettnorm an ${ }^{166}$, sodass auch landesrechtliche Gesetze und gegebenenfalls auf einer ausreichenden Ermächtigung beruhende Verordnungen eine Befugnis einräumen können. ${ }^{167}$ Selbst die in einigen Landeskrankenhausgesetzen enthaltenen Forschungsklauseln betreffen im Regelfall nur die krankenhausinterne Forschung und nicht die Weitergabe der Daten zu Forschungszwecken an externe Dritte. Diese Frage ist je nach einschlägigem Landesrecht im Einzelfall zu prüfen. Regelmäßig bedarf es einer Schweigepflichtentbindungserklärung des Betroffenen, um den Arzt von seiner Schweigepflicht zu entbinden und die Übermittlung zu legitimieren.

Zwar wird nicht verlangt, dass die Rechtfertigungsgründe von Erstübermittlung und Zweitübermittlung identisch sind, sodass der an $₫ 76$ SGB X gebundene Zweitübermittler auch andere Rechtfertigungsgründe heranziehen kann, auf die sich der originär an $\$ 203$ StGB gebundene Erstübermittler hätte stützen können. ${ }^{168}$ Ein Sozialleistungsträger wird aufgrund der verlängerten ärztlichen Schweigepflicht aber in der Regel einer Schweigepflichtentbindung des Betroffenen benötigen, um die personenbezogenen Abrechnungsdaten der Ärzte zu Forschungszwecken an einen Dritten übermitteln zu können. Die Ausnahmetatbestände des $₫ 75$ Abs. 1 S. 2 und 3 SGB X helfen hier nicht weiter. Vielmehr besteht dieses aus der strafrechtlich verankerten Geheimhaltungspflicht herrührende Zustimmungserfordernis neben dem datenschutzrechtlichen Einwilligungserfordernis ( $\$ 35 \mathrm{Abs}$. 2a SGB I). Ist die Einwilligung aus datenschutzrechtlicher Sicht entbehrlich, bedarf es bei Abrechnungsdaten dennoch einer Schweigepflichtentbindung. Die Schweigepflichtentbindung muss sich im Falle der Entbehrlichkeit der datenschutzrechtlichen Einwilligung jedoch nicht an den datenschutzrechtlichen Anforderungen messen lassen. Vielmehr kommt auf dieser Ebene auch eine formlose, gar konkludente Erklärung in Betracht. Da dem Sozialleistungsträger aber auch hier im Streitfall die Beweislast obliegt, ist eine schriftliche oder elektronische Einwilligung

167 Kircher, Der Schutz personenbezogener Gesundheitsdaten im Gesundheitswesen, 2016, S. 112 m.w.N.

168 Bieresborn, in: v. Wulffen/Schütze, 8. Aufl. 2014, SGB X § 76 Rn. 4-17a. 
empfehlenswert. Inhaltlich muss die Erklärung lediglich die Entbindung von der Schweigepflicht in Bezug auf die zu benennenden Daten/Geheimnisse umfassen.

Es sei an dieser Stelle nochmals darauf hingewiesen, ${ }^{169}$ dass eine Weitergabe von pseudonymisierten Daten hingegen in aller Regel ohne Schweigepflichtentbindung zulässig sein wird, da es insofern an einer Offenbarung im Sinne des $\mathbb{2 0 3}$ StCB fehlen wird. Eine solche liegt nämlich dann nicht vor, wenn für den Empfänger eines Geheimnisses die Kenntnis der betreffenden Person nicht möglich ist. Auch hier gilt also, dass eine Pseudonymisierung für den Empfänger eines Datensatzes mit einer Anonymisierung gleichzusetzen sein kann und eine persönlichkeitsrechtliche Relevanz daher nicht mehr fortbesteht.

\section{Maßnahmen nach $\$ 22$ BDSG}

Sofern wie bei der Übermittlung von Abrechnungsdaten besondere Kategorien personenbezogener Daten - Gesundheitsdaten - an Dritte übermittelt werden, fordert $₫ 67$ b Abs. 1 S. 4 SGB X, dass neben dem Erfordernis einer entsprechenden Ermächtigungsgrundlage die Gewährleistung der in $\$ 22$ Abs. 2 BDSG beschriebenen besonderen Schutzmaßnahmen durch den Dritten sichergestellt sind (siehe hierzu bereits Kap. 1.4.4).

\section{Behördliche Genehmigung}

Gemäß $\mathbb{7 5}$ Abs. 4 SGB X bedarf die Übermittlung von Sozialdaten zu den in Absatz 1 und 2 genannten Forschungs- und Planungszwecken für bestimmte Vorhaben der behördlichen Genehmigung. Zuständige Genehmigungsbehörde ist die jeweilige oberste Bundes- oder Landesbehörde, die für den Bereich, aus dem die Daten herrühren, zuständig ist, mithin das im Einzelfall zuständige Bundes- oder Landesministerium ( $\$ 75$ Abs. 4 S. 1 SGB X). Entsprechend der Ermächtigung in $₫ 75$ Abs. 4 S. 2 SGB X hat das Gesundheitsministerium diese Aufgabe für Anträge der GKV Anfang 2012 auf das Bundesversicherungsamt übertragen, ${ }^{170}$ sodass entsprechende Genehmigungen seither beim Bundesversicherungsamt zu beantragen sind.

\section{Genehmigungsantrag}

Der Genehmigungsantrag ist durch die empfangende Stelle zu stellen. ${ }^{171} \mathrm{Er}$ muss Ausführungen zu den wesentlichen Punkten des Vorhabens und den benötigten Daten enthalten. Dementsprechend ist zunächst das bestimmte Vorhaben und der betroffene Forschungs-/Planungsbereich i.S.d. $\mathbb{} 75$ Abs. 1 S. 1

169 Vergleiche bereits einleitend unter Kap. 1.4.2.

170 Rombach, in: Hauck/Noftz, SGB X, Stand: 08/2017, \& 75 Rn. 41 .

171 BT-Drs. 12/5187, S. 40. 
SGB X möglichst genau darzustellen. Soweit es sich um ein Forschungsvorhaben im Sozialleistungsbereich handelt, welches aufgrund des Forschungsgegenstands nicht eindeutig dem Sozialleistungsbereich zugeordnet werden kann, weil der Forschungsschwerpunkt in einem anderen Bereich liegt, ist zudem darzulegen, dass die zu erwartenden Ergebnisse auch für den Sozialleistungsbereich von Relevanz sein können. ${ }^{172}$ Darüber hinaus ist darzulegen, welche Sozialdaten benötigt werden, in welchen Phasen des Vorhabens diese relevant werden, welcher Personenkreis betroffen sein wird und für welchen Zeitraum die Daten zu dem entsprechenden Zweck verarbeitet werden sollen. ${ }^{173}$ Des Weiteren sind im Antrag Ausführungen dazu zu machen, dass die Betroffeneninteressen nicht beeinträchtigt werden oder inwiefern das öffentliche Interesse an dem Vorhaben diese überwiegt. Sofern die Übermittlung ohne Einwilligung und Anonymisierung erfolgen soll, sind die Cründe für die Unzumutbarkeit der Einholung der Einwilligungen sowie die einer Anonymisierung entgegenstehenden Gründe darzulegen. ${ }^{174}$

Wird der Antrag entsprechend $₫ 75$ Abs. 2 SGB X auf die Genehmigung der Übermittlung weiterer Sozialdaten für die Bearbeitung von Forschungsfolgefragen erstreckt, muss der inhaltliche Zusammenhang mit dem Ausgangsvorhaben dargelegt werden. Da der von $₫ 75$ Abs. 2 SGB X geforderte inhaltliche Zusammenhang dabei eher eng auszulegen ist, sind im Einzelfall detaillierte Angaben notwendig. Die Gesetzesbegründung nennt in diesem Zusammenhang beispielhaft die Änderung oder Erweiterung einzelner Elemente des Forschungsvorhabens, die den Charakter des Vorhabens als solchen nicht verändern. ${ }^{175}$

Werden, wie dies bei Abrechnungsdaten der Fall ist, besondere Kategorien personenbezogener Daten übermittelt, muss der Datenempfänger Angaben zu dem von ihm vorgehaltenen angemessenen und spezifischen Maßnahmen zur Wahrung der Interessen der Betroffenen i.S.d. \$ 22 BDSG machen. Bei der Übermittlung der Daten an eine nicht-öffentliche Stelle hat der Empfänger zudem eine Verpflichtungserklärung dahingehend abzugeben, dass dieser die Daten entsprechend $\$ 78$ Abs. 1 S. 1 SGB X nur zu dem beantragten Zweck verarbeiten wird.

Zudem ist gemäß $₫ 75$ Abs. 1 S. 4 SGB X ein Datenschutzkonzept vorzulegen.

\section{Genehmigungsbescheid}

Liegt ein ordnungsgemäßer Antrag nach $₫ 75$ Abs. 4 SGB X vor und sind alle Voraussetzungen des $₫ 75$ Abs. 1 SGB X - sowie bei der erweiterten Genehmigung

172 Vgl. Kap. 1.5.3.2.4, Abschnitt: „Bereich der wissenschaftlichen Forschung im Sozialleistungsbereich“.

173 Rombach, in: Hauck/Noftz, SGB X, Stand: 08/2017, \$ 75 Rn. 45.

174 Rombach, in: Hauck/Noftz, SGB X, Stand: 08/2017, § 75 Rn. 45.

175 BT-Drs. 18/12611, S. 118. 
der Absätze 2 bzw. 4a - erfüllt, hat die Behörde die Genehmigung ohne einen Ermessensspielraum gegenüber dem Empfänger zu erteilen. ${ }^{176}$ Die Entscheidung ergeht in Form eines Verwaltungsakts und ist dem Leistungsträger ebenfalls bekanntzugeben. ${ }^{177}$ Gemäß $₫ 75$ Abs. 4 S. 5 SGB X muss die Genehmigung den Dritten, an den die Daten übermittelt werden, die Art der zu übermittelnden Sozialdaten und den betroffenen Personenkreis sowie die wissenschaftliche Forschung oder die Planung, zu der die übermittelten Sozialdaten verwendet werden dürfen genau bezeichnen. Darüber hinaus muss aus der Genehmigung der Tag hervorgehen, bis zu dem die übermittelten Sozialdaten verarbeitet werden dürfen. Zudem kann die Behörde unmittelbar mit dem Erlass des Bescheides oder auch nachträglich Auflagen erteilen, ergänzen oder ändern. Ist der Empfänger eine nicht-öffentliche Stelle, hat die Behörde die Einhaltung der Vorgaben des $\mathbb{5} 75$ SCB X über eben solche Auflagen sicherzustellen.

Neben dem Antrag ist der Genehmigungsbehörde zudem ein Datenschutzkonzept vorzulegen, aus dem hervorgeht, dass der Empfänger die technischen und organisatorischen Anforderungen des Datenschutzes i.S.d. Art. 32 DSGVO sowie den Grundsatz der Datenminimierung erfüllt. ${ }^{178}$

\section{Löschung}

Grundsätzlich sind personenbezogene Daten zu löschen, sobald sie für den jeweiligen Zweck nicht mehr erforderlich sind ( $\$ 304$ Abs. 3 SGB V i.V.m. $\mathbb{8} 84$ Abs. 2 SGB X a.F.; vgl. Art. 17 Abs. 1 lit. a) DSGVO) ${ }^{179}$. Demzufolge müssten die Daten nach Abschluss des Forschungsvorhabens bzw. nach Ablauf des im Genehmigungsbescheid genannten Zeitpunkts unmittelbar gelöscht werden. Um eine Nachprüfung der Forschungsergebnisse auf der Grundlage der aufbereiteten Daten sowie die Verarbeitung der Daten für weitere Forschungsvorhaben gemäß Abs. 2 zu ermöglichen, bestimmt $₫ 75$ Abs. 4 S. 6 SGB X jedoch, dass die Daten nach Ablauf des von der Genehmigungsbehörde für die Verarbeitung bestimmten Vorhabens bis zu zehn Jahre aufbewahrt werden können. Dabei obliegt es der Genehmigungsbehörde durch Auflagen zu bestimmen, wie lang und bei welcher Stelle (übermittelnde oder empfangende Stelle) die Daten gespeichert werden können. ${ }^{180}$ Zudem kann sie weitere Auflagen für die verlängerte Speicherung erteilen.

176 Rombach, in: Hauck/Noftz, SGB X, Stand: 08/2017, \$ 75 Rn. 47.

177 Rombach, in: Hauck/Noftz, SGB X, Stand: 08/2017, \& 75 Rn. 47.

178 BT-Drs. 18/12611, S. 118.

$179 \S 304$ SGB V ist anpassungsbedürftig, da infolge der unmittelbaren Geltung des Art. 17 DS-GV0 auch $\S 84$ SGB X angepasst wurde und der Löschbefehl nicht mehr in $\$ 84$ SGB X normiert ist, sodass die Verweisung fehlerhaft ist. An dieser Stelle sei darauf hingewiesen, dass der Entwurf zum 2. DSAnpUG-EU die Streichung des Verweises auf $\$ 84$ Abs. 2 SGB X entsprechend vorsieht, vgl. BT-Drs. 19/4674, S. 143.

180 BT-Drs. 18/12611, S. 119. 


\section{Möglichkeiten der Verweigerung der Herausgabe der Daten an eine externe Einrichtung trotz Vorliegens einer Einwilligung}

\$ 75 SGB X erklärt die Datenübermittlung bei Vorliegen aller genannten Voraussetzungen für zulässig. Damit ist der Leistungsträger im Einzelfall befugt, die Daten zu übermitteln. Eine Verpflichtung lässt sich aus dem Wortlaut des $\mathbb{7} 75$ SGB X nicht herleiten. ${ }^{81}$ Auch die Einwilligung des Betroffenen verpflichtet den Verantwortlichen nicht, die Daten tatsächlich zu den genannten Zwecken zu übertragen. Vielmehr stellt die Einwilligung lediglich eine Befugnis dar.

Fraglich ist jedoch, ob sich aus der behördlichen Genehmigung zumindest mittelbar eine Pflicht zur vollumfänglichen Übertragung der in der Genehmigung benannten Daten ergibt. Zwar stellt auch die Genehmigung zunächst lediglich eine Übermittlungsbefugnis und keine -verpflichtung dar. Jedoch kann sich die Genehmigung entsprechend dem Sinn und Zweck des gegenständlichen Vorhabens zu einer Verpflichtung verdichten. So kann die Verweigerung der Herausgabe einzelner Daten im Rahmen eines genehmigten Vorhabens die Gefahr einer selektiven Informations-/Forschungs-/Planungsgrundlage bergen und so zu einer Verzerrung der Forschungsergebnisse führen. Dies entspräche dann jedoch nicht mehr dem behördlich genehmigten Vorhaben. Sofern die Genehmigung folglich bestimmte Datensätze bestimmter Personenkreise für ein Vorhaben umfasst, sind diese auch in Gänze zu übermitteln. Nur sofern eine solche Gefahr der Selektivität und der Ergebnisverzerrung im Einzelfall plausibel ausgeschlossen werden kann, ließe sich entsprechend der obigen Ausführungen gegen eine Übermittlungspflicht argumentieren. Darüber hinaus kann sich eine Übermittlungspflicht auch aus den konkreten vertraglichen Regelungen zwischen der übermittelnden und der empfangenden Stelle ergeben.

\section{Zwischenergebnis}

Zu Zwecken der wissenschaftlichen Forschung und Planung im Sozialleistungsbereich kommt eine Übermittlung von Sozialdaten gemäß $₫ 75$ SGB X in Betracht, soweit die Genehmigung der zuständigen Genehmigungsbehörde vorliegt ( $\$ 75$ Abs. 4 SGB X). Diese Genehmigung ist zu erteilen, wenn die Voraussetzungen des $₫ 75 \mathrm{Abs}$. 1 SCB X, sowie bei der erweiterten Genehmigung der Absätze 2 und 4a vorliegen. Dabei muss der Schwerpunkt des Vorhabens im Bereich der Forschung nicht zwingend auf dem Sozialleistungsbereich liegen. Ausreichend ist, dass die Forschungsergebnisse dem Sozialleistungsbereich mit gewisser Wahrscheinlichkeit dienlich sind.

Gemäß $\mathbb{7} 75$ Abs. 1, $\$ 76$ SGB X ist für die Übermittlung von Sozialdaten in diesen Bereichen grundsätzlich die Einwilligung der Betroffenen erforderlich.

181 Hase, DuD 2011, 875 (876). 
Zwar sieht $\$ 75$ Abs. 1 SGB X auf datenschutzrechtlicher Ebene eine Ausnahme von diesem Erfordernis vor, sofern die Einholung unzumutbar ist. Aufgrund der Ausdehnung der Voraussetzungen der Geheimhaltungspflicht nach $\mathbb{2 0 3}$ StCB in $\mathbb{7} 76$ SGB X bedarf es jedoch - entsprechend den Anforderungen, die etwa ein Arzt einhalten müsste - auch in diesen Fällen grundsätzlich einer Einwilligung in Form einer Schweigepflichtentbindung.

Entsprechend dem Grundsatz der Datenminimierung dürfen Sozialdaten nur in einem Umfang übermittelt werden, der für die Zweckerreichung im jeweiligen Vorhaben auch erforderlich ist. Eine unbegründete, wahllose Datenübermittlung dürfte daher regelmäßig im Genehmigungsverfahren scheitern. Zudem sind die Daten auch hier zu anonymisieren, soweit es der Forschungszweck zulässt. Darüber hinaus darf die Übermittlung keine schutzwürdigen Interessen der Betroffenen beeinträchtigen, es sei denn, das öffentliche Interesse an dem Vorhaben überwiegt diese erheblich.

Der Empfänger muss über technische und organisatorische Maßnahmen sicherstellen, dass die Rechte und Interessen der Betroffenen hinreichend geschützt werden und hat diese Maßnahmen im Genehmigungsverfahren über ein Datenschutzkonzept und entsprechende Nachweise zu belegen. Dabei ist es für den Bereich der wissenschaftlichen Forschung unerheblich, ob die empfangende Stelle eine öffentliche oder eine nicht-öffentliche Stelle ist. Letztere haben im Rahmen des Genehmigungsverfahrens lediglich zusätzliche Verpflichtungserklärungen zur zweckgebundenen Verarbeitung abzugeben. Für den Bereich der Planung kommen als Empfänger hingegen nur öffentliche Stellen in Betracht, da $\$ 75$ Abs. 1 S. 1 Nr. 2 SGB X fordert, dass die Planung zum Aufgabenbereich der jeweiligen Stelle gehört.

In jedem Fall unterliegt auch die externe Stelle mit Erhalt der Sozialdaten dem Sozialgeheimnis nach $\mathbb{} 35$ SCB I und hat dafür Sorge zu tragen, alle mit den Daten befassten Mitarbeiter entsprechend zu verpflichten.

\subsubsection{Einwilligung}

Die Übermittlung der Daten an eine externe Einrichtung zu Zwecken der wissenschaftlichen Forschung, der Planung im Sozialleistungsbereich oder der Qualitätssicherung kann alternativ auf Grundlage einer Einwilligung des Betroffenen erfolgen. Dabei sind die oben genannten allgemeinen und speziellen/sozialrechtlichen Anforderungen an die Einwilligung zu beachten ${ }^{182}$. Wie bereits oben unter A.III.3.a) (s. Kap. 1.4.3.3.1) ausgeführt, ist aufgrund des bestehenden Abhängigkeitsverhältnisses zwischen Leistungsträger und $\mathrm{Be}-$ troffenem besonderes Augenmerk auf die Gestaltung der Einwilligung im Hinblick auf die Freiwilligkeit der Erklärung zu richten.

182 Vgl. oben Kap. 1.4.3.3. 


\subsubsection{Auftragsverarbeitung}

Neben der Übermittlung der Daten an eine eigenständige externe Stelle ist es zudem denkbar, dass sich der Leistungsträger eines Auftragsverarbeiters bedient, um die personenbezogenen Abrechnungs-/Sozialdaten zu Zwecken der Forschung, Planung oder Qualitätssicherung zu verarbeiten. Eine externe Stelle ist immer dann als Auftragsverarbeiter einzuordnen, wenn sie die Daten im Interesse und auf Weisungen des Verantwortlichen verarbeitet und keine wesentlichen Entscheidungen über Zweck und Mittel der Datenverarbeitung trifft. ${ }^{183}$

Art. 28 DSGVO enthält spezifische Regeln zur Ausgestaltung und Zulässigkeit der Auftragsverarbeitung. Darüber hinaus enthält $\$ 80$ SGB X speziell für den Bereich des Sozialdatenschutzes weitere Vorgaben zur Auftragsverarbeitung. Da Art. 28 DSGVO keine Öffnungsklauseln in Bezug auf die Auftragsverarbeitung enthält, ist fraglich, inwiefern diese bereichsspezifische nationale Norm mit der DSGVO in Einklang steht. Da die Auftragsverarbeitung jedoch als (ausgelagerter) Verarbeitungsvorgang des Verantwortlichen zu verstehen ist, können weitergehende Regelungen durch die Mitgliedsstaaten getroffen werden, sofern innerhalb der DSGVO (Art. 6-10 DSGVO) Öffnungsklauseln für den konkreten Bereich der Datenverarbeitung existieren. ${ }^{184}$ Die Regelung des $\mathbb{s} 80$ SGB X ist folglich über die Öffnungsklauseln der Art. 6. Abs. 1 lit. e), Abs. 2, Abs. 3 S, 3, Art. 9 Abs. 2 lit. b), h), j) DSGVO europarechtskonform.

\subsection{Ermächtigungsgrundlage}

Fraglich ist zunächst, ob die Weitergabe der Sozialdaten an einen Auftragsverarbeiter ebenso wie die Weitergabe der Daten an einen Dritten einer gesonderten gesetzlichen Ermächtigungsgrundlage bedarf.

\section{Datenschutzrechtliche Ermächtigung}

Nach dem BDSG a.F. kam der Auftragsverarbeitung eine Privilegierung in der Form zu, dass eine Weitergabe der Daten an den Auftragsverarbeiter nicht als Übermittlung, sondern vielmehr als interne Nutzung der Daten verstanden wurde, die einer gesonderten Ermächtigungsgrundlage nicht bedurfte. Eine Weitergabe an den Auftragsverarbeiter war immer dann zulässig, wenn die Verarbeitung durch den Verantwortlichen selbst rechtmäßig war. Begründet wurde dies mit den Definitionen der $\mathbb{s} 3$ Abs. 4 Nr. 3, 3 Abs. 8 S. 2 und 3 BDSG a.F., nach denen eine Übermittlung nur bei der Weitergabe der Daten an einen Dritten vorliegt, der Auftragsverarbeiter aber gerade kein Dritter ist.

183 Hartung, in: Kühling/Buchner, DS-GVO, 2017, Art. 4 Nr. 8 Rn. 7.

184 Hartung, in: Kühling/Buchner, DS-GVO, 2017, Art. 28 Rn. 113. 
Die DSGVO greift diese Definition der Übermittlung nicht auf, sondern erfasst diese als Unterfall der Verarbeitung in Form der Offenlegung (Art. 4 Nr. 2 DSGVO). Dabei liegt eine Offenlegung nach der DSGVO in jeder Weitergabe der Daten unabhängig von der Einordnung der empfangenden Stelle als Dritter oder Auftragsverarbeiter. ${ }^{185}$ Dennoch wird in der Literatur das Fortbestehen der Privilegierung der Auftragsverarbeitung mit Verweis auf die historische Entwicklung und systematische Stellung der Auftragsverarbeitung sowie der Bedeutung der Auftragsverarbeitung auch im Rahmen der DSGVO befürwortet. ${ }^{186}$ Insbesondere die Tatsachen, dass die DSGVO die Regelungen zur Auftragsverarbeitung im Wesentlichen aus der Datenschutz-Richtlinie, in deren Rahmen eine Privilegierung der Auftragsverarbeitung angelegt war ${ }^{187}$, übernommen hat und dass der Auftragsverarbeiter streng an die Weisungen des Verantwortlichen gebunden ist und Art. 28 DSGVO detaillierte Anforderungen an die Auftragsverarbeitung stellt, sprechen dafür, dass die Weitergabe von Daten auch nach der DSGVO keines gesonderten Erlaubnistatbestands bedarf. ${ }^{188}$ Ohne eine Privilegierung der Auftragsverarbeitung würden die zahlreichen besonderen Regelungen zur Auftragsverarbeitung in der DSGVO keinen Sinn ergeben. Folglich ist die Weitergabe der Abrechnungsdaten zu Zwecken der wissenschaftlichen Forschung, Planung im Sozialleistungsbereich sowie der Qualitätssicherung an einen Auftragsverarbeiter aus datenschutzrechtlicher Sicht zulässig, sofern der Verantwortliche selbst zu einer Datenverarbeitung zu den entsprechenden Zwecken legitimiert ist. ${ }^{189}$ Einer gesonderten Ermächtigungsgrundlage oder Einwilligung für die Weitergabe der Daten an den Auftragsverarbeiter bedarf es aus datenschutzrechtlicher Sicht daher nicht. ${ }^{190}$

\section{Strafrechtliche Befugnis}

Fraglich ist jedoch, inwiefern die Weitergabebefugnis aufgrund von $₫ 76$ SGB X durch den Geheimnisschutz nach $₫ 203$ StGB begrenzt wird. $\$ 76$ SGB X bestimmt, dass die Übermittlung von Sozialdaten, die der Leistungsträger von einer in $\mathbb{2} 203$ Abs. 1 und 3 StGB genannten, zur Geheimhaltung verpflichteten Stelle erhalten hat, nur zulässig ist, wenn die nach $₫ 203$ Abs. 1 und 3 StGB verpflichtete Person selbst zur Übermittlung befugt wäre. Verlangt wird dabei

185 Herbst, in: Kühling/Buchner, DS-GV0, 2017, Art. 4 Nr. 2 Rn. $30 f$.

186 Hartung, in: Kühling/Buchner, DS-GV0, 2017, Art. 28 Rn. 13ff.; Schmitz/von Dall'Armi, ZD 2016, 427 (429, 432); Schmidt/Freund, ZD 2017, 14; ebenso BayLDA, Auftragsverarbeitung nach der DS-GVO, Kurz-Papier vom 26.10.2016, 1. Nach anderer Ansicht ist die Zulässigkeit der Verarbeitung auch beim Auftragsverarbeiter zu prüfen, vgl. etwa Dovas, ZD 2016, 512 (516); eine solche dürfte in der Regel jedoch gerechtfertigt sein.

187 Schmidt/Freund, ZD 2017, 14 (15).

188 Hartung, in: Kühling/Buchner, DS-GVO, 2017, Art. 28 Rn. 13ff.; Schmidt/Freund, ZD 2017, 14 (15f.).

189 Zur rechtmäßigen Verarbeitung von Sozialdaten durch den Leistungserbringer vgl. Ausführungen zu Szenario 1 , s. Kap. 1.4.3.1.1.

190 Ebenso Unabhängigen Datenschutzbehörden des Bundes und der Länder (Datenschutzkonferenz - DSK), Kurzpapier Nr. 13, Auftragsverarbeitung, Art. 28 DS-GVO, Stand 16.02.2018, S. 2. 
nicht die Identität der Rechtfertigungsgründe von Erstübermittlung und Zweitübermittlung; der an $₫ 76$ SCB X gebundene Zweitübermittler kann also auch andere Rechtfertigungsgründe heranziehen, auf die sich der originär an $\sqrt{ } 203$ StCB gebundene Erstübermittler hätte stützen können. ${ }^{191}$

Ob davon aber nur die Weitergabe der Daten an einen Dritten oder auch die Weitergabe an einen Auftragsverarbeiter erfasst ist, ist bisher nicht geklärt.

\section{Jegliche Offenbarung i.S.d. \$ 203 StGB erfasst}

Der Begriff der Übermittlung stellt nach der DSGVO nicht mehr auf die Weitergabe der Daten an einen Dritten ab. Vielmehr ist nunmehr jegliche Übermittlung an einen Empfänger eine Form der Verarbeitung im Sinne der Offenlegung. Dementsprechend ließe sich auch die Weitergabe der Daten an einen Auftragsverarbeiter unter den Begriff der Datenübermittlung subsumieren. Zudem besagt $₫ 35 \mathrm{Abs}$. 2a SGB I, dass die Berufsgeheimnisse sowie die gesetzlichen Geheimhaltungspflichten unberührt bleiben und folglich umfassend neben den datenschutzrechtlichen Regelungen fortgelten sollen. Daraus ließe sich folgern, dass die gesetzliche Geheimhaltungspflicht des $\$ 203$ StCB auch bei einer Verlängerung umfassend neben den datenschutzrechtlichen Pflichten gelten soll. Ist dies der Fall, wäre dem Leistungsträger jedoch jegliche Offenbarung gegenüber einem nicht gemäß $\$ 203$ StGB zum Mitwissen Befugten und somit auch gegenüber dem Auftragsverarbeiter ohne die Einwilligung/ Schweigepflichtentbindungserklärung des Betroffenen unzulässig.

\section{Nur die Weitergabe an Dritte erfasst}

$\$ 76$ SGB X beschränkt nach seinem Wortlaut - wie bereits bisher $\$ 76 \mathrm{Abs} .1$ SGB X a.F. - lediglich die Befugnis zur Übermittlung von besonders schutzwürdigen Sozialdaten. Entsprechend der Definition des Übermittlungsbegriffs in $\mathbb{3}$ Abs. 4 Nr. 3 BDSG a.F., nach der eine Übermittlung nur an Dritte, nicht aber an Auftragsverarbeiter erfolgen kann, wäre die Verlängerung des $\mathbb{} 203$ StGB nicht auf die Weitergabe der Daten an den Auftragsverarbeiter zu übertragen. Für diese Auffassung spricht, dass mit dem 2. SGBÄndG der Wortlaut der Norm von „Offenbarung“, die jegliche Preisgabe von Daten an andere erfasst, auf Übermittlung umgestellt wurde und damit vermeintlich bewusst ein engerer Kreis gezogen wurde. ${ }^{192}$ Dass der Gesetzgeber im Rahmen des $\mathbb{} 76$ SGB X keine Ausführungen zu einem veränderten Geltungsbereich des $\$ 76$ SGB X gemacht hat, spricht dafür, dass ihm die Problematik nicht bewusst und keine Änderung gewollt war. Zudem ist zwar die Eigenschaft des Empfängers, Dritter zu sein, für eine Übermittlung nach der DSGVO nicht mehr

191 Bieresborn, in: v. Wulffen/Schütze, 8. Aufl. 2014, SGB X \ 76 Rn. 4-17a.

192 So im Ergebnis bei einem Vergleich zu § 39 BDSG a.F. zur aktuellen Fassung Rombach, in: Hauck/Noftz, SGB X, Stand: 08/2017, \ 76 Rn. 9. 
maßgeblich. Jedoch ist dies für eine Weitergabe der Daten an den Auftragsverarbeiter auch nicht relevant, da dieser Vorgang lediglich einen Teil der internen Verarbeitung durch den Verantwortlichen darstellt, der zulässig ist, wenn die Verarbeitung durch den Verantwortlichen ihrerseits zulässig ist und die gesetzlichen Vorgaben der Auftragsverarbeitung eingehalten werden. Die Weitergabe der Daten an einen Auftragsverarbeiter wäre folglich auch mit Blick auf $\mathbb{2 0 3}$ StCB ohne Einwilligung des Betroffenen zulässig.

\section{Exkurs: Übermittlungsbefugnis des Geheimnisträgers nach $§ 203$ StGB}

Ginge man von der erstgenannten Ansicht (s. Kap. 1.5.3.4.1 Abschnitt „Jegliche Offenbarung i.S.d. $\$ 203$ StGB erfasst") aus, wäre auch die Weitergabe der Daten durch den Leistungserbringer an den Auftragsverarbeiter nur zulässig, wenn der nach $\$ 203$ StGB unmittelbar Geheimnisverpflichtete - beispielsweise der Arzt - selbst zur Weitergabe berechtigt wäre. Dies war nach $\$ 203$ StGB a.F. nur der Fall, wenn der Arzt sich auf eine entsprechende gesetzliche Ermächtigungsgrundlage, die Einwilligung des Patienten oder einen sonstigen strafrechtlichen Rechtfertigungsgrund stützen könnte. Dies ist bei der Übermittlung zu Forschungs- und Planungszwecken jedoch regelmäßig nicht der Fall. ${ }^{193}$

Diese Problematik löst sich auch nach Inkrafttreten des nunmehr geltenden $\mathbb{} 203$ StGB nicht vollständig. Zwar wird durch $\mathbb{2} 203$ Abs. 3 StCB der Kreis der zum Mitwissen Befugten auf sonstige Personen, die an der beruflichen oder dienstlichen Tätigkeit des Geheimnisverpflichteten mitwirken, also etwa bestimmte Auftragsverarbeiter, erweitert. Diese Erweiterung erstreckt sich jedoch nur auf Auftragsverarbeiter, die an der beruflichen oder dienstlichen Tätigkeit des Geheimnisverpflichteten mitwirken. Stellt man hier auf den Arzt und die für ihn geltenden Rechtsgrundlagen ab, erweist sich die Übertragung des Geheimnisschutzes nach $\mathbb{} 203$ StGB als problematisch. Denn für einen Vertragsarzt gehört die Forschung oder Planung im Sozialleistungsbereich wohl nicht zu seiner beruflichen Tätigkeit, sodass ein Mitwirken nach dem nunmehr geltenden $₫ 203$ Abs. 3 StCB abzulehnen ist und eine zulässige Offenbarung ausscheidet. Für den Arzt einer Uniklinik gehört die Forschung hingegen zumeist zum Bereich seiner dienstlichen Tätigkeit, sodass ein Mitwirken an dieser i.S.d. $\$ 203$ StCB möglich und eine Weitergabe der Daten an einen Auftragsverarbeiter zulässig ist. Eine solche Differenzierung dürfte in der Praxis kaum zu handhaben sein. Auch dies spricht letztendlich gegen eine Übertragung der Geheimnisverpflichtung bei der Weitergabe der Daten an einen Auftragsverarbeiter.

193 Hierzu siehe oben Kap. 1.5.3.2.4, Abschnitt „Einwilligungserfordernis nach § 76 SGB X (Schweigepflichtverlängerung)“. 


\section{Zwischenergebnis}

Wie die Prüfüberlegung (s. Kap. 1.5.3.4.1, Abschnitt „Exkurs: Übermittlungsbefugnis des Geheimnisträgers nach $\$ 203$ StCB“) zeigt, würde die Ausweitung des Geltungsbereichs des $\mathbb{5} 76$ SGB X auf Auftragsverarbeiter im Einzelfall zu erheblichen Subsumtionsproblemen führen, sodass auch dies dafür spricht, die Weitergabe der Daten an den Auftragsverarbeiter vom Anwendungsbereich des $\$ 76$ SGB X auszunehmen.

\subsection{Anforderungen nach $\S 80$ SGB $X$}

Bedient sich der Leistungsträger eines Auftragsverarbeiters, sind die zusätzlichen Anforderungen des $\mathbb{S} 80$ SGB X sowie des Art. 28 DSGVO zu beachten.

Gemäß $\mathbb{8} 8$ Abs. 1 SGB X hat der Verantwortliche die Einschaltung eines Auftragsverarbeiters der für ihn zuständigen Fach- oder Rechtsaufsicht (Bundesversicherungsamt oder Sozialministerien der Länder) schriftlich oder elektronisch anzuzeigen. Die Anzeige muss dabei Angaben

- zum Auftragsverarbeiter,

- zu dessen technischen und organisatorischen Maßnahmen sowie

- zu den erteilten ergänzenden Weisungen,

- der Art der betroffenen Daten und Personenkreise

- der Aufgabe, deren Erfüllung die Einschaltung dient sowie

- zum Abschluss etwaiger Unterauftragsverhältnisse

enthalten. Sofern der Auftragsverarbeiter seinerseits eine öffentliche Stelle ist, hat dieser seine Rechts- oder Fachaufsicht gleichermaßen rechtzeitig von der Beauftragung zu unterrichten. Die Rechtzeitigkeit der Anzeige soll der Aufsichtsbehörde die Möglichkeit der Stellungnahme oder Beratung vor Abschluss des Auftrages ermöglichen und ist entsprechend der Umstände im Einzelfall zu berechnen. In jedem Fall hat sie vor Auftragserteilung zu erfolgen.

Eine Auftragserteilung an eine nicht-öffentliche Stelle ist gemäß $\mathbb{s} 80 \mathrm{Abs} .3$ SGB X nur zulässig, wenn bei dem Verantwortlichen selbst Störungen im Betriebsablauf zu befürchten wären oder die übertragenen Arbeiten beim Auftragsverarbeiter erheblich kostengünstiger besorgt werden können. ${ }^{194}$ Insbesondere im Bereich der Forschung sind durchaus Vorhaben denkbar, bei denen der Aufwand Störungen im Betriebsablauf der Leistungsträger begründen oder die bei nicht-öffentlichen Stellen mit entsprechender Ausrüstung/Ausstattung

194 Nach dem Entwurf für das 2. DSAnpUG-EU soll dies nicht gelten, wenn Dienstleister in der Informationstechnik, deren absolute Mehrheit der Anteile oder deren absolute Mehrheit der Stimmen dem Bund oder den Ländern zusteht, mit vorheriger Genehmigung der obersten Dienstbehörde des Verantwortlichen beauftragt werden; BT-Drs. 19/4674, S. 154. Die Regelung soll insbesondere der Förderung der Umsetzung der vom Bundeskabinett beschlossenen Vorhaben zur IT-Konsolidierung in der Bundesverwaltung dienen; BT-Drs. 19/4674, S. 402. 
kostengünstiger durchgeführt werden können. Dabei ist im Rahmen der Anzeige an die Behörde der Fach- oder Rechtsaufsicht eine nachvollziehbare Darlegung im Einzelfall erforderlich. Allgemein ist es aufgrund der erheblichen Restriktionen durchaus von Vorteil, öffentliche Stellen als Auftragsverarbeiter einzubinden. Ausgenommen von dieser Beschränkung sind lediglich Auftragsverhältnisse über die Prüfung und Wartung automatisierter Verfahren oder von Datenverarbeitungsanlagen, bei denen ein Zugriff auf Sozialdaten nicht gänzlich ausgeschlossen werden kann (\$ 8o Abs. 5 SGB X).

Abschließend unterwirft $\mathbb{8} 80$ Abs. 4 SGB X den Auftragsverarbeiter der Aufsicht und den Befugnissen des Bundes- oder Landesdatenschutzbeauftragten für den Datenschutz und die Informationsfreiheit bzw. der nach Landesrecht zuständigen Aufsichtsbehörde sowie den Straf- und Bußgeldvorschriften der Is 85 und 85 a SGB $X$.

Darüber hinaus regelt $₫ 80$ Abs. 2 SGB X, dass eine Auftragserteilung nur zulässig ist, wenn die Datenverarbeitung im Inland, in einem Mitgliedsstaat der EU oder des EWR, der Schweiz oder einem Staat, der die Kommission ein angemessenes Schutzniveau per Beschluss nach Art. 45 DSGVO bescheinigt hat, verarbeitet werden. Mit dieser Beschränkung soll sichergestellt werden, dass sensible Sozialdaten nicht in einen unsicheren Drittstaat gelangen und dort einem geringeren Schutz und weitergehenden Verarbeitungsmöglichkeiten unterliegen. ${ }^{195}$

\subsection{Anforderungen nach Art. 28 DSGVO}

Die DSGVO enthält in Art. 28 umfangreiche Vorgaben für die Umsetzung einer wirksamen Auftragsverarbeitung.

Zunächst muss die Auftragsverarbeitung auf einen zwischen den Parteien geschlossenen schriftlichen oder elektronischen Vertrag basieren. Diese Verträge können individuell gestaltet sein oder auf (noch zu erstellenden) Standardvertragsklauseln der Kommission oder der Aufsichtsbehörden beruhen. Notwendige Inhalte des Vertrages sind:

- Gegenstand und Dauer der Verarbeitung,

- Art und Zweck der Verarbeitung,

- die Art der personenbezogenen Daten,

- die Kategorien betroffener Personen sowie

- die Pflichten und Rechte des Verantwortlichen und

- die Pflichten des Auftragsverarbeiters,

wobei Letztere in Art. 28 Abs. 3 S. 2 DSGVO näher bestimmt sind (z.B. Weisungsgebundenheit, Verschwiegenheitsverpflichtung der Mitarbeiter, Unterstützung des Verantwortlichen). Die Beschreibung der Verarbeitung (Gegenstand, 
Art, Zweck, betroffene Daten und Personen) sind „angemessen ausführlich“ $\mathrm{zu}$ beschreiben. ${ }^{196} \mathrm{Es}$ ist darauf $\mathrm{zu}$ achten, dass die Zweckbestimmung eindeutig beschrieben und erkennbar dem Verantwortlichen zugeordnet werden kann. ${ }^{197}$ Aus der Beschreibung heraus muss ein Dritter wie etwa eine Datenschutzbehörde die Verarbeitungstätigkeit hinreichend klar einsehen können. Bei Pflichten und Rechten des Verantwortlichen ist an das Recht, über Löschung und Berichtigung der Daten und Auskünfte an Betroffene zu entscheiden, die Prüfung der generellen Zulässigkeit und Rechtmäßigkeit einer Datenverarbeitung wahrzunehmen und ausreichend Weisungen zu erteilen, zu denken. ${ }^{198}$ Darüber hinaus steht es den Parteien frei, weitere Regelungen zu treffen. ${ }^{999}$

Daneben ist eine grundlegende Voraussetzung, dass die ausgewählten Auftragsverarbeiter hinreichend Garantien dafür bieten, dass geeignete technische und organisatorische Maßnahmen vorgehalten und eingesetzt werden, sodass die Verarbeitung im Einklang mit den Anforderungen der Verordnung erfolgt und der Schutz der Rechte der betroffenen Person gewährleistet ist. Dabei erfordert der Begriff der „Garantie“ zumindest ausreichende (vertragliche) Verpflichtungen sowie Nachweise des Auftragsverarbeiters, die eine sorgfältige und gewissenhafte Erbringung der Tätigkeit unter Einhaltung der Vorgaben der DSGVO wahrscheinlich machen. ${ }^{200}$ Künftig kann der Nachweis hinreichender Garantien zudem unter Einhaltung genehmigter Verhaltensregeln gem. Art. 40 DSGVO oder eines genehmigten Zertifizierungsverfahrens gem. Art. 42 DSGVO nachgewiesen werden. Der Verantwortliche bleibt dabei auch nach Erteilung des Auftrags zur Begleitung und Kontrolle der Auftragsverarbeitung verpflichtet. ${ }^{201}$ Eine Zusammenarbeit mit Auftragsverarbeitern ist ab dem Zeitpunkt nicht mehr gestattet, ab dem entsprechende Garantien nicht mehr gewährleistet sind. ${ }^{202}$

Des Weiteren enthält Art. 28 DSGVO die ausdrückliche Verpflichtung des Auftragsverarbeiters, Daten nur auf dokumentierte Weisung des Verantwortlichen oder einer gesetzlichen Pflicht zu verarbeiten (Art. 28 Abs. 3 S. 2 lit. a] DSGVO) und Unterauftragsverhältnisse nur mit Einwilligung des Verantwortlichen und bei Abschluss von Art. 28 Abs. 3 DSGVO entsprechenden Verträgen zu erteilen.

196 Hartung, in: Kühling/Buchner, DS-GV0, 2017, Art. 28 Rn. 65 unter Verweis auf Art.-29-Datenschutzgruppe Stellungnahme 1/2010 zu den Begriffen „für die Verarbeitung Verantwortlicher" und „Auftragsverarbeiter“, WP 169, 32.

197 Hartung, in: Kühling/Buchner, DS-GVO, 2017, Art. 28 Rn. 65.

198 Hartung, in: Kühling/Buchner, DS-GVO, 2017, Art. 28 Rn. 66.

199 Vgl. hierzu Hartung, in: Kühling/Buchner, DS-GV0, 2017, Art. 28 Rn. 80.

200 Hartung, in: Kühling/Buchner, DS-GV0, 2017, Art. 28 Rn. 56.

201 Hartung, in: Kühling/Buchner, DS-GV0, 2017, Art. 28 Rn. 60.

202 Martini, in: Paal/Pauly, Datenschutz-Grundverordnung, 2017, Art. 28 Rn. 21. 


\subsection{Zwischenergebnis}

Die Weitergabe von Sozialdaten an einen Auftragsverarbeiter im Rahmen der Durchführung eines bestimmten Vorhabens im Bereich der wissenschaftlichen Forschung oder der Planung im Sozialleistungsbereich ist ohne eine gesonderte Ermächtigungsgrundlage zulässig, sofern der Verantwortliche selbst zur Verarbeitung im entsprechenden Umfang befugt ist und die Voraussetzungen des $₫ 80$ SGB X sowie des Art. 28 DSGVO erfüllt sind. Dementsprechend ist ein Vertrag über die Auftragsverarbeitung mit den dort genannten Mindestangaben zu erstellen und die Beauftragung der jeweiligen Aufsichtsbehörde anzuzeigen. Dabei ergibt sich hinsichtlich der Auswahl des Auftragsverarbeiters eine Einschränkung dahingehend, dass nicht-öffentliche Stellen nur unter engen Voraussetzungen (bei dem Verantwortlichen sind ohne die Beauftragung Störungen im Betriebsablauf zu befürchten oder die Beauftragung ermöglicht eine erheblich kostengünstigere Bearbeitung) beauftragt werden können. Eine Ausnahme von dieser Restriktion besteht nach $₫ 80$ Abs. 5 SGB X lediglich für Auftragsverhältnisse zur Prüfung und Wartung automatisierter Verfahren der Datenverarbeitungsanlagen, auch wenn in diesem Rahmen ein Zugriff auf personenbezogene Daten nicht ausgeschlossen werden kann.

\subsubsection{Ergebnis}

Die Weitergabe von Sozialdaten an eigenverantwortlich handelnde externe Einrichtungen ist in den Bereichen der wissenschaftlichen Forschung, der Planung im Sozialleistungsbereich sowie der Qualitätssicherung in unterschiedlichem Umfang möglich.

Für die Qualitätssicherung ergibt sich die Befugnis zur Übermittlung allein aus der spezialgesetzlichen Norm des $\mathbb{2} 299$ SGB V, die eine Übermittlung ausschließlich nach den Vorgaben der Beschlüsse und Richtlinien des G-BA legitimiert.

Für den Bereich der wissenschaftlichen Forschung ist die Übermittlung an externe Stellen vornehmlich auf Grundlage von $\$ \mathbb{s} 75,76$ SGB X möglich. Dabei stellen die $\$ \$ 575,76$ SGB X jedoch detaillierte Regelungen auf, deren Einhaltung im Rahmen eines obligatorischen Genehmigungsverfahrens überprüft wird. So stellen die vorzuhaltenden technischen und organisatorischen Maßnahmen, der Erforderlichkeitsgrundsatz und das grundsätzliche Einwilligungserfordernis, welches aufgrund der Ausweitung des strafrechtlichen Geheimnisschutzes ( $\$ 203$ StCB) auf die Übermittlung der von Ärzten stammenden Abrechnungsdaten nach $\mathbb{7} 76$ SGB X - auch nicht unter den in $₫ 75$ SGB X genannten Voraussetzungen - entbehrlich ist, die wesentlichen Anforderungen an die Zulässigkeit des Vorhabens dar. Festzuhalten bleibt abschließend, dass die Übermittlung von Sozialdaten zum Zweck der wissenschaftlichen Forschung einem strengen Genehmigungsvorbehalt unterliegt und in jedem Fall der Einwilligung der Betroffenen (in Form einer Schweigepflichtentbindung) bedarf. 
Für die Planung im Sozialleistungsbereich gestaltet sich die Übermittlung an eigenverantwortlich handelnde, externe Stellen ebenso wie im Bereich der Forschung. Einschränkend kommt hier hinzu, dass die externe Stelle nur eine öffentliche Stelle sein kann, bei der die Planung zum zugewiesenen Aufgabenbereich gehört.

Soll die externe Stelle als Auftragsverarbeiter eingebunden werden, bedarf dies nach hier vertretener Auffassung grundsätzlich keiner gesonderten Ermächtigungsgrundlage. Vielmehr ist die Einbindung als Teil der Verarbeitungstätigkeit des Verantwortlichen zulässig, wenn dieser zu der geplanten Form der Verarbeitung legitimiert ist und die zusätzlichen Anforderungen des Art. 28 DSGVO sowie des $\$ 8$ o SGB X eingehalten werden. Neben dem Erfordernis eines Auftragsverarbeitungsvertrages ergibt sich aus $\mathbb{5} 80$ Abs. 3 SGB X eine Einschränkung für die Beauftragung von nicht-öffentlichen Stellen, die nur unter den beschriebenen, sehr engen Voraussetzungen einbezogen werden können.

\subsubsection{Zulässigkeit der Datenumgänge durch die externe Einrichtung (hinsichtlich der jeweiligen Zwecke)}

\subsubsection{Prüfungsmaßstab/Identifikation des einschlägigen Datenschutzregimes}

Agiert die externe Stelle als Verantwortlicher i.S.d. Art. 4 Nr. 7 DSGVO, stellt sich die Frage nach dem einschlägigen Datenschutzregime erneut.

\subsection{Rechtsnatur}

Allgemein richtet sich die Anwendbarkeit - abgesehen vom umfassenden Geltungsanspruch der DSGVO - im deutschen Datenschutzrecht nach der Rechtsnatur des Verantwortlichen. Für öffentliche Stellen des Bundes sowie für nicht-öffentliche Stellen gilt im Grundsatz das BDSG gemäß $\mathbb{1}$ Abs. 1 S. 1 Nr. 1 und S. 2 BDSG. Auf öffentliche Stellen der Länder ist im Grundsatz das jeweilige Landesdatenschutzgesetz anwendbar ${ }^{203}$, nur in den Fällen des $\mathbb{S} 1 \mathrm{Abs}$. 1 S. 1 Nr. 2 BDSG findet auch das BDSG auf diese Anwendung.

\subsection{Sonderfall Sozialdatenschutzrecht}

Gemäß $₫ 1$ Abs. 2 S. 1 BDSG gehen andere Rechtsvorschriften des Bundes über den Datenschutz dem BDSG vor. Während dies in den meisten Fällen lediglich punktuell regelnde Normen des bereichsspezifischen Datenschutzrechts betrifft, bei dem das BDSG außerhalb einer Tatbestandskongruenz als Auffangnorm weiter Anwendung findet, ist das Sozialdatenschutzrecht nach $\mathbb{3} 35$ SGB I i.V.m. $\$ \$ S 67$ ff. SGB X als Vollregelung konzipiert und regelt daher - mit

203 Auf eine Anpassung an die DS-GVO kommt es nicht an. Auch ohne Anpassung kommen die mit der DS-GV0 vereinbaren landesdatenschutzrechtlichen Vorschriften zur Anwendung. 
Ausnahme der DSGVO - die Verarbeitung von Sozialdaten abschließend ( $\$ 35$ Abs. 2 S. 1 SGB X). Ein Rückgriff auf das BDSG ist ausgeschlossen (vgl. $\$ 1$ Abs. 2 S. 2 BDSG).

Gleichzeitig knüpft das Sozialdatenschutzrecht nicht an der Rechtsnatur der verantwortlichen Stelle an, sondern nennt zunächst - wie auch nach bisherigem Recht - in $\mathbb{S} 35$ Abs. 1 SGB I die sozialdatenschutzverpflichteten Stellen. Während die Aufzählung im bisherigen $\$ 35$ SCB I a.F. abschließend und nicht analogiefähig ist, ${ }^{204}$ enthält nunmehr $\$ 35$ Abs. 6 SGB I eine Ausweitung der Sozialdatenschutzverpflichteten. Demnach sollen die Absätze 1 bis 5 des $\mathbb{3} 35$ SGB I „neben den in Absatz 1 genannten Stellen“ auch Anwendung auf solche Verantwortliche oder deren Auftragsverarbeiter finden, die Sozialdaten im Inland (nicht im Rahmen einer Niederlassung in einem anderen EU- oder EWR-Staat) oder im Rahmen der Tätigkeiten einer inländischen Niederlassung verarbeiten.

Diese Regelung ist problematisch. Die Anwendbarkeit eines bestimmten Datenschutzregimes wird nämlich nunmehr nicht mehr von der Anknüpfung an der Rechtsnatur oder einer festgelegten Anzahl an Stellen aus definiert, sondern knüpft am Charakter des Datums als Sozialdatum an. Der Begriff der Sozialdaten wird, wie oben bereits dargelegt, in $\$ 67$ Abs. 2 S. 1 SGB X definiert und nimmt hierbei insbesondere Bezug auf eine Verarbeitung durch eine ,in $\mathbb{} 35$ des Ersten Buches genannten Stelle“. Damit ergibt sich ein teilweiser Zirkelschluss, der das Potenzial einer ausufernden Anwendbarkeit des Sozialdatenschutzrechts, das unbestimmbar zu werden droht, birgt. Nach hier vertretener Ansicht ist eine restriktive Auslegung erforderlich, deren Bestimmung angesichts einer unpassenden Äußerung in den Gesetzgebungsmaterialien erschwert wird. Dort heißt es:

„Stellen, die nicht in Absatz 1 genannt und denen Sozialdaten übermittelt worden sind, sind gemäß $\$ 8$ Absatz 1 Satz 2 SGB X verpflichtet, die Daten in demselben Umfang geheim zu halten, wie eine in Absatz 1 genannte Stelle." 205

Diese Begründung setzt also - anders als der Normtext - voraus, dass zunächst eine Übermittlung durch eine Stelle nach $\mathbb{S} 35$ Abs. 1 SGB I vorliegt, die den Anknüpfungspunkt für das initiale Vorliegen von Sozialdaten liefert. Im Übrigen ist die Begründung allerdings ihrerseits widersinnig. Zum einen käme \$ 78 SGB X-eo ipso - ohnehin zur Anwendung und bedürfte keiner Ausweitung des Anwendungsbereichs des Sozialgeheimnisses insgesamt. Zum anderen beinhaltet der Wortlaut des $\mathbb{7} 78$ Abs. 1 S. 3 SGB X bei strenger Lesart auch nur eine Geheimhaltungspflicht, die im Sozialgeheimnis (s.o.) gerade nicht zu sehen ist. Im Sinne einer, auch von $\mathbb{} 35$ Abs. 2a SGB I ausdrücklich anerkannten, Unterscheidung von Geheimhaltungspflicht und Datenschutzrecht, ist

204 Steinbach, in: Hauck/Noftz/Becker, SGB I, Stand 07/2014, § 35 Rn. 22.

205 BT-Drs. 18/12611, S. 105; gemeint war hier aber wohl $\S 78$ Abs. 1 S. 2 SGB X a.F., der in der neuen Fassung $§ 78$ Abs. 1 S. 3 SGB X entspricht. 
es zunächst konsequent, wenn gefordert wird, dass ein Empfänger die Sozialdaten wie ein Sozialleistungsträger „hüten“ muss. So wird unter anderem gefordert, dass Empfänger, die keine originären Normadressaten des Sozialgeheimnisses sind, gem. $\mathbb{2}$ Abs. 2 Nr. 2 Verpflichtungsgesetz auf das Sozialgeheimnis zu verpflichten sind. ${ }^{206}$

Allerdings wurde bisher auch ein weitergehendes Verständnis vertreten, wonach der Datenempfänger im Sinne des $₫ 78$ Abs. 1 S. 2 SGB X a.F. in „dieselbe Schutzpflichtposition eingewiesen wird“ wie der "Datenabsender“ und dadurch zum ,abgeleiteten (derivativen) Normadressaten des $\$ 35$ SGB I“ werde. ${ }^{207}$ Das bedeute aber, dass diese Stelle die Daten auch wie ein Sozialleistungsträger unter den Voraussetzungen der $\mathbb{S} 568-76$ SGB X a.F. verwenden dürfte. ${ }^{208}$ Diese Beschränkung wird sowohl aus $₫ 78$ Abs. 1 S. 3 SGB X a.F. gefolgert, der ansonsten überflüssig wäre ${ }^{209}$, als auch aus $\mathbb{} 78$ Abs. 1 S. 1 SGB X, da ansonsten die dort verortete „absolute Zweckbindung“ umgangen werden könnte. ${ }^{210}$

Eben jene Argumentation könnte bei uneingeschränkter Anwendung des $₫ 35$ Abs. 6 SCB I aber beeinträchtigt werden, da dieser ausdrücklich auf die Absätze 1 bis 5 des $\mathbb{3} 35$ SGB I verweist und somit - vermittels $\mathbb{3} 35$ Abs. 2 SGB I - auch auf die $\$ \mathbb{S} 67 \mathrm{ff}$. SGB X.

Im Ergebnis ist daher eine dem Wortlaut prima facie zu entnehmende Ausdehnung des Anwendungsbereichs über $\mathbb{3} 3$ Abs. 6 SGB I abzulehnen.

Dennoch führt dies nicht dazu, dass hinsichtlich der Verarbeitungsvorgänge, die durch den Empfänger durchgeführt werden, wiederum auf das für ihn allgemein geltende Datenschutzrecht (z.B. das BDSC) zurückgegriffen werden müsste oder könnte. Vielmehr folgt auch die Verarbeitungsbefugnis zu dem konkreten Zweck, zu dem die Daten übermittelt wurden, unmittelbar aus $\$ 78$ Abs. 1 S. 1 SGB X („dürfen“). ${ }^{211}$ Die Geheimhaltungspflicht des $\mathbb{} 78$ Abs. 1 S. 3 SGB X umfasst im Übrigen auch die Einhaltung der weiteren Anforderungen an die Verarbeitung, wie etwa Anforderungen an technische und organisatorische Maßnahmen, entsprechend den Vorgaben des für Stellen nach $\mathbb{3} 35$ Abs. 1 SGB I geltenden Sozialdatenschutzes. ${ }^{212}$

\subsubsection{Gesetzliche Ermächtigung}

Somit gibt $₫ 78$ SGB X für die nach $\sqrt{ } 75$ SGB X übermittelten Daten vor, in welchem Umfang und auf welche Art und Weise die Verarbeitung durch den

\footnotetext{
206 Kunkel, in: Schlegel/Voelzke, jurisPK-SGB X, 2013, \ 78 SGB X, Rn. 20.

207 Kunkel, in: Schlegel/Voelzke, jurisPK-SGB X, 2013, \ 78 SGB X, Rn. 19.

208 Kunkel, in: Schlegel/Voelzke, jurisPK-SGB X, 2013, \& 78 SGB X, Rn. 19.

209 Kunkel, in: Schlegel/Voelzke, jurisPK-SGB X, 2013, § 78 SGB X, Rn. 19.

210 Rombach, in: Hauck/Noftz, SGB X, Stand: 08/2017, \ 78 Rn. 25.

211 Rombach, in: Hauck/Noftz, SGB X, Stand: 08/2017, \$ 78 Rn. $13 \mathrm{ff}$.

212 Rombach, in: Hauck/Noftz, SGB X, Stand: 08/2017, @ 78 Rn. 11.
} 
Empfänger der Daten erfolgen darf. Gemäß $\$ 78$ Abs. 1 S. 1 SGB X dürfen Empfänger, die nicht in $\mathbb{3} 35$ SGB I genannt sind, die Daten ausschließlich zu den Zwecken speichern, verändern, nutzen, übermitteln, in der Verarbeitung einschränken oder löschen, zu denen sie ihnen befugtermaßen übermittelt wurden (absolute Zweckbindung ${ }^{213}$ ). Dabei ist es unerheblich, ob die Stelle eine öffentliche oder nicht-öffentliche Stelle ist. Auch eine Weiterübermittlung durch die externe Einrichtung ist nur zulässig, wenn diese vom Zweck, der der Übermittlung durch den Sozialleistungsträger zugrunde liegt, umfasst sind. ${ }^{214}$

Sofern im Rahmen von $\$ 78$ SGB X a.F. noch darauf abgestellt wird, dass diese Befugnis nur für die konkrete Zweckbestimmung des einzelnen Vorhabens zu sehen ist und nicht auf die abstrakte Zweckbestimmung der Ermächtigungsgrundlage (hier $\$ 75$ SGB X) abgestellt werden darf ${ }^{215}$, darf dies im Rahmen der wissenschaftlichen Forschung nicht dahingehend missverstanden werden, dass die Verarbeitung durch den Empfänger nunmehr doch von einer konkreten Zweckbestimmung abhängt und der Broad Consent ausgeschlossen ist. Es darf lediglich nicht auf den allgemeinen Zweck der wissenschaftlichen Forschung (oder Planung) abgestellt werden, sondern auf die jeweilige Zweckbestimmung im Einzelnen. Ist der Broad Consent oder eine erweiterte Genehmigung nach $\mathbb{} 75$ Abs. 4a SGB X Rechtsgrundlage für die Übermittlung, muss auch die Verarbeitung durch den Empfänger in der jeweiligen Breite zulässig sein.

Neben der strengen Zweckbindung schreibt $\$ 78$ Abs. 1 S. 3 SGB X vor, dass der Empfänger der Daten die Daten in gleicher Weise wie der Verantwortliche nach $\mathbb{S} 35$ Abs. 1 SGB X geheim zu halten hat. Dies sichert zusätzlich zu der strengen Zweckbindung ab, dass die Daten in einem dem Sozialgeheimnis unterliegenden Bereich verbleiben und nicht unkontrolliert verbreitet werden.

Für nicht-öffentliche Stellen fordert $\$ 78$ Abs. 1 S. 2 SGB X, dass diese sich zuvor zu einer ebensolch zweckgebundenen Verarbeitung verpflichtet haben. ${ }^{216} \mathrm{Zu}$ dem muss die nicht-öffentliche Stelle sicherstellen, dass die dort beschäftigten Personen, die die Sozialdaten entsprechend verarbeiten, auf ihre Pflichten nach Absatz 1, sprich die Pflicht zur Geheimhaltung und die strenge Zweckbindung der Verarbeitung, hingewiesen wurden (\$78 Abs. 2 SGB X).

\subsubsection{Einwilligung}

Grundsätzlich kann die Einwilligung auf Grundlage von Art. 6 Abs. 1 lit. a) DSGVO und Art. 9 Abs. 2 lit. a) DSGVO - mangels Beschränkung der Einwilli-

213 Rombach, in: Hauck/Noftz, SGB X, Stand: 08/2017, \ 78 Rn. 17ff.

214 Rombach, in: Hauck/Noftz, SGB X, Stand: 08/2017, \$ 78 Rn. 21.

215 Rombach, in: Hauck/Noftz, SGB X, Stand: 08/2017, \& 78 Rn. 17.

216 An dieser Stelle sei darauf hingewiesen, dass diese Verpflichtung nach dem Entwurf zum 2. DSAnpUG-EU nur dann erforderlich sein soll, wenn die nicht-öffentliche Stelle um die Übermittlung ersucht hat, vgl. BTDrs. 19/4674, S. 154. 
gungsmöglichkeit durch den nationalen Gesetzgeber - auch im Sozialrecht nicht generell ausgeschlossen werden. Eine solche lässt sich auch nicht ohne Weiteres aus $₫ 78$ SGB X ablesen. Versteht man den $₫ 78$ SGB X jedoch in seiner strengen Zweckbindung eng, schließt dieser die Verwendung der übermittelten Sozialdaten zu Zwecken der Einholung einer Einwilligung aus, wenn dies nicht ausnahmsweise von der Ermächtigungsgrundlage, die der Übermittlung der Daten durch den Leistungsträger zugrunde lag - etwa bei der Einwilligung in Form des Broad Consent - erfasst ist. $\$ 78$ SGB X soll eine strenge Zweckbindung des Empfängers herbeiführen und ihn auf die Verarbeitung zu den sich aus der Befugnis der übermittelnden Stelle ergebenden Zwecken ermöglichen. Darüber hinaus soll dem Empfänger eine Verarbeitung nicht möglich sein. Dementsprechend sperrt $₫ 78$ SGB X für den Empfänger die Möglichkeit, eine Einwilligung für eine anderweitige Verarbeitung einzuholen. Dies ergibt sich daraus, dass der Empfänger die Daten nicht dazu verwenden darf, mit dem Betroffenen Kontakt aufzunehmen, um eine Einwilligung einzuholen. Dies ist vom Zweck, der die Übermittlung durch den Leistungsträger legitimierte, nicht mehr gedeckt.

\subsubsection{Auftragsverarbeitung}

Ist die externe Stelle selbst Auftragsverarbeiter, ist $₫ 78$ SGB X nicht relevant. Die Verarbeitungsbefugnisse richten sich in diesem Zusammenhang streng nach den Weisungen des Verantwortlichen und nach der für diesen bestehenden Verarbeitungsbefugnis. Eine darüberhinausgehende Verarbeitung durch den Auftragsverarbeiter ist weder für eigene noch für fremde Zwecke gestattet. Im Falle einer Unterbeauftragung durch eine als Auftragsverarbeiter agierenden externen Stelle perpetuiert sich die Verantwortlichkeit des ursprünglichen Auftraggebers.

Fraglich ist aber, ob die eigenverantwortliche, externe Stelle selbst befugt ist, einen Auftragsverarbeiter hinzuzuziehen und nach welchen Vorschriften sich die Auftragsverarbeitung dann richtet. Der Empfänger der Daten ist zu einer Verarbeitung entsprechend der Übermittlung der Daten an ihn zugrundeliegenden Zweckbestimmung berechtigt. Da die Weitergabe der Daten an einen Auftragsverarbeiter keinen eigenständigen Verarbeitungsvorgang darstellt, der einer gesonderten Ermächtigungsgrundlage bedarf, sondern vielmehr als Verarbeitung des Verantwortlichen im Rahmen seiner Befugnisse zu verstehen ist, kann eine Weitergabe der Daten an einen Auftragsverarbeiter erfolgen, sofern die Vorgaben des Art. 28 DSGVO sowie des $\$ 8$ o SGB X eingehalten werden. Letzterer ist vorliegend anwendbar, da der Auftraggeber nach $₫ 78$ SGB X verpflichtet ist und damit allen Beschränkungen, die das Sozialgeheimnis mit sich bringt, unterliegt. Dementsprechend richtet sich eine Auftragsverarbeitung, bei der die externe Stelle Auftraggeber ist, ebenfalls nach $₫ 80$ SGB X. 


\subsubsection{Anforderungen an „technische und organisatorische Maßnahmen“}

Gemäß $\$ 67 b$ Abs. 1 S. 4 SGB X gilt \$ 22 Abs. 2 BDSG entsprechend. Hierzu kann auf die vorangehenden Ausführungen verwiesen werden. ${ }^{217}$

\subsubsection{Genehmigungserfordernis und -anforderungen}

Im Rahmen von $\$ 75$ SGB X besteht ein Genehmigungserfordernis bereits für die Übermittlung an die externe Einrichtung. Die Verarbeitung durch diese bedarf darüber hinaus grundsätzlich keiner gesonderten Genehmigung. Eine Ausnahme kann im Fall der erweiterten Genehmigung nach $\$ 75$ Abs. 4a SGB X bestehen, bei der die Daten für einen bestimmten Bereich wissenschaftlicher Forschung genutzt werden sollen. Hier ist der Empfänger der Sozialdaten verpflichtet, die konkreten Vorhaben, zu denen die Daten verarbeitet werden sollen, vor dem Beginn des Vorhabens bei der Behörde, die die Genehmigung erteilt hat, anzuzeigen. Kommt diese Behörde zu dem Schluss, dass die Voraussetzungen nicht erfüllt sind, etwa weil der inhaltliche Zusammenhang fehlt, kann ein erneutes Genehmigungsverfahren nach $\mathbb{} 75$ Abs. 4 SGB X erforderlich werden.

Hinsichtlich der Auftragsverarbeitung erfordert $\sqrt{5} 80$ Abs. 1 SGB X vorab eine Anzeige gegenüber der zuständigen Rechts- oder Fachaufsichtsbehörde. ${ }^{218}$

\subsubsection{Ergebnis}

Aus $\mathbb{7} 78$ SGB X folgt sowohl die streng verstandene Zweckbindung hinsichtlich einer Verarbeitung der Sozialdaten durch den Empfänger, der wie eine in $\mathbb{S} 35$ SGB I genannte Stelle an das Sozialgeheimnis gebunden ist, als auch die Befugnis zur Verarbeitung zu diesem Zweck. Auf sonstige Erlaubnistatbestände - insbesondere solche des allgemeinen Datenschutzrechts - kann nicht zur Weiterverarbeitung zurückgegriffen werden. Alternativ wäre es zwar möglich, eine Einwilligung nach den Vorgaben der DSGVO einzuholen, allerdings kann die externe Stelle als durch $\$ 78$ Abs. 1 SGB X gebundener Empfänger die erhaltenen Daten nicht zum Zweck der Einholung einer solchen Einwilligung verwenden, sodass praktisch kaum ein Anwendungsfall bestehen dürfte.

Eine Auftragsverarbeitung richtet sich in jedem Fall nach den Vorgaben des $\mathbb{S} 8$ o SGB X, in dessen Rahmen sie auch zulässig ist.

Die Verarbeitung nach einer Übermittlung ist von der Genehmigung nach $\mathbb{} 75$ Abs. 4 SGB X umfasst. Im Falle der Auftragsverarbeitung ist eine Anzeige gegenüber der Rechts- oder Fachaufsicht erforderlich.

217 Hierzu siehe Kap. 1.4.4.

218 Siehe hierzu bereits Kap. 1.5.3.4.2; vgl. Rombach, in: Hauck/Noftz, SGB X, Stand: 08/2017, §80 SGB X Rn. 69. 


\subsection{Beurteilung von Szenario 3}

\subsubsection{Darstellung des Szenarios}

In diesem Szenario werden Sozialdaten von einer oder mehreren Einrichtungen der gesetzlichen Krankenversicherung zu übergeordneten Zwecken (z.B. medizinische Forschung oder übergeordnete Qualitätssicherungsaspekte) an eine externe Einrichtung (Datenplattform) übermittelt. Die externe Einrichtung wiederum stellt diese Sozialdaten für bestimmte Vorhaben Dritten zur Verfügung, entweder in Form einer weiteren Übermittlung oder indem anonyme Auswertungsergebnisse zu konkreten Fragestellungen herausgegeben werden.

Das Szenario 3 lässt sich somit in zwei Unterszenarios (3A und 3 B) unterteilen:

\subsubsection{Szenario $3 A$}

In der Variante $3 \mathrm{~A}$ erhält die externe Plattform Sozialdaten von unterschiedlichen Krankenkassen, führt diese zusammen und wertet sie selbst aus. Im Anschluss werden nur die anonymisierten Ergebnisse weitergegeben.

\subsubsection{Szenario $3 B$}

In Variante 3B erhält die externe Plattform Sozialdaten von den unterschiedlichen Krankenkassen, um diese nicht selbst auszuwerten, sondern mit den Daten der anderen Krankenkassen ggf. zusammenzuführen und zu Auswertungszwecken an eine abermals dritte Stelle zu übermitteln.

\subsubsection{Rechtfertigungsbedürftige Datenverarbeitungsvorgänge}

Wie auch in den vorangehenden Szenarien weisen wir daraufhin, dass lediglich die Übermittlung und weitere Verarbeitung personenbezogener Daten rechtfertigungsbedürftig ist. Liegen (faktisch) anonyme Daten, jedenfalls aus Sicht der empfangenden und weiterverarbeitenden externen Plattform sowie weiterer Empfänger vor, so sind diese ohne datenschutzrechtliche Restriktionen zulässig.

Vorliegend kommen im Übrigen bei jedem Unterszenario mehrere rechtfertigungsbedürftige Verarbeitungsschritte in Betracht.

\subsubsection{Szenario $3 A$}

Wie auch bei Szenario 2 ist hier jeweils eine Weitergabe der Daten durch die Krankenkasse an die Plattform erforderlich (s. Kap. 1.6.3.1). Anschließend werden die Daten zum Zweck der Auswertung durch die Plattform genutzt (s. Kap. 1.6.3.2). 


\subsubsection{Szenario $3 \mathrm{~B}$}

Wie auch in Szenario 3A müssen Sozialdaten von Krankenkassen an die externe Plattform übermittelt werden, nun aber zum Zweck der Weiterübermittlung (s. Kap. 1.6.4.1). Die weitere Verarbeitung erfolgt als Weiterübermittlung der Sozialdaten an abermals Dritte (s. Kap. 1.6.4.2).

\subsubsection{Szenario 3A (Weitergabe an und Auswertung durch die externe Plattform)}

\subsubsection{Zulässigkeit der Weitergabe durch den Leistungsträger (hinsichtlich der jeweiligen Zwecke) an die externe Plattform}

\subsection{Prüfungsmaßstab/Identifikation des einschlägigen Datenschutzregimes}

Hinsichtlich der Verarbeitungen durch die Krankenkassen bleibt es bei den in Kapitel 1.4.3.1 beschriebenen Rahmenbedingungen.

\subsection{Medizinische Forschung}

\section{Gesetzliche Ermächtigung}

Hinsichtlich einer Übermittlung von Sozialdaten zu Forschungszwecken, insbesondere zu medizinischen Forschungszwecken, kann im Wesentlichen auf die Ausführungen in Kapitel 1.5.3.2.4 verwiesen werden. Die Tatsache, dass die Daten verschiedener Krankenkassen bei der externen Plattform zusammengeführt werden, ist dann unproblematisch, wenn dieses Vorgehen vom jeweiligen Forschungsvorhaben und somit von der konkreten Zweckbestimmung im Einzelfall umfasst ist und folglich für die Durchführung des Forschungsvorhabens erforderlich ist.

Soll hingegen die externe Plattform Daten auf Vorrat speichern, die später ggf. zu Forschungszwecken verwendet werden könnten, so ist für eine Übermittlung keine Ermächtigungsgrundlage ersichtlich.

\section{Einwilligung}

Eine Übermittlung ist auf Crundlage einer Einwilligung zulässig. Hierzu kann auf die Ausführungen in Kapitel 1.5.3.3 verwiesen werden.

\section{Auftragsverarbeitung}

Eine Auftragsverarbeitung käme nur unter den in Kapitel 1.5.3.4 beschriebenen Voraussetzungen in Betracht. Zusätzlich ist darauf hinzuweisen, dass über eine Auftragsverarbeitung kein Datenumgang ermöglicht werden kann, der Daten anderer Krankenkassen betrifft. In einem solchen Fall würde der Auftragsverarbeiter, der dem Verantwortlichen zugerechnet werden muss, Daten 
über Versicherte anderer Leistungsträger (auch) für den Auftraggeber ggf. erheben, jedenfalls aber speichern. Diese Speicherung wäre nach $\mathbb{} 284 \mathrm{Abs}$. 1 SGB V unzulässig, da sie über die dort konkreten Daten hinausgehen würde. Weiterhin müsste die Weisungsbefugnis der Auftraggeber auch bei kollidierenden Weisungen der unterschiedlichen Auftraggeber wirksam werden können. Beide Anforderungen dürften bei der Arbeit mit Datenbeständen mehrerer Leistungsträger in jeweiliger Weisungsabhängigkeit praktisch nicht durchführbar sein, zumal der notwendige Konkretisierungsgrad der erforderlichen Verarbeitungsschritte regelmäßig nicht erreicht werden dürfte. Bestünden eigene Entscheidungsspielräume der externen Stelle hinsichtlich der Verarbeitungen, so wäre dies als Auftragsverarbeitung ohnehin nicht abbildbar.

\subsection{Weitere Zwecke}

Für die Übermittlung zu anderen Zwecken sind gemäß $₫ 67 \mathrm{~b}$ Abs. 1 SGB X entsprechende Übermittlungstatbestände im SGB zu identifizieren. Für die hier beispielhaft genannten übergeordneten Qualitätssicherungsaspekte ist eine solche Norm nicht ersichtlich. Alternativ kommt die Einholung einer Einwilligung nach den Vorgaben der DSGVO in Betracht (s. Kap. 1.4.3.3).

\subsubsection{Zulässigkeit der Auswertung durch die externe Plattform}

Die Auswertungsbefugnisse entsprechen den Darstellungen in Kapitel 1.5.4.

\subsubsection{Szenario 3B (Weitergabe an und Weiterübermittlung durch die externe Plattform)}

\subsubsection{Zulässigkeit der Übermittlung durch den Leistungsträger (hinsichtlich der jeweiligen Zwecke)}

\subsection{Prüfungsmaßstab/Identifikation des einschlägigen Datenschutzregimes}

Für eine Weiterübermittlung gilt das für die Verarbeitung durch eine externe Stelle anwendbare Datenschutzrecht, wie in Kapitel 1.5.4.1 dargestellt.

\subsection{Medizinische Forschung \\ Gesetzliche Ermächtigung}

Hinsichtlich einer Übermittlung von Sozialdaten zu Forschungszwecken, insbesondere zu medizinischen Forschungszwecken, kann auf die Ausführungen in Kapitel 1.6.3.1.2 verwiesen werden. Die Tatsache, dass die Daten verschiedener Krankenkassen bei der externen Plattform ggf. zusammengeführt werden, und anschließend weiterübermittelt werden sollen, ist dann unproblematisch, wenn dieses Vorgehen vom jeweiligen Forschungsvorhaben und somit von der konkreten Zweckbestimmung im Einzelfall umfasst ist und folglich für die Durchführung des Forschungsvorhabens erforderlich ist. 


\section{Auftragsverarbeitung}

Entsprechend $\$ 67 \mathrm{~d}$ Abs. 3 SGB X ist auch eine Übermittlung von Sozialdaten über Vermittlungsstellen im Rahmen einer Auftragsverarbeitung zulässig. Hierbei würde im Ergebnis eine Übermittlung von der Krankenkasse an die weitere dritte Stelle vorliegen, die nur durch die Plattform als Auftragsverarbeiter durchgeführt würde.

\section{Einwilligung}

Eine Übermittlung ist auf Crundlage einer Einwilligung zulässig. Hierzu kann auf die Ausführungen in Kapitel 1.5.3.3 verwiesen werden. Die Einwilligung muss sich auch auf die Weiterübermittlung beziehen.

\subsubsection{Zulässigkeit der Zusammenführung und Weitergabe durch die externe Plattform (hinsichtlich der jeweiligen Zwecke)}

Hinsichtlich einer Zusammenführung und Weiterübermittlung kommen dieselben Grundsätze, wie bei der Auswertung zum Tragen, da auch hier die absolute Zweckbindung nach $₫ 78$ Abs. 1 SGB X eingreift (s. Kap. 1.5.4). Wenn die vorherige Übermittlung also die Zwecke der Weiterübermittlung umfasst, ergibt sich für den Empfänger auch eine entsprechende Befugnis aus $₫ 78 \mathrm{Abs}$. 1 S. 1 SGB X. Die Wirkung des $\mathbb{7} 78$ SGB X perpetuiert sich anschließend auch gegenüber der weiteren Dritten.

\subsubsection{Anforderungen an „technische und organisatorische Maßnahmen“}

Die Anforderungen an technische und organisatorische Maßnahmen entsprechen denen der übermittelnden Stellen nach $₫ 35$ Abs. 1 SGB I.

\subsubsection{Genehmigungserfordernis und -anforderungen}

Hinsichtlich der Genehmigungsanforderungen ergeben sich keine abweichenden Besonderheiten zu den in Kapitel 1.5.4.6 dargestellten Grundsätzen.

\subsection{Sozialrechtliche Zulässigkeit der Zusammenführung von Sozialdaten mit weiteren Patientendaten}

\subsubsection{Ohne Einbindung eines Treuhänders oder einer Vertrauensstelle}

Bei der Frage, ob Krankenkassen Sozialdaten mit weiteren Patientendaten zusammenführen dürfen, ist der streng zu verstehende $\$ 284$ Abs. 1 SGB V zu beachten. Dieser bestimmt abschließend, zu welchen Zwecken Krankenkassen 
Sozialdaten erheben und speichern dürfen. Eine Erhebung zu Forschungszwecken ist hiervon nicht umfasst.

Denkbar wäre allenfalls die Legitimation über die Einholung einer Einwilligung nach der DSGVO. Hinsichtlich - hier maßgeblich in Rede stehender Gesundheitsdaten besteht nach Art. 9 Abs. 2 lit. a) DSGVO aber die Möglichkeit der Mitgliedsstaaten, die Einwilligung auszuschließen. Es wäre daher möglich, $\mathbb{2} 284$ Abs. 1 SGB V derart zu interpretieren, dass eine abweichende Zweckbestimmung auch durch eine Einwilligung nicht möglich sein soll.

\subsubsection{Mit Einbindung eines Treuhänders oder einer Vertrauensstelle}

Ohne eine eigenständige rechtliche Grundlage, die die Einbindung weiterer Stellen in der Funktion eines Datentreuhänders oder einer Vertrauensstelle ermöglicht, führt die Einbindung einer solchen Stelle nicht zu einer abweichenden Bewertung. Zwar wäre es sowohl aus grundrechtlicher Perspektive vertretbar, wie auch aus Gründen der Praktikabilität wünschenswert, eine gesonderte Stelle zu involvieren, die in einheitlichen, validierten Verfahren Anonymisierungsprozesse vornimmt und überwacht, sowie die Speicherung der Daten unter besonders hohen Anforderungen der Datensicherheit sicherstellen kann. Allerdings müsste eben diese Stelle - wie auch jede andere Stelle - zur Verarbeitung personenbezogener Daten befugt sein.

\subsubsection{Erstreckung der sozialrechtlich begründeten Genehmigung auf die Rechtmäßigkeit der Verwendung weiterer Gesundheitsdaten}

Existiert eine sozialrechtliche Genehmigung für die Verarbeitung personenbezogener Daten, etwa nach $₫ 75$ Abs. 4 SGB X für die Verarbeitung, umfasst diese grundsätzlich Verarbeitungsvorgänge bezüglich der in der Cenehmigung benannten (Sozial-)Daten. Sollen auch andere Gesundheitsdaten - etwa von medizinischen Leistungserbringern (z.B. Ärzte, Krankenhäuser) - verarbeitet werden, richtet sich die Zulässigkeit der Verarbeitung dieser Daten nach den jeweils geltenden Normen und Erlaubnistatbeständen. Die sozialrechtliche Genehmigung kann diese nicht ohne Weiteres erfassen, auch wenn der Sinn und Zweck des Vorhabens eine solche Verarbeitung weiterer Daten voraussetzt. Dafür wäre eine gesonderte gesetzliche Anordnung erforderlich, die eine solche Erstreckungswirkung im Einzelfall vorsieht. Dies geht bereits aus $\mathbb{5} 67 \mathrm{~d}$ Abs. 2 SGB X hervor, der regelt, dass überschießende, also für das konkrete Vorhaben nicht benötigte Daten zwar übermittelt, nicht jedoch verarbeitet werden dürfen. Wenn eine solche Beschränkung bereits für Sozialdaten des gleichen Leistungsträgers gilt, kann eine Erstreckung auf andere Bereiche nicht ohne Weiteres möglich sein. 


\subsection{Löschverpflichtungen in den jeweiligen Szenarien \\ 1.8.1 Verlängerte Aufbewahrung zum Zweck der Nachvollziehbarkeit der Auswertungen}

Nach dem Grundsatz der Datenminimierung (Art. 5 lit. c) DSGVO) gilt, dass personenbezogene Daten zu löschen sind, wenn sie nicht mehr für den Zweck, für den sie erhoben oder sonst verarbeitet wurden, benötigt werden. Diesen Grundsatz greift Art. 17 Abs. 1 lit. a) DSGVO auf und statuiert eine entsprechende Löschverpflichtung des Verantwortlichen. Demnach wären die zu Forschungs- und Planungszwecken übermittelten Sozialdaten mit Abschluss des Forschungsvorhabens grundsätzlich zu löschen. Art. 17 Abs. 3 lit. b), c) und d) DSGVO regeln jedoch Ausnahmen von diesem Grundsatz, sofern die Verarbeitung zur Erfüllung einer rechtlichen Verpflichtung, der Wahrnehmung einer im öffentlichen Interesse liegenden Aufgabe oder in Ausübung der öffentlichen Gewalt (lit. b), aus Gründen des öffentlichen Interesses im Bereich der Gesundheit (lit. c) oder für im öffentlichen Interesse liegende Forschungszwecke erforderlich ist. Mit diesen Ausnahmeregelungen korrespondieren die Öffnungsklauseln der Art. 6 Abs. 1 lit. c), e) sowie Art. 9 Abs. 2 lit. h) und i) DSGVO, wodurch die nationalen Gesetzgeber die Möglichkeit erhalten, konkretisierende Vorschriften zu erlassen. ${ }^{219}$

Für den Bereich des nationalen Sozialdatenschutzes bestimmt $\$ 304$ Abs . 1 S. 1 SGB V unter Verweis auf $\mathbb{} 84$ Abs. 2 SGB X a.F., dass Sozialdaten, die bei den Krankenkassen, Kassenärztlichen Vereinigungen und Geschäftsstellen der Prüfungsausschüsse gespeichert sind, zu löschen sind, wenn ihre Speicherung unzulässig ist oder ihre Kenntnis für die rechtmäßige Erfüllung der Aufgaben der verantwortlichen Stelle nicht mehr erforderlich ist und kein Grund zu der Annahme besteht, dass durch die Löschung schutzwürdige Interessen des Betroffenen beeinträchtigt werden. Darüber hinaus legt $\$ 304$ Abs. 1 SGB V Höchstfristen fest, nach denen die genannten Daten in jedem Fall zu löschen sind, sofern sie im Einzelfall mit entsprechender Rechtsgrundlage über den nach $\mathbb{5} 84$ Abs. 2 SGB X bestimmten Zeitraum gespeichert werden. Vor dem Hintergrund der unmittelbaren Regelung durch Art. 17 DSGVO und des daran angepassten $\$ 84$ SGB X ergibt sich Änderungsbedarf für $\$ 304$ SGB V, dessen Inbezugnahme des $\mathbb{5} 84$ Abs. 2 SGB X a.F. ab dem 25.05.2018 obsolet sein wird.

Für den Bereich der wissenschaftlichen Forschung und Planung enthält $\mathbb{} 75$ SGB X eine Sondervorschrift für die Aufbewahrung der zu Forschungs- und Planungszwecken i.S.d. $\$ 75$ Abs. 1 SCB X an eine externe Einrichtung übermittelten Daten. So besagt $\$ 75$ Abs. 4 S. 6 SGB X, dass die zu Forschungs- und Planungszwecken i.S.d. $\mathbb{S} 75$ Abs. 1 SGB X übermittelten und verarbeiteten Daten bis zu 10 Jahre über den Zeitpunkt, den die Genehmigungsbehörde für die zulässige Verarbeitung nach $\mathbb{7} 75$ Abs. 4 S. 5 Nr. 4 SGB X festgelegt hat, hi-

219 Herbst, in: Kühling/Buchner, DS-GV0, 2017, Art. 17 Rn. 74, 79. 
naus gespeichert werden dürfen, um eine Nachprüfung der Forschungsergebnisse sowie eine weitere Verarbeitung für Forschungsfolgefragen nach $\mathbb{} 75$ Abs. 2 SGB X zu ermöglichen.

Es obliegt der Genehmigungsbehörde, durch Auflagen zu bestimmen, wie lang und bei welcher Stelle (übermittelnde oder empfangende Stelle) die Daten gespeichert werden können. ${ }^{220}$ Zudem kann sie weitere Auflagen für die verlängerte Speicherung erteilen. Eine längere Verarbeitung im Sinne der bloßen Aufbewahrung ist hierüber gerechtfertigt. Die Verarbeitung im Rahmen der konkreten Nachprüfung oder weiteren Forschungsfrage wird sich in aller Regel auf dieselben Rechtmäßigkeitstatbestände stützen können wie das ursprüngliche Forschungsvorhaben und bedürfte ggf. einer eigenen Genehmigung. Kommt es nicht zu einer Nachprüfung oder Forschungsfolgefrage, wären die Daten zu löschen. Werden sie hingegen zu weiteren Forschungsfragen herangezogen, spricht viel dafür, die Daten auch zur Nachprüfung der dadurch erreichten Forschungsergebnisse erneut 10 Jahre aufbewahren zu können.

Ohne eine entsprechende Genehmigung oder nach Ablauf der in der Genehmigung festgelegten Frist, sind die zu Forschungszwecken übermittelten Daten zu löschen, sofern sie für das konkrete Forschungsvorhaben oder eine bereits bestehende Folgefrage nach $\$ 75$ Abs. 2 SGB X nicht mehr erforderlich sind.

\$ 84 SGB X regelt zudem Einschränkungen der Löschverpflichtungen. Gemäß $\$ 84$ Abs. 1 SGB X kann bei einer nicht-automatisierten Verarbeitung von einer Löschung abgesehen werden, wenn die Löschung auf Grund der Art der Speicherung nicht oder nur mit unverhältnismäßigem Aufwand möglich, das Interesse des Betroffenen an der Löschung gering ist und die Verarbeitung rechtmäßig war. Stattdessen ist die Verarbeitung dieser Daten gemäß Art. 18 DSGVO einzuschränken und somit die Daten für eine weitere Verarbeitung zu sperren. Eine Sperrung der Daten ist nach $\$ 84$ Abs. 4 SGB X auch erforderlich, sofern der Löschung nach dem Wegfall des Zwecks satzungsmäßige oder vertragliche Aufbewahrungsfristen entgegenstehen.

Somit bleibt für Szenario 1 festzuhalten, dass die Krankenkassen die bei ihnen gespeicherten Sozialdaten (bisher) gemäß $\$ 304$ Abs. 1 SGB V a.F. bzw. (zukünftig) gemäß Art. 17 DSGVO i.V.m. $\mathbb{8} 84$ SGB X (ggf. i.V.m. einem neuen $\mathbb{3} 304$ SGB V) unverzüglich zu löschen haben, wenn diese Daten nicht mehr für die Erfüllung der ihnen übertragenen Aufgaben erforderlich sind. Dürfen die Daten auf Grund einer Rechtsvorschrift länger aufbewahrt werden, sind die Daten spätestens mit Ablauf der Höchstfrist zu löschen. Es spräche im Fall des

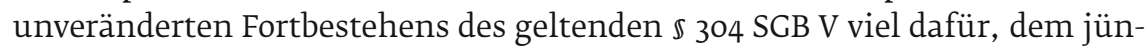
geren Recht des es neuen $\mathbb{5} 75$ SGB V Vorrang zu gewähren und im Falle der Verarbeitung von Sozialdaten zu Forschungszwecken die Höchstfristen des \$ 304 SGB V nicht anzuwenden.

220 BT-Drs. 18/12611, S. 119. 
Für die Szenarien 2 und 3 bestimmt $\$ 75$ Abs . 4 S. 6 SGB X darüber hinaus, dass die zu Forschungs- und Planungszwecken entsprechend der Festlegungen der Genehmigungsbehörde über den für das Forschungsvorhaben selbst festgelegten Zeitpunkt hinaus 1o Jahre aufbewahrt werden können, um zum Zwecke der Nachprüfung der Forschungsergebnisse sowie der Bearbeitung von Forschungsfolgefragen verarbeitet werden zu können.

\subsubsection{Anwendbarkeit des $\S 304$ SGB V auf eine externe Einrichtung}

Auch die externe Einrichtung hat die Daten gemäß Art. 17 DSGVO grundsätzlich zu löschen, sobald deren Verarbeitung für den jeweiligen Zweck nicht mehr erforderlich ist. Sofern die externe Stelle die Daten gemäß $₫ 75$ SGB X von einem Leistungsträger erhalten hat, kommt eine weitergehende Aufbewahrung der Daten zum Zwecke der Nachprüfung der Forschungsergebnisse sowie der Bearbeitung von Forschungsfolgefragen in Betracht, soweit und solange die gemäß $\$ 75$ Abs. 4 SGB X zuständige Behörde die Aufbewahrung durch die externe Stelle genehmigt hat.

Eine Übertragung der allgemeinen Aufbewahrungsfristen nach $\mathbb{3} 304$ SGB V ergibt sich in diesem Zusammenhang jedoch nicht. Zwar ist die externe Stelle gemäß $\$ 78$ Abs. 1S. 3 SGB X gleichermaßen zur Geheimhaltung nach $\sqrt{35}$ SGB I wie der verantwortliche Leistungsträger verpflichtet. Daraus folgt jedoch nicht, dass alle für diese geltenden Regelungen gleichermaßen auf die externe Stelle zu übertragen sind. $\$ 304$ SGB V sichert die begrenzte Speicherung von Sozialdaten durch die Krankenkassen ab. Die Kassen sollen lediglich die Daten aufbewahren dürfen, die sie zur Erfüllung der ihnen übertragenen Aufgaben benötigen. Alle anderen Daten sind nach der Zweckerreichung zu löschen. In diesem Sinn ist es nicht erforderlich, auch eine externe Stelle den Vorgaben des $\mathbb{3} 304$ SGB V zu unterwerfen. Vielmehr ist die externe Stelle gemäß Art. 17 Abs. 1 DSGVO i.V.m. $\mathbb{S} 84$ SGB X verpflichtet, die ihr übermittelten Daten nach der Erreichung des Zwecks zu löschen, sofern nicht eine gesetzliche Vorschrift -

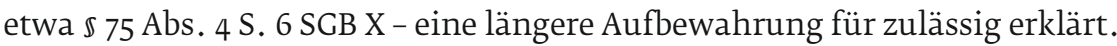

\subsubsection{Erfüllung der löschverpflichtung durch Anonymisierung}

Das deutsche Datenschutzrecht definierte bisher das Löschen als das „Unkenntlichmachen gespeicherter personenbezogener Daten“ (vgl. $\$ 3$ Abs. 4 Nr. 5 BDSG a.F., $\mathbb{s} 67$ Abs. 4 S. 2 Nr. 5 SGB X a.F.). Hierzu war anerkannt, dass eine Löschung durch eine physische Vernichtung oder auch durch sicheres Überschreiben erfolgen kann. ${ }^{221}$ Mit der Löschung der Daten entfällt die Anwendbarkeit des Datenschutzrechts. Die gleiche Wirkung kann mit einer An- 
onymisierung erreicht werden. ${ }^{222}$ Es stellt sich daher die Frage, ob ein Löschanspruch gleichermaßen mit einer Anonymisierung von Daten erfüllt werden kann. Die Anonymisierung hätte den großen Vorteil für den Verantwortlichen, dass die nach erfolgreicher Anonymisierung verbleibenden (nicht mehr personenbezogenen) Einzelangaben, beispielsweise zum Zweck statistischer Auswertungen, weiterverwendet werden könnten. Ihnen kommt somit unter Umständen weiterhin ein erheblicher Wert zu, der bei einer vollständigen Löschung verloren gehen würde. Gleichzeitig endet aber auch die Betroffenheit der informationellen Selbstbestimmung mit Entfall des Personenbezugs.

Bereits nach alter Rechtslage war daher wohl anerkannt, dass auch die Anonymisierung von Daten eine Form der Löschung darstellen kann. ${ }^{223}$ Dies ergibt sich nicht unmittelbar aus dem Wortlaut des Gesetzes, doch Sinn und Zweck der Vorschrift sprechen dafür, da eine Anonymisierung die vollständige Aufhebung des Personenbezugs darstellt. ${ }^{224}$ Die herrschende Meinung ging davon aus, dass der Betroffene nach $\mathbb{3} 35$ BDSG a.F. statt der Löschung erst recht eine Anonymisierung oder Pseudonymisierung verlangen konnte. 225 Technisch muss insgesamt ein Datenbestand geschaffen werden, der die personenbezogenen Daten nicht mehr enthält. ${ }^{226}$ Eine getrennte Speicherung reicht nicht aus. ${ }^{227}$

Die DSGVO enthält keine Legaldefinition der Löschung. Neben dem Begriff der Löschung nennt die DSGVO aber auch den der Vernichtung als Unterfall der Verarbeitung der ebenfalls in Art. 4 Nr. 2 DSGVO. Aus dieser Unterscheidung lässt sich ableiten, dass eine Löschung nicht zwingend eine Vernichtung voraussetzt. ${ }^{228}$ Es sind jedoch keine Anhaltspunkte ersichtlich, aus denen sich eine von vom bisherigen Verständnis abweichende Definition ergeben könnte. Hinsichtlich der Mittel und Verfahren der Löschung steht dem verantwortlichen ein Auswahlermessen zu. ${ }^{229}$ Die Wirkung der Anonymisierung, wird, wie oben dargestellt, unverändert auch unter DSGVO gelten. Daher kann sie nach hier vertretener Ansicht auch zukünftig ein geeignetes Mittel sein, um einen Löschanspruch zu erfüllen. Art. 17 Abs. 2 DSGVO enthält im Rahmen des „Rechts auf Vergessenwerden“ den Hinweis, dass alle „Kopien oder Replikationen" von einem Löschungsersuchen umfasst sind. Daher gilt auch weiterhin, dass alle Kopien zu löschen oder zu anonymisieren sind. Das folgt auch unmittelbar aus den Erwägungen zur Wirksamkeit der Anonymisierung. ${ }^{230}$

222 Siehe hierzu die Ausführungen im Gutachtenteil von Roßnagel im vorliegenden Band.

223 BeckOK DatenSR/Brink, 20. Ed. 1.2.2017, BDSG $§ 35$ Rn. 26, beck-online; Dix, in: Simitis, BDSG, 8. Aufl., 2014, $§ 35$ Rn. 45, Plath/Schreiber, in: Plath, BDSG/DSGVO, 2. Aufl., 2016, § 3 BDSG Rn. 52.

224 Dix, in: Simitis, BDSG, 8. Aufl., 2014, \35 Rn. 45.

225 Meents/Hinzpeter, in: Taeger/Gabel, BDSG, 2. Aufl., 2013, §35 Rn. 17; Dix, in: Simitis, BDSG, 8. Aufl., 2014, §35 Rn. 45.

226 BeckOK DatenSR/Schild BDSG, 20. Ed. 1.5.2017, \$ 3 Rn. 98, beck-online.

227 Greve, in: Auerhammer, DSGVO/BDSG, 5. Aufl., 2017, $\$ 40$ BDSG Rn. 14.

228 Kamann/Braun, in: Ehmann/Selmayr, DS-GVO, 2017, Art. 17 Rn. 32 (m.w.N.).

229 Kamann/Braun, in: Ehmann/Selmayr, DS-GVO, 2017, Art. 17 Rn. 36.

$230 \mathrm{Vgl}$ Klabunde, in: Ehmann/Selmayr, DS-GVO, 2017, Art. 4 Rn. 16. 
Aus der deutschen Anpassungsgesetzgebung ergibt sich keine abweichende Erwägung.

Nach hier vertretender Auffassung kommt eine Anonymisierung, bei der alle Kopien der Datensätze ebenfalls anonymisiert werden, daher einer Löschung gleich.

\subsection{Verpflichtung externer Einrichtungen zur Übermittlung von Sozialdaten an weitere Sozialleistungsträger ( $\$$ 18-29 SGB I)}

Sofern die Übermittlung von Sozialdaten an eine externe Stelle nach den oben dargelegten Crundsätzen zulässig ist, ist die Verarbeitung dieser Daten durch die externe Stelle durch den Zweckbindungsgrundsatz streng limitiert (vgl. $\mathbb{\$} 78$ Abs. 1 SGB X). Eine Datenverarbeitung der externen Stelle, zu der auch die Datenübermittlung gehört, darf nur erfolgen, sofern sie von dem Zweck gedeckt ist, zu dem ihr die Daten übermittelt wurden. Eine Übermittlung an andere Sozialleistungsträger ist mithin nur zulässig, wenn sich die Übermittlung im Rahmen des Zweckes bewegt, zu dem die Daten der externen Stelle übertragen wurden oder wenn eine anderweitige gesetzliche Ermächtigungsgrundlage für die Übermittlung existiert. Eine grundsätzliche Verpflichtung, erhaltene Sozialdaten an andere Sozialleistungsträger zu übermitteln, auch wenn diese die Daten für die ihnen zugewiesenen Zwecke verarbeiten, existiert nicht.

Sofern für die übermittelnde Stelle eine Verpflichtung besteht, Sozialdaten an bestimmte andere Leistungsträger zu übermitteln, gehen diese Übermittlungspflichten des Leistungserbringers nicht ohne Weiteres mit der Datenübermittlung auf die externe Stelle über. Vielmehr verbleibt die Pflicht beim Leistungsträger. Begehrt ein anderer Sozialleistungsträger personenbezogene Sozialdaten, muss er sich, sofern er nicht ohnehin zur Erhebung der Daten beim Betroffenen selbst verpflichtet ist ( $\$ 67 a$ Abs. 2 S. 1 SGB X), an den Leistungsträger wenden, der entsprechend der gesetzlichen Vorschriften zur Übermittlung der begehrten Daten verpflichtet ist. Nur sofern im Einzelfall eine gesetzliche Vorschrift existiert, die - neben dem Übergang des Sozialgeheimnisses (z.B. $\mathbb{S} 78$ Abs. 1 S. 3 SGB X) - den Übergang der Übermittlungspflichten anordnet, kann der Empfänger zur Übermittlung der erhaltenen Sozialdaten verpflichtet sein.

Auch $\sqrt{5} 69$ SGB X statuiert keine Übermittlungsverpflichtung für die externen Stellen. $\mathbb{5} 69$ SGB X berechtigt lediglich die in $\mathbb{3} 35$ Abs . 1 SGB I genannten sowie die ihnen nach $\$$ g Abs. 2 SGB X gleichgestellten Stellen zur Übermittlung von Sozialdaten. Diese Übermittlungsbefugnis kann sich allenfalls zu einer Übermittlungspflicht verdichten, wenn diese Stelle zur Amtshilfe verpflichtet ist oder die Übermittlung selbst zu ihren gesetzlichen Aufgaben gehört. ${ }^{231}$ Exter-

231 Rombach, in: Hauck/Noftz, SGB X, Stand: 08/2017, \$ 69 Rn. 79. 
ne Stellen, die keine in $\mathbb{3} 35 \mathrm{Abs}$. 1 SGB I genannte oder dieser gleichgestellten Stelle sind, können folglich keiner Übermittlungspflicht nach $\mathbb{s} 69$ SGB X unterliegen. Handelt es sich bei der empfangenden Stelle um eine in $\$ 35 \mathrm{Abs}$. 1 SGB I genannte oder diesen gleichgestellte Stelle, ist zu bedenken, dass der andere Sozialleistungsträger grundsätzlich verpflichtet ist, die Daten bei der Stelle anzufragen, die die Daten ursprünglich an die externe Stelle übermittelt hat (Direkterhebung gemäß 5 67a Abs. 2 S. 1 SGB X). Nur soweit der Übergang einer solchen Verpflichtung auf den Empfänger der Sozialdaten vom Gesetz ausdrücklich angeordnet wird oder die Daten vom Verantwortlichen nicht mehr übermittelt werden können, kommt eine Pflicht zur Übermittlung durch die externe Stelle in Betracht.

Handelt es sich bei der externen Stelle um einen Auftragsverarbeiter ist die Verarbeitungsbefugnis durch den Auftrag und die Weisungen des Verantwortlichen sowie dessen Verarbeitungsbefugnisse begrenzt. Dementsprechend kann sich für die externe Stelle eine Übermittlungspflicht ergeben, sofern der Verantwortliche selbst zur Übermittlung verpflichtet ist und der Auftrag etwa die Speicherung und Übermittlung dieser Daten umfasst. Betrifft der Auftrag jedoch andere Tätigkeiten, schließt dies die Übermittlung durch den Auftragsverarbeiter jedoch aus. Zudem unterliegt der Auftragsverarbeiter den Weisungen des Verantwortlichen. Folglich ist eine eigenmächtige Übermittlung der Daten durch den Auftragsverarbeiter in jedem Fall unzulässig. Vielmehr bedarf es einer entsprechenden Weisung durch den Auftraggeber.

\subsection{Auskunftsrechte der Versicherten}

Das Auskunftsrecht des Betroffenen ist in Art. 15 DSGVO geregelt. $\mathbb{S} 34$ BDSG und $\$ 83$ SGB X erhalten dazu ergänzende Regelungen, die den Anspruch im Wesentlichen einschränken und weitere Voraussetzungen formulieren. ${ }^{232} \mathrm{Da}$ es sich bei den von der Krankenkasse erhobenen Daten um Sozialdaten im

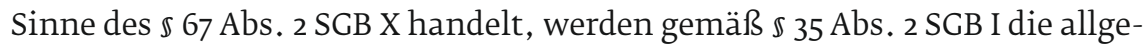
meinen datenschutzrechtlichen Anforderungen des $\mathbb{S} 34$ BDSG von den spezielleren sozialrechtlichen Bestimmungen des $\$ 83$ SGB X verdrängt.

Ob dies auch im Falle der Übermittlung der Daten an die externe Stelle gilt (insbesondere in Szenario 3), ergibt sich nicht eindeutig aus dem Gesetz. Es spricht aber viel dafür, dass sich der Auskunftsanspruch gegenüber einer externen Stelle, gegenüber der sich das Sozialgeheimnis nach $\$ 78$ Abs. 1 SGB X verlängert, nach den gleichen Anforderungen richtet, wie dies gegenüber einer Stelle nach $\mathbb{3} 35$ Abs. 1 SGB X der Fall wäre. Dies ergäbe sich im Übrigen auch aus $\mathbb{S} 35$ Abs. 6 S. 1 Nr. 1 SGB I, sofern man diese Regelung nicht unter Berücksichtigung des $\mathbb{5} 78 \mathrm{Abs}$. 1 SGB X restriktiv auslegen würde. 


\subsubsection{Auskunftsanspruch gegen die gesetzliche Krankenkasse}

Der Auskunftsanspruch besteht gegenüber dem Verantwortlichen. Dies ist gemäß Art. 4 Nr. 7 DSGVO diejenige natürliche oder juristische Person, die die Entscheidungsgewalt über die personenbezogenen Daten hat. In allen drei Szenarien handelt es sich dabei um die gesetzliche Krankenkasse. Diese erhebt die Sozialdaten beim Betroffenen und übermittelt sie ggf. an eine externe Stelle.

\subsubsection{Voraussetzungen des Auskunftsanspruchs}

Art. 15 DSGVO enthält keine besonderen Anforderungen für die Geltendmachung des Auskunftsanspruchs. Der Versicherte kann seinen Antrag daher formlos, ggf. sogar mündlich stellen. Er hat jedoch seine Identität nachzuweisen, vgl. Art. 12 Abs. 1 und 2 DSGVO. Bei Zweifeln hinsichtlich der Identität kann die verantwortliche Stelle zusätzliche Informationen verlangen (Art. 12 Abs. 6 DSGVO). Gemäß $\mathbb{5} 83$ Abs. 2 S. 1 SGB X soll der Betroffene in seinem Antrag die Art der Sozialdaten, über die er Auskunft begehrt, näher bezeichnen. Pauschale Auskunftsersuchen können daher von der Krankenkasse mit der Bitte um Spezifizierung zurückgewiesen werden.

\subsubsection{Umfang des Auskunftsanspruchs}

Gemäß Art. 15 DSGVO hat der Betroffene gegenüber dem Verantwortlichen Anspruch auf Auskunft darüber, ob und in welchem Umfang personenbezogene Daten von ihm verarbeitet werden. Aus Erw.Gr. 63 der Verordnung ergibt sich, dass sich der Auskunftsanspruch auch auf eigene gesundheitsbezogene Daten (beispielsweise Daten in Patientenakten, Untersuchungsergebnisse oder Befunde) bezieht.

Er kann im Einzelnen Auskunft verlangen über (vgl. Art. 15 Abs. 1, Abs. 2 DSGVO):

- die Verarbeitungszwecke

- die Kategorien personenbezogener Daten, die verarbeitet werden

- falls möglich die geplante Dauer, für die die personenbezogenen Daten gespeichert werden, oder, falls dies nicht möglich ist, die Kriterien für die Festlegung dieser Dauer

- das Bestehen eines Rechts auf Berichtigung oder Löschung der sie betreffenden personenbezogenen Daten oder auf Einschränkung der Verarbeitung durch den Verantwortlichen oder eines Widerspruchsrechts gegen diese Verarbeitung ${ }^{233}$

- das Bestehen eines Beschwerderechts bei einer Aufsichtsbehörde ${ }^{234}$

233 Vgl. Art. 16 und 17 DS-GVO.

234 Vgl. §81 Abs. 1 SGB X. 
- wenn die personenbezogenen Daten nicht bei der betroffenen Person erhoben werden ${ }^{235}$, alle verfügbaren Informationen über die Herkunft der Daten

- das Bestehen einer automatisierten Entscheidungsfindung einschließlich des Profilings gemäß Artikel 22 Absätze 1 und 4 DSGVO und - zumindest in diesen Fällen - aussagekräftige Informationen über die involvierte Logik sowie die Tragweite und die angestrebten Auswirkungen einer derartigen Verarbeitung für die betroffene Person

- die geeigneten Garantien gemäß Artikel 46 DSGVO im Falle der Übermittlung der Daten an ein Drittland oder an eine internationale Organisation.

- die Empfänger oder Kategorien von Empfängern, gegenüber denen die personenbezogenen Daten offengelegt worden sind oder noch offengelegt werden, insbesondere bei Empfängern in Drittländern oder bei internationalen Organisationen.

Gemäß $\$ 83$ Abs. 1 Nr. 1 i.V.m. $\mathbb{8} 82$ a Abs. 4 i.V.m. $\$ 82$ Abs. 1 SGB X sind hier nur diejenigen Empfänger zu nennen, an die eine Weiterleitung aus Sicht des Betroffenen nicht zu erwarten ist, die nicht zu den in $\mathbb{} 35$ SGB I genannten Personen oder Stellen gehören oder eng mit diesen zusammenarbeiten.

Zu nennen sind dagegen in Szenario 2 und $\mathbf{3}$ die externe Stelle, in Szenario 3 ggf. weitere Dritte. Die Drittempfänger sollten dabei soweit wie möglich präzisiert werden. Bei der Benennung von Empfängerkategorien reichen die üblichen Branchenbezeichnungen aus. ${ }^{236}$

\subsubsection{Verfahren und Form}

Art. 12 DSGVO enthält verschiedene Anforderungen an die Auskunftserteilung. Sie muss in präziser, transparenter und leicht zugänglicher Form erteilt und in klarer und einfacher Sprache verfasst werden (Art. 12 Abs. 1 DSGVO). In der Regel ist die Auskunft schriftlich oder elektronisch zu übermitteln, kann aber auf Verlangen des Versicherten auch mündlich erfolgen. Einzelheiten zum Verfahren, insbesondere zur Form der Auskunft, kann die Krankenkasse bestimmen ( 83 Abs. 2 S. 3 SGB X). Soweit die Daten Angaben zu gesundheitlichen Verhältnissen des Betroffenen enthalten, kann sie die Auskunft gemäß $\mathbb{5} 83$ Abs. 2 S. 4 i.V.m. $\mathbb{2} 25$ Abs. 2 SGB X durch einen Arzt erteilen lassen.

Art. 12 Abs. 3 DSGVO sieht für die Erteilung der Auskunft eine Frist von einem Monat vor. Diese Frist kann um maximal zwei Monate verlängert werden. Auch darüber - sowie über die Cründe einer solchen Fristverlängerung - ist der Betroffene in Kenntnis zu setzen. 
Gemäß Art. 15 Abs. 3 DSGVO muss die Krankenkasse dem Betroffenen eine Kopie der verarbeiteten personenbezogenen Daten zur Verfügung stellen (im Falle der elektronischen Übermittlung kann dies z.B. im PDF-Format erfolgen). Diese Kopie sowie die Auskunftserteilung selbst erfolgt unentgeltlich. Nur für weitere Kopien kann die Krankenkasse ein angemessenes Entgelt verlangen.

\subsubsection{Ausschluss des Anspruchs}

Gemäß $\mathbb{} 83$ Abs. 1 Nr. 1 SGB X besteht kein Auskunftsanspruch, wenn der Betroffene gemäß $\mathbb{8} 82 \mathrm{a}$ SGB X nicht zu informieren ist, die Auskunftserteilung einen unverhältnismäßigen Aufwand erfordern würde und eine Verarbeitung zu anderen Zwecken durch geeignete technische oder organisatorische Maßnahmen ausgeschlossen ist. Dies ist gemäß $\mathbb{S} 82 \mathrm{~A}$ Abs. 1 SGB X insbesondere dann der Fall, wenn die ordnungsgemäße Erfüllung der Aufgaben des Verantwortlichen oder die öffentliche Sicherheit und Ordnung gefährdet wäre oder wenn die Daten ihrem Wesen nach oder aufgrund einer gesetzlichen Regelung geheim gehalten werden müssen und deswegen das Interesse des Betroffenen an der Erteilung der Auskunft zurücktreten muss. Damit werden die Bestimmungen des bisherigen $\$ 83$ Abs. 2 und Abs. 4 SGB X a.F. beibehalten. ${ }^{237}$ Die Beschränkung des Auskunftsanspruchs soll die verantwortliche Stelle insbesondere vor einer übermäßigen Inanspruchnahme schützen. ${ }^{23^{8}}$ Ein vollständiger Ausschluss des Auskunftsanspruchs dürfte jedoch nur im Ausnahmefall möglich sein. Das Auskunftsersuchen kann beispielsweise nicht bei bloßer Arbeitsüberlastung abgelehnt werden. ${ }^{239}$ Stattdessen kann der Verantwortliche verlangen, dass die betroffene Person präzisiert, auf welche Information oder welche Verarbeitungsvorgänge sich ihr Auskunftsersuchen bezieht. ${ }^{240}$

Die Auskunft kann ferner verweigert werden, wenn die Sozialdaten nur noch gespeichert sind, weil sie aufgrund gesetzlicher oder satzungsmäßiger Aufbewahrungsvorschriften nicht gelöscht werden dürfen ( $\$ 83$ Abs. 1 Nr. 2 lit. a SCB X) oder wenn sie nur der Datensicherung oder -kontrolle dienen ( $\$ 83 \mathrm{Abs} .1$ Nr. 2 lit. b SGB X), und die Auskunftserteilung einen unverhältnismäßigen Aufwand erfordern würde und eine Verarbeitung zu anderen Zwecken durch geeignete technische oder organisatorische Maßnahmen ausgeschlossen ist. Bei den archivierten ${ }^{241}$ oder zu Kontroll- und Sicherheitszwecken gespeicherten $^{242}$ Daten ist dabei zu beachten, dass der Verantwortliche ggf. technische Maßnahmen ergreifen muss, um die Daten verfügbar zu machen. ${ }^{243}$

\footnotetext{
237 BT-Drucks. 18/12611, S. 129.

238 BT-Drucks. 18/12611, S. 130.

239 Diering/Seidel, in: Diering/Timme, SGB X, 4. Aufl., 2016, \$ 83 Rn. 7.

240 Vgl. Erw.Gr. Nr. 63 S. 7, siehe auch $\$ 83$ Abs. 2 S. 1 SGB X.

$241 \mathrm{Vgl}$ \$84 Abs. 3 SGB X.

242 Vgl. \$ 67c Abs. 4 SGB X.

243 BT-Drucks. 18/12611, S. 130.
} 
Bei nicht automatisiert oder in nicht automatisierten Dateisystemen gespeicherten Daten muss die Auskunft gemäß $₫ 83$ Abs. 2 S. 2 SGB X ferner nur dann erteilt werden, wenn der Betroffene Angaben macht, die ein Auffinden der Daten ermöglichen und die Erteilung der Auskunft keinen unverhältnismäßigen Aufwand erfordert. ${ }^{244}$ Hierfür reicht die Mitteilung des jeweiligen Aktenzeichens aus. ${ }^{245}$

Abgesehen davon kann die verantwortliche Stelle gemäß Art. 12 Abs. 5 DSGVO die Auskunft verweigern, wenn entsprechende Anträge offenkundig unbegründet sind oder exzessiv gestellt werden. Der Antrag ist „offenkundig unbegründet“, wenn seine Voraussetzungen offensichtlich nicht erfüllt sind, z.B. wenn der Anspruch nicht von dem Betroffenen selbst geltend gemacht wird. ${ }^{246}$ Eine „exzessive“ Antragstellung dürfte nur selten vorliegen. Dem Erw. Gr. 63 S. 1 lässt sich entnehmen, dass der Auskunftsanspruch in ,angemessenen Abständen " geltend gemacht werden kann. Wann eine exzessive Antragstellung vorliegt, ist eine Frage des Einzelfalls. Es sollte dem Betroffenen jedoch möglich sein, das Auskunftsrecht mehrmals pro Jahr geltend zu machen.

Von den genannten Verweigerungsrechten kann die Krankenkasse sowohl bei Datenerhebungen aufgrund einer gesetzlichen Ermächtigung als auch bei solchen auf Grundlage von Einwilligungen Gebrauch machen. Gemäß $\mathbb{} 83$ Abs. 3 S. 1 SGB X hat sie die Gründe für die Auskunftsverweigerung zu dokumentieren, es sei denn, der mit der Auskunftsverweigerung verfolgte Zweck würde dadurch gefährdet werden (z.B. wenn die Dokumentation in Rechte Dritter eingreifen würde).

\subsubsection{Auskunftsanspruch gegen die externe Stelle}

\subsubsection{Auftragsverarbeiter}

Ist die externe Stelle nur „Auftragsverarbeiter“ im Sinne des Art. 4 Nr. 8 DSGVO, haben die Versicherten ihr gegenüber keinen Auskunftsanspruch aus Art. 15 DSGVO. Gemäß Art. 28 Abs. 3 lit. d) DSGVO ist der Auftragsverarbeiter jedoch verpflichtet, den Verantwortlichen bei der Erteilung der Auskunft zu unterstützen. Adressiert der Betroffene beispielsweise seinen Antrag versehentlich an die externe Stelle, so sollte ihn diese an die zuständige Krankenkasse weiterleiten.

\subsubsection{Externe Stelle als Verantwortlicher}

Erhält die externe Stelle die Sozialdaten im Rahmen einer Übermittlung dergestalt, dass sie eigenständig Verantwortlicher ist, besteht hier zusätzlich zu

244 Vgl. $\$ 83$ Abs. 1 S. 3 SGB X a.F.

245 Diering/Seidel, in: Diering/Timme, SGB X, 4. Aufl., 2016, §83 Rn. 4.

246 Heckmann/Paschke, in: Ehmann/Selmayr, DS-GV0, 2017, Art. 12 Rn. 43. 
dem Auskunftsanspruch gegenüber der gesetzlichen Krankenkasse auch ein Anspruch gegenüber der externen Stelle.

Für diesen Auskunftsanspruch gilt - sofern man, wie oben ausgeführt, von einer Anwendbarkeit der $\mathbb{6} 67 \mathrm{ff}$. SGB X ausgeht - dasselbe wie für den Auskunftsanspruch gegenüber der gesetzlichen Krankenkasse. Insofern kann vollumfänglich auf die obigen Ausführungen verwiesen werden.

Geht man hingegen davon aus, dass für den Auskunftsanspruch gegenüber der externen Stelle nur die allgemeinen datenschutzrechtlichen Anforderungen gelten, ergibt sich Folgendes:

- Die Einschränkung der $₫ 83$ Abs. 2 S. 1 entfällt. Die externe Stelle kann das Auskunftsersuchen daher nicht allein deswegen zurückweisen, weil es zu pauschal formuliert wurde. Die Auskunft kann jedoch gemäß $\mathbb{3} 34$ Abs. 4 BDSG - entsprechend $₫ 83$ Abs. 2 S. 2 SGB X - verweigert werden, wenn Angaben, die ein Auffinden der Daten ermöglichen, fehlen.

- Die Ausschlussgründe des $\mathbb{5} 83$ Abs. 1 SGB X entfallen, jedoch ergeben sich aus $\mathbb{3} 4 \mathrm{Abs}$. 1 BDSG entsprechende Verweigerungsrechte.

- Werden personenbezogene Daten ohne Einwilligung des Betroffenen für Forschungszwecke oder statistische Zwecke gemäß $\$ 27$ Abs. 1 BDSG erhoben, kann der Auskunftsanspruch zudem gemäß $\mathbb{2 7}$ Abs. 2 BDSG ausgeschlossen oder beschränkt werden. Dies ist der Fall, wenn andernfalls die Verwirklichung von Statistik- und Forschungszwecken unmöglich gemacht oder ernsthaft beeinträchtigt werden würde und der Ausschluss für die Erfüllung dieser Zwecke erforderlich ist. Die Verwirklichung des Forschungszwecks ist z.B. ernsthaft beeinträchtigt, wenn die zuständige Ethikkommission zum Schutz der betroffenen Person eine Durchführung des Projekts untersagen würde. ${ }^{247}$

- Der Auskunftsanspruch kann darüber hinaus ausgeschlossen werden, wenn die Daten für wissenschaftliche Forschungszwecke erforderlich sind und die Auskunftserteilung einen unverhältnismäßigen Aufwand erfordern würde. Dies kommt bereits dann in Betracht, wenn ein Forschungsvorhaben mit besonders großen Datenmengen arbeitet. ${ }^{248} \mathrm{Aller}-$ dings wird ein vollständiger Ausschluss des Auskunftsanspruchs auch hier wohl nur ausnahmsweise möglich sein. Analog zu den obigen Ausführungen kann der Aufwand der Auskunftserteilung beispielsweise dadurch verringert werden, dass der Betroffene sein Auskunftsersuchen auf bestimmte Informationen oder Verarbeitungsvorgänge beschränkt. ${ }^{249}$

- Außerdem kann die externe Stelle gemäß Art. 12 Abs. 5 DSGVO die Erteilung der Auskunft wegen offenkundiger Unbegründetheit oder exzessiver Antragsstellung verweigern. 
- Die Gründe für die Auskunftsverweigerung sind auch hier zu dokumentieren und dem Betroffenen mitzuteilen ( $\$ 34$ Abs. 2 BDSG).

- Ansonsten ergeben sich für den Auskunftsanspruch des Versicherten nach allgemeinem Datenschutzrecht keine Besonderheiten. Es gelten die obigen Ausführungen. 